\title{
The Structural Basis for Microtubule Binding and Release by Dynein
}

\section{Citation}

Redwine, William Bret. 2012. The Structural Basis for Microtubule Binding and Release by Dynein. Doctoral dissertation, Harvard University.

\section{Permanent link}

http://nrs.harvard.edu/urn-3:HUL.InstRepos:10436239

\section{Terms of Use}

This article was downloaded from Harvard University's DASH repository, and is made available under the terms and conditions applicable to Other Posted Material, as set forth at http:// nrs.harvard.edu/urn-3:HUL.InstRepos:dash.current.terms-of-use\#LAA

\section{Share Your Story}

The Harvard community has made this article openly available.

Please share how this access benefits you. Submit a story.

\section{Accessibility}


(C)2012 by William Bret Redwine 


\title{
The Structural Basis for Microtubule Binding and Release by Dynein
}

\begin{abstract}
Eukaryotic cells face a considerable challenge organizing a complicated interior with spatial and temporal precision. They do so, in part, through the deployment of the microtubulebased molecular motors kinesin and dynein, which translate chemo-mechanical force production into the movement of diverse cargo. Many aspects of kinesin's motility mechanism are now known in detail, whereas fundamental aspects of dynein's motility mechanism remain unclear. An important unresolved question is how dynein couples rounds of ATP binding and hydrolysis to changes in affinity for its track, a requisite for a protein that takes steps. Here we report a subnanometer cryo-EM reconstruction of the high affinity state of dynein's microtubule binding domain in complex with the microtubule. Using molecular dynamics flexible fitting, we determined a pseudoatomic model of the high affinity state. When compared to previously reported crystal structure of the free microtubule binding domain, our model revealed the conformational changes underlying changes in affinity. Surprisingly, our simulations suggested that specific residues within the microtubule binding domain may tune dynein's affinity for the microtubule. We confirmed this observation by directly measuring dynein's motile properties using in vitro single molecule motility assays, which demonstrated that single point mutations of
\end{abstract}


these residues dramatically enhance dynein's processivity. We then sought to understand why dynein has been selected to be a restrained motor, and found that dynein-driven nuclear oscillations in budding yeast are defective in the context of highly processive mutants. Together, these results provide a mechanism for the coupling of ATPase activity to microtubule binding and release by dynein, and the degree to which evolution has fine-tuned this mechanism. I conclude with a roadmap of future approaches to gain further insight into dynein's motility mechanism, and describe our work developing materials and methods towards this goal. 


\section{Table of Contents}

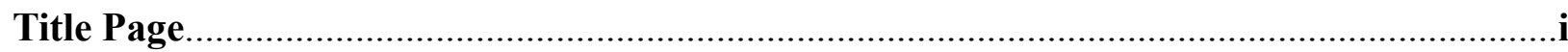

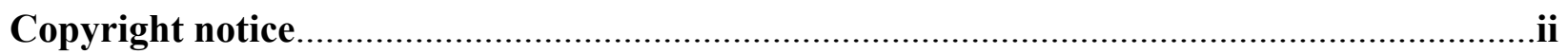

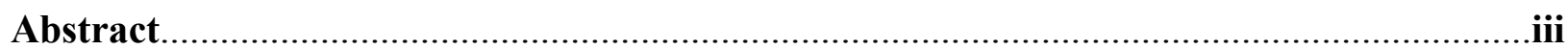

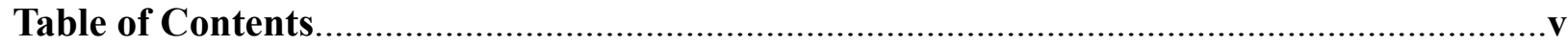

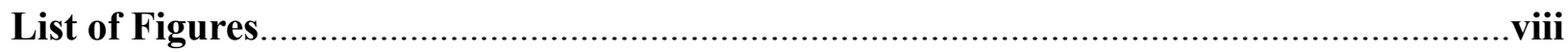

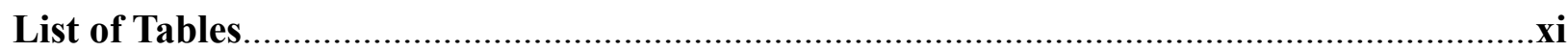

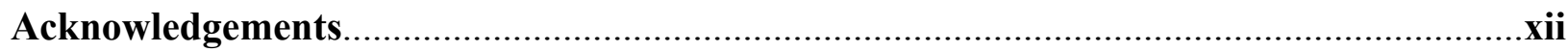

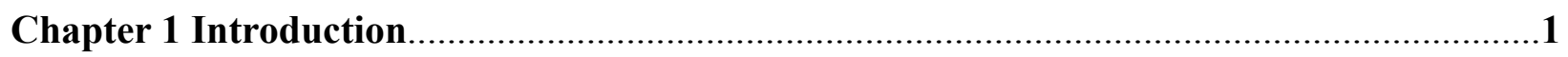

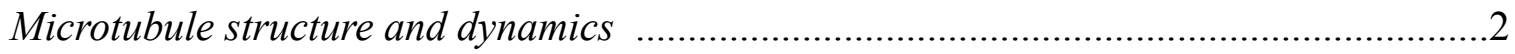

Microtubule-based molecular motors: compare and contrast........................................

Dynein structure and mechanistic implications.......................................................5

Dynein intramolecular communication: the helix sliding hypothesis..............................8

Recent advances in cryo-EM reconstructions of microtubule-motor complexes............... 12

Summary of approaches and results reported in this study....................................... 18

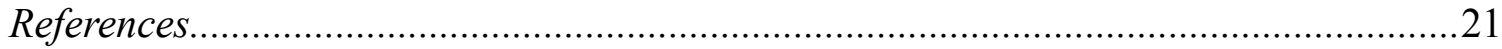

Chapter 2 Structural basis for microtubule binding and release by dynein......................28

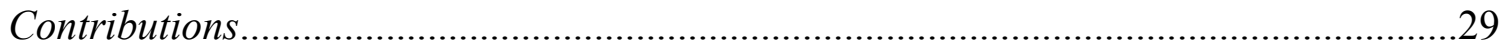

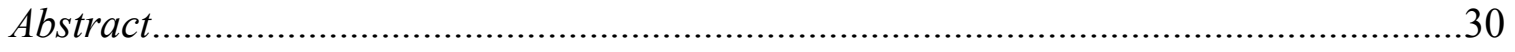

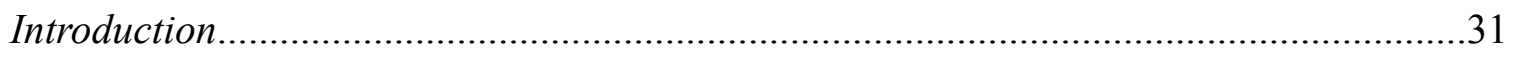

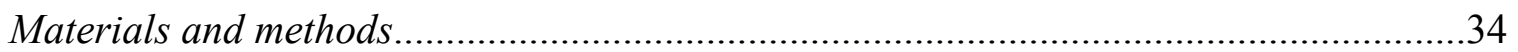


Results.

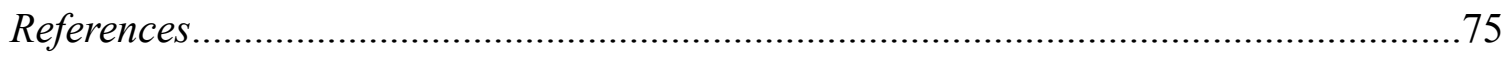

Chapter 3 Consequences of enhanced dynein processivity in vivo.......................................81

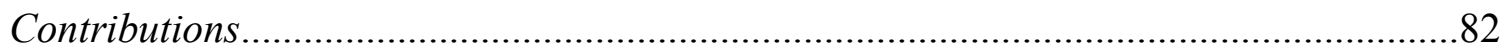

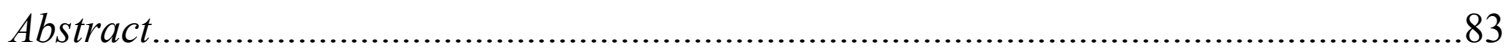

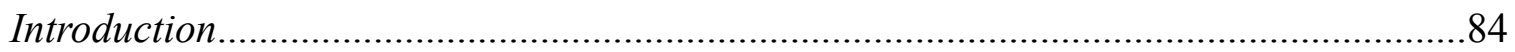

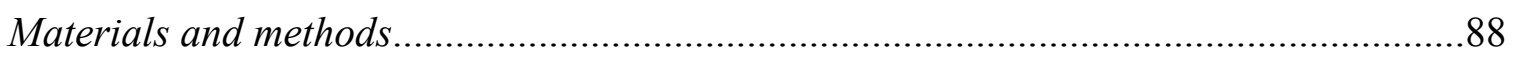

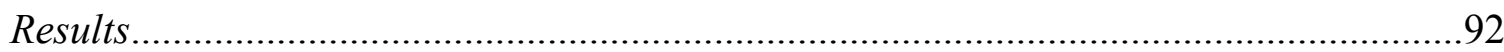

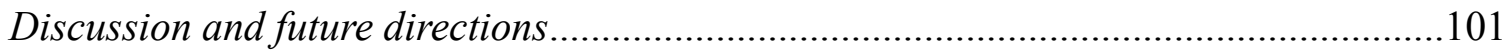

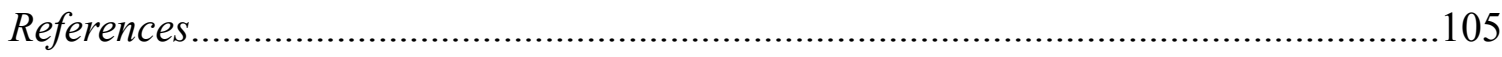

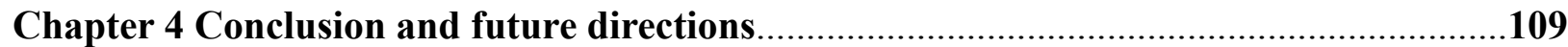

Appendix 1 Preparation and data collection of a minimal MTBD from Saccharomyces

cerevisiae

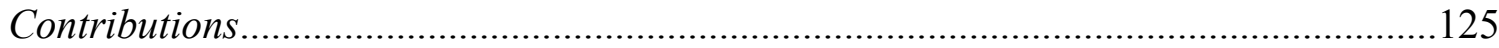

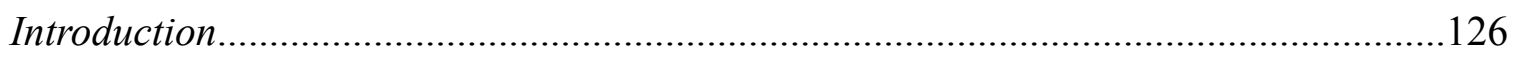

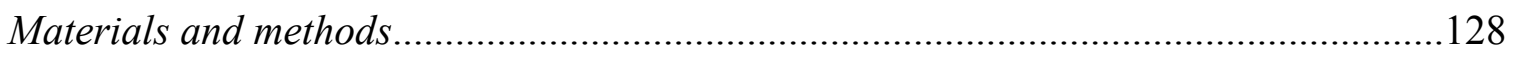

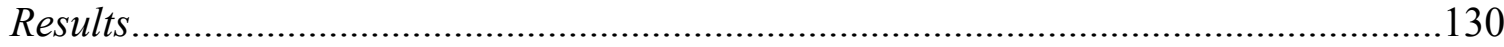

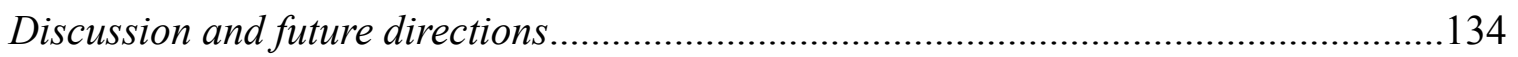

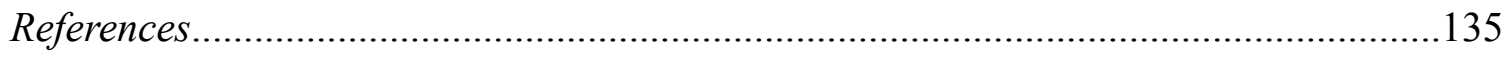

Appendix 2 Preparation of cytoplasmic dynein for cryo-electron microscopy....................136

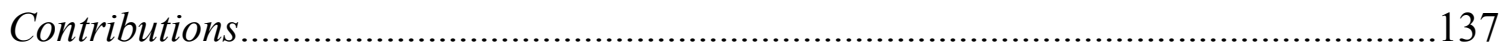

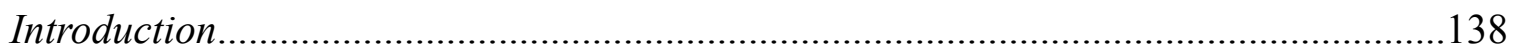




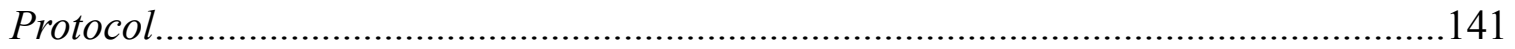

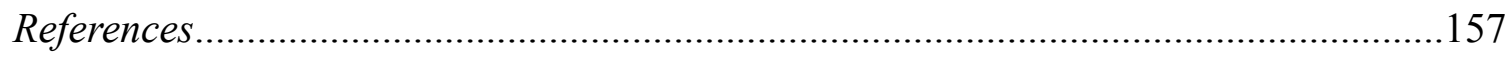




\section{List of Figures}

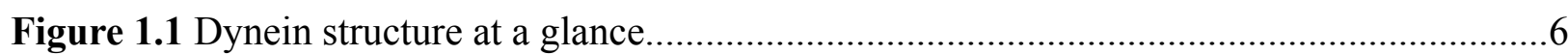

Figure 1.2 Key aspects of dynein's chemo-mechanical cycle..................................................

Figure 1.3 Comparison of microtubule binding by dynein and kinesin.......................................

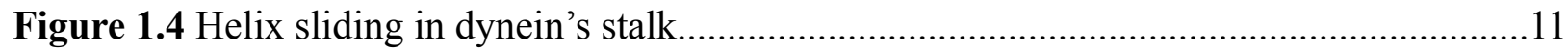

Figure 1.5. Atomic resolution structures of the free MTBD..................................................13

Figure 1.6 Helical and non-helical paths along the microtubule lattice......................................15

Figure 1.7 Single-Particle reconstruction of MT bound proteins..............................................17

Figure 2.1 Towards a structural understanding of the helix sliding hypothesis...........................32

Figure 2.2 Sub-nanometer MTBD-MT reconstruction...........................................................49

Figure 2.3 Complete reconstruction of the high-affinity SRS-MTBD bound to a MT.................50

Figure 2.4 The high-affinity, MT-bound state of the dynein MTBD is characterized by

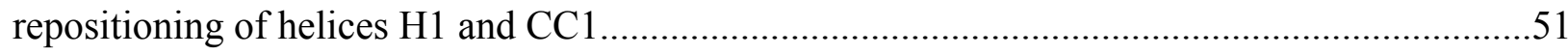

Figure 2.5 Molecular Dynamics study of the dynein MTBD conformations bound to MTs........52

Figure 2.6 Comparison of $10 \AA$ synthetic maps of the low- and high-affinity MTBD

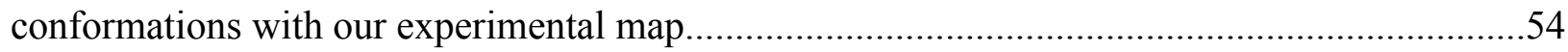

Figure 2.7 Different atomic resolution structures of the MTBD converge on a single MT-bound

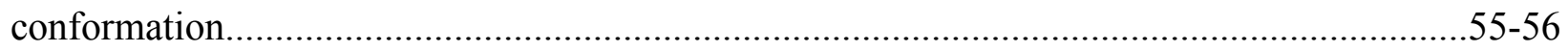

Figure 2.8 Conserved intermolecular MTBD-MT and intramolecular MTBD interactions in the

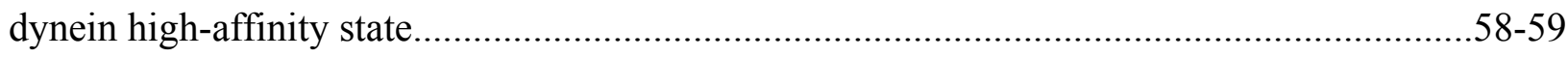


Figure 2.9 Formation of hydrogen bonds between the dynein MTBD and tubulin during MD

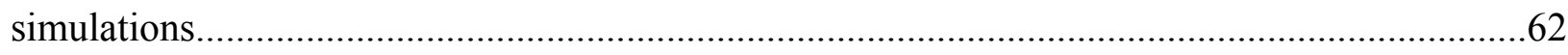

Figure 2.10 Effect of increasing ionic strength on dynein motility......................................63

Figure 2.11 Behavior of dynamic salt bridges in the MTBD as determined by MD.................64

Figure 2.12 Dynamic salt bridges in $\mathrm{H} 1$ and $\mathrm{H} 6$ of the MTBD as observed by MD

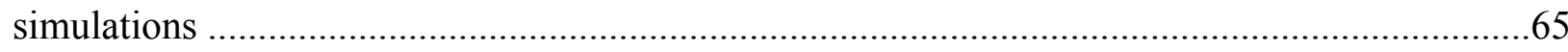

Figure 2.13 Dynamic salt bridges reduce dynein motility...............................................67

Figure 2.14 Dynamic salt bridges temper dynein motility...............................................68-69

Figure 2.15 Dynamic salt bridge mutations increase dynein processivity under more stringent

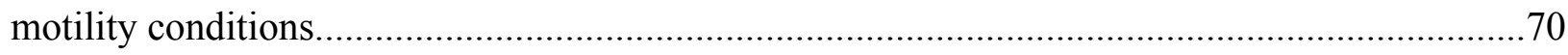

Figure 2.16 Dynamic salt bridge mutations increase dynein processivity independent of tubulin

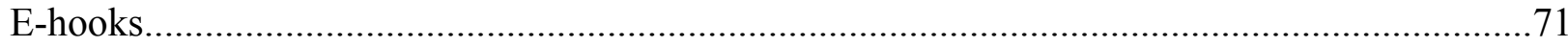

Figure 2.17 Intraflagellar transport dynein (cytoplasmic dynein 2) lacks a key intramolecular salt

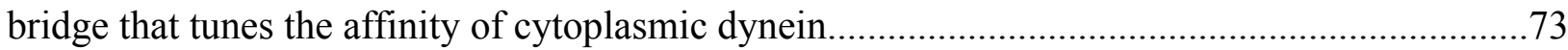

Figure 3.1 Two pathways contribute to maintenance of the mitotic spindle at the bud neck.......87

Figure 3.2 Imaging dynein-driven spindle movements in vivo........................................93

Figure 3.3 Automated tracking of spindle pole bodies in vivo............................................94

Figure 3.4 Symmetrically localized dynein in HU-arrested cells......................................95

Figure 3.5 Highly processive dynein mutations reduce the velocity of mitotic spindle movement in vivo 97

Figure 3.6 The effect of highly processive dynein mutations of mitotic spindle bud neck crossings in vivo .98 
Figure 3.7 The effect of highly processive dynein mutations of mitotic spindle localization in vivo 100

Figure 4.1 Uncoupling the head-MTBD bidirectional communication pathway 113

Figure 4.2 Determining if the MTBD is a self-contained, reversible microtubule sensor...........116

Figure A1.1 Purification of a minimal S. cerevisiae MTBD.....................................................131

Figure A1.2 Microtubules highly decorated with a minimal MTBD.......................................132

Figure A1.3 High quality ScMTBD data collection on a Titan Krios........................................133

Figure A2.1 Response of yeast harboring Gal-inducible dynein minidimer to galactose following

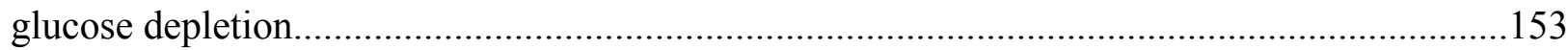

Figure A2.2 Large scale purification of dynein minidimer....................................................154

Figure A2.3 Gel filtration chromatography of IgG-agarose affinity purified ZZ-tagged dynein minidimer. 155

Figure A2.4 Highly decorated microtubules imaged under cryogenic conditions......................156 


\section{List of Tables}

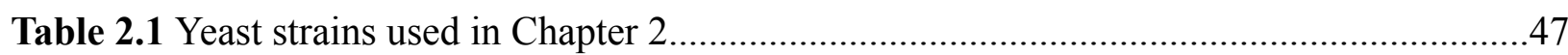

Table 2.2 Prominent interactions involving the high-affinity state of dynein's MTBD..............60

Table 2.3 Prominent interactions involving the low-affinity state of dynein's MTBD...............61

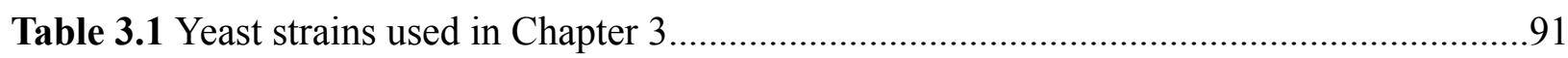




\section{Acknowledgements}

Now that my graduate studies are nearing their end, I feel as if I simply rode a wave of enthusiasm and support. I feel lucky to be able to leave content and proud to have had the opportunity to be part of exciting scientific research. If I had known it would be so much fun, I would have relaxed a bit more! I want to thank everyone for helping me achieve this goal.

I want to thank my previous mentors for encouraging me to consider a career in science. Gretchen Hagen at the University of Missouri first suggested I step away from dish washing to try my hand at research. She continues to cheer me on today. Joan and Ron Conaway at the Stowers Institute for Biomedical Research looked at my initial list of potential graduate schools and said, "aim higher.” I thank each of them for their encouragement, and for seeing what I often have difficulty seeing, my own potential.

I benefited from great groups of people in two separate labs. To all the members of both of my labs, I would like to thank them for providing a both rigorous scientific environment, as well as a fun and supportive place to work. In both of my labs I was fortunate to work as part of a team. Working with others towards a common goal taught me to put my ego aside and focus on the science. Seeing the speed with which we could answer tough questions taught me that collaboration is truly the way forward.

I must give special recognition to Rogelio Hernandez-Lopez, who has been a wonderful collaborator. I often joked that if we could combine our skills, we would be the perfect scientist. Such was the degree to which our interests and talents dovetailed. Rogelio also brings a level of sheer joy to science, the contribution of which to my success cannot be understated. 
The Biological and Biomedical Sciences (BBS) program at Harvard Medical School would simply no work if it were not for dedicated faculty and staff. The staff in the BBS office (Kate Hodgins, Maria Bollinger, Steve Obuchowski and Daniel Gonzalez) made navigating graduate school as painless as possible, and were always there for an encouraging discussion. Faculty like Tim Mitchison, Briana Burton, who served as the members of my advisory committee, were continual sources of critical feedback and support. I would like to thank the faculty members of my defense committee, Rachelle Gaudet, Niko Grigorieff, and Elizabeth Engle for sharing in this important part of my graduate studies. I would especially like to thank Fred Winston, a member of both committees, for his constant unbiased advice and support.

Working in two labs means having two bosses. For some graduate students that might constitute a cruel cosmic joke, but I consider myself fortunate to have Andres Leschziner and Samara Reck-Peterson as my mentors. I came to them at a difficult point in my graduate studies, and expected they would be skeptical of a fourth year student in search of a new scientific home. Their response was to accept me without reservation. The have tolerated my ups and downs, the waning and waxing of my enthusiasm. They always put people first, and I am indebted to them for their generosity, support, and continual enthusiasm.

Although I have never given them a practical reason to care for my work, my family has always shown interest and is a source of support I could not do without. My mother, Anne Redwine, and father, Bob Redwine, have transitioned into two of my best friends. My sister, Jill Pires, embarrasses me by constantly telling everyone their brother goes to Harvard, and my brother, Kirk Redwine, has been a great friend and constant presence, despite the distance. My 
new family, the members of the Cossor family, have embraced me as one of their own. I thank you all.

Lastly, I want to thank my biggest cheerleader, my wife. Furha accepts me without conditions, and supports me in everything I do. She is my best friend, and I love her and thank her with all my heart. 
Chapter 1 Introduction 


\section{Microtubule structure and dynamics}

Eukaryotic cells of all varieties must organize a complicated interior landscape with the timely sorting and delivery of a diverse set of materials. From individual mRNAs destined for localized translation, to the movement of entire nuclei in cells as different as migrating neurons and dividing yeast cells, the variety of packages that are transported is astounding. In part, cells have dealt with this challenge through the employment of a cytoskeletal network of actin filaments and microtubules. Along these roadways walk protein molecular motors that utilize the energy of ATP binding and hydrolysis to move diverse cargo or, when anchored, to pull their own roadways throughout the cell. In this introduction I focus on microtubules and the associated molecular motor dynein. I will include a special emphasis on dynein's molecular mechanism, and some of the tools used to elucidate its particulars. Finally, I conclude with an approach, the results of which are presented in subsequent chapters, to gain new insight into how dynein steps along microtubules.

Microtubules, which are self-assembling 2D crystals composed of alpha $(\alpha)$ and beta $(\beta)$ tubulin (reviewed in (1)), are used within cells to power many important processes. Free tubulin exists as a heterodimer of $\alpha \beta$-tubulin that can bind two molecules of GTP. Tubulin dimers interact longitudinally to form protofilaments, and laterally to form the tube structure that defines microtubules. One GTP, situated between $\alpha$ - and $\beta$-tubulin, is non-exchangeable and retains its triphosphate moiety; the other nucleotide is bound by $\beta$-tubulin, is solvent exposed and exchangeable. Addition of a new tubulin dimer stimulates the hydrolysis of the $\beta$-tubulin bound GTP to GDP; in this way, tubulin serves as its own GTPase activating protein. Comparison of the structures of unpolymerized tubulin (stathmin-like domain bound (2) or gamma tubulin (3)) 
with polymerized tubulin (zinc sheets (4), and models based on cryo-EM reconstructions (5)), supports a model whereby curved free tubulin dimers become straightened upon incorporation into the microtubule lattice (3). It should be noted that another model proposes that it is the GDP -> GTP exchange at $\beta$-tubulin itself that drives the curved to straight transition (6). Inherently strained by incorporation into the lattice and prone to disassembly, the ends of microtubules are not in equilibrium. Rather, they undergo transitions between prolonged growth and rapid shrinkage (catastrophe) (7). This is especially true for plus ends within cells, which are not stabilized as minus ends are by the gamma-TURC complex (8). The propensity to rapidly interconvert between growth and shrinkage, which is known as dynamic instability (7), is used by cells to define their shape $(9,10)$, search out cell cortices $(11)$, and to power the movement of large structures such as chromosomes $(12,13)$.

\section{Microtubule-based molecular motors: compare and contrast}

Cells also use microtubules as roadways, along which cargo are shuttled. This is accomplished by deploying microtubule-based molecular motors that walk along the microtubule via the coordinated stepping of motor domains (14). The two classes of microtubule-based molecular motors are kinesin and dynein. When attached to cargo, they are capable of transporting diverse materials throughout the cell, including mRNA, vesicles, and foreign cargo such as viruses (14). When motors are anchored to another structure via the end opposite their track-binding domains, they are capable of moving microtubules themselves. This second mode is dramatically employed by yeast, where tail-immobilized dynein pulls on microtubules to move an entire nucleus (15). Both classes of motors typically exists as dimeric assemblies of force- 
generating motor domains connected to dimerization and cargo-binding domains. They also both utilize ATP binding and hydrolysis to power their movement, bind to a common site in the tubulin intradimer interface (16), and are capable of processive "runs" consisting of many steps along the microtubule $(17,18)$.

In spite of some important similarities, kinesin and dynein are very different motors. Kinesin is more related to the actin-based motor myosin, and has sequence similarity to G proteins (19). On the other hand, dynein is a member of the AAA+ ATPase family (20). Most kinesins move processively towards the plus end of microtubules in discrete steps of $8 \mathrm{~nm}(18)$, corresponding to the distance between intradimer interfaces along a protofilament $(21,22)$. Its distribution of steps is tightly centered on $8 \mathrm{~nm}$, and backwards steps are rare. Kinesin takes steps along a single protofilament, as two-dimensional analysis of kinesin stepping shows a very small off-axis component (17). While dynein also takes many $8 \mathrm{~nm}$ steps, its step size distribution is wide, with many long steps that bypass the equivalent of 3-4 binding sites (17). Dynein takes backward steps with considerable frequency, and in some observed runs does so processively. The flexibility of its motility mechanism extends to off-axis steps as well, with side steps of several protofilaments being not uncommon (17).

Dynein's variable stepping behavior is likely tension-based. When its motor domains are close together, dynein exhibits stochastic stepping behavior, with an equal probability that either the leading or lagging head will take a step. However, as the head-head distance, and therefore the head-head tension, increases, the probability that dynein will take alternating steps increases $(23,24)$. Interestingly, heads often maintain a leading or lagging identity for many steps $(23$, 24). This stands in contrast to kinesin, which uses so-called "hand-over-hand" stepping, in which 
each head alternates between leading and lagging identities (25). Dynein's unique stepping behavior suggests that many structural aspects of its motility mechanism will be quite different from that of kinesin.

Dynein structure and mechanistic implications

Dynein uses a very unique domain architecture to couple nucleotide binding and hydrolysis to force production (Figure 1.1). Most AAA+ ATPase proteins, the family of which dynein is a member, self-assemble into hexameric rings from monomeric gene products (26). Electron microscopy (EM) studies of axonemal and cytoplasmic dynein first showed that dynein does share the common AAA+ feature of a hexameric ATPase ring, but, in an unusual variation, the entire ring is expressed as a single polypeptide $(27,28)$. Four of the ATPase domains, AAA1-4, are capable of binding nucleotide, with AAA1 being the primary site of hydrolysis (29-31). AAA2 cannot hydrolyze ATP, but plays a role in stabilizing a conformation of the powerstroke (32). AAA3 and AAA4 bind and hydrolyze ATP, and serve still unclear auxiliary roles (33). AAA5 and AAA6 lack consensus ATP binding and hydrolysis motifs, and most likely serve a structural role in completing the ring architecture $(34,35)$.

The linker domain is thought to couple ATP binding and hydrolysis to force production $(27,28,36)$. The linker is a long rod-like domain that drapes over the ring and undergoes large conformational changes in response to ATP binding and release $(27,28,36,37)$ (Figure 1.2). It is thought that these conformational changes constitute dynein's powerstroke, with beginning and ending states referred to as the pre- and post-powerstroke. The pre-powerstroke conformation has been visualized in two-dimensional EM studies, and is the predominant 


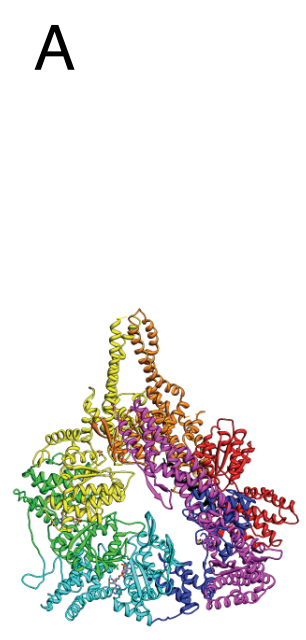

S. cerevisiae

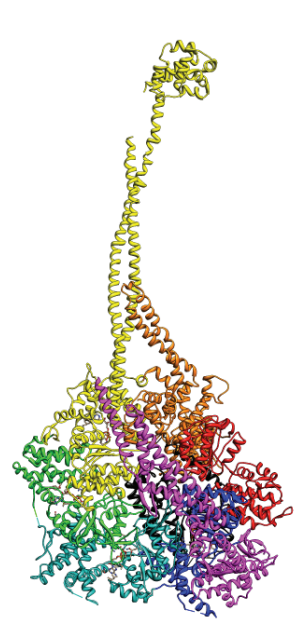

D. discoideum
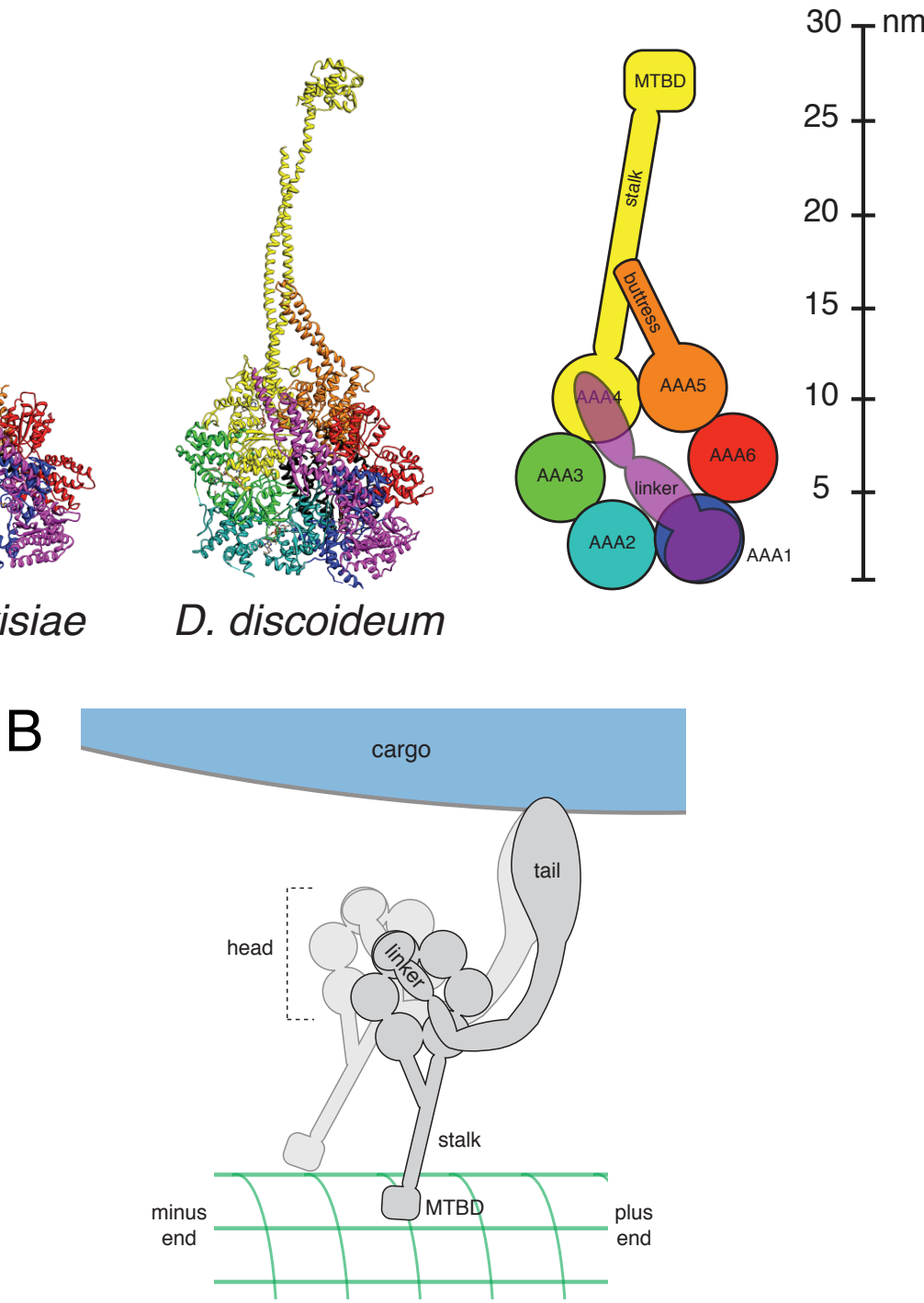

Figure 1.1. Dynein structure at a glance. (A) Comparison of $S$. cerevisiae (left) and $D$. discoideum (middle) dynein motor domain crystal structures. Color coding corresponds to the labeled structural elements in the cartoon (right). Note the linker domain is in the postpowerstroke conformation. (B) Cartoon depiction of a dynein dimer (grey) moving cargo (blue) along a microtubule (green lattice). Structural elements are labeled, including a representation of the tail domain based upon (56). Microtubule polarity is indicated. The cargo is drawn to scale as a $500 \mathrm{~nm}$ sphere. 


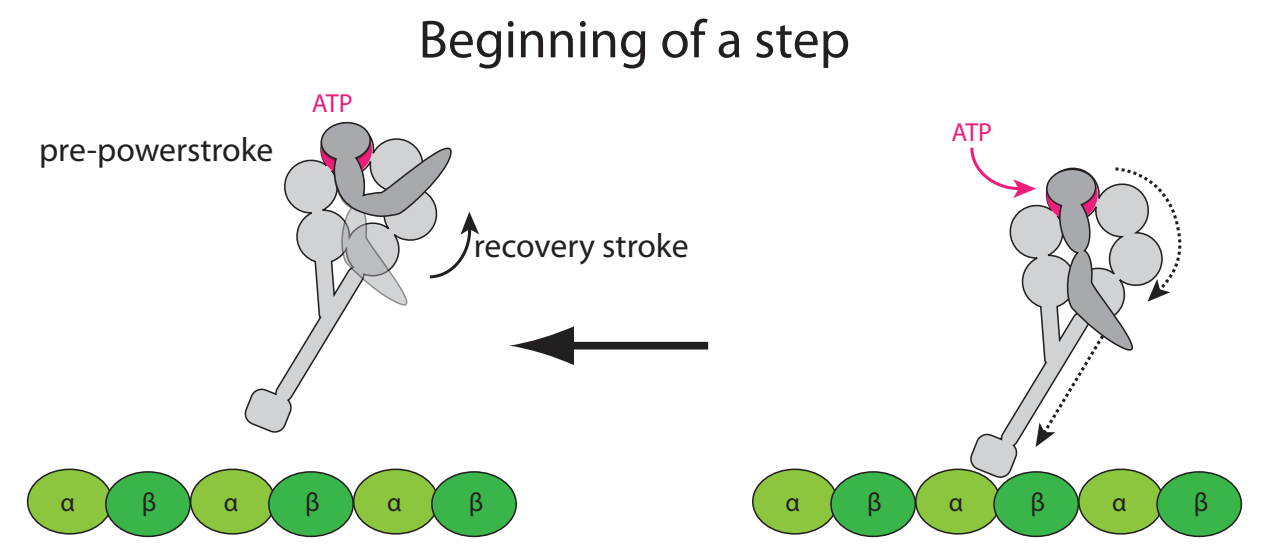

\section{End of a step}
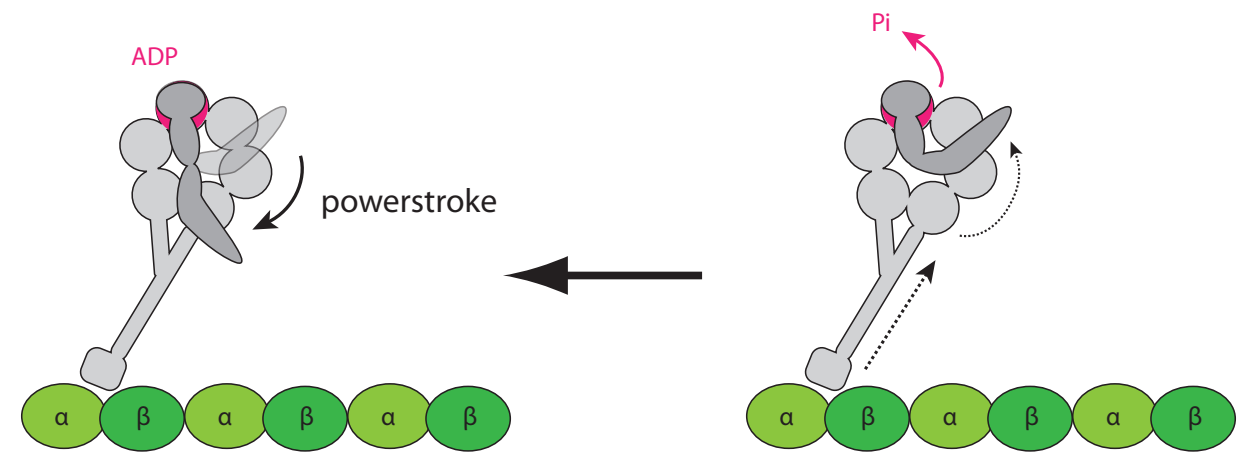

Figure 1.2. Key aspects of dynein's chemo-mechanical cycle. A dynein monomer (grey) is shown at the beginning (top) and end (bottom) of a step along a microtubule (protofilament shown, green). Refer to Figure 1.1 for details on domain architecture and a hypothetical arrangement of the heads relative to cargo. (Top) ATP binding induces a recovery stroke (small solid arrow) in the linker domain via intramolecular communication (dashed arrrows). (Bottom) Binding to the microtubule causes the release of phosphate and a subsequent powerstroke (small solid arrow) via intramolecular communication (dashed arrows). Based on data from $(28,36,37$, $42,55)$. 
conformation adopted under nucleotide states that exist before the release of phosphate (ATP, and $\mathrm{ADP}+\mathrm{Pi}$ or its mimic ADP + vanadate) $(27,28,37)$. The post-powerstroke conformation, a conformation seen in crystal structures $(32,34,35,38), 2 \mathrm{D}$ EM $(27,28)$, is adopted under ADP and no nucleotide conditions, and occurs upon the release of phosphate (32). In this conformation the linker spans the ring from AAA1 to form interactions between its $\mathrm{N}$-terminus and either AAA4 or $5(32,34,35,38)$. ATP binding resets the position of the $\mathrm{N}$-terminal portion of the linker from AAA4/5 to AAA2, constituting the recovery stroke $(36,37)$.

Dynein's domain architecture results in a mechanistically important arrangement - unlike kinesin, dynein does not have its ATPase and polymer track binding sites located within a single domain (Figure 1.3). The ATP state of the head is tightly connected to microtubule affinity (36) (Figure 1.2). Connecting the ATPase ring to the microtubule is the stalk, a long antiparallel coiled coil tipped by the microtubule binding domain (MTBD) $(39,40)$. The distance from kinesin's ATP site to the MT surface is only a couple of nanometers (41). Dynein's stalk is nearly $15 \mathrm{~nm}$ long from the point it exits from the ring (AAA4) to the tip of the globular MTBD $(27,32)$. If one adds to that the distance across the ring to AAA1, nearly $25 \mathrm{~nm}$ separates the main site of ATP hydrolysis and the MTBD. This very dramatic spatial separation implies that considerable intramolecular communication must occur to couple ATP binding and hydrolysis with the changes in microtubule affinity.

\section{Dynein intramolecular communication: the helix sliding hypothesis}

Currently, the best explanation as to how dynein couples ATP binding and hydrolysis to changes in the affinity of the MTBD for microtubules is the helix sliding hypothesis (Figure 1.4). 


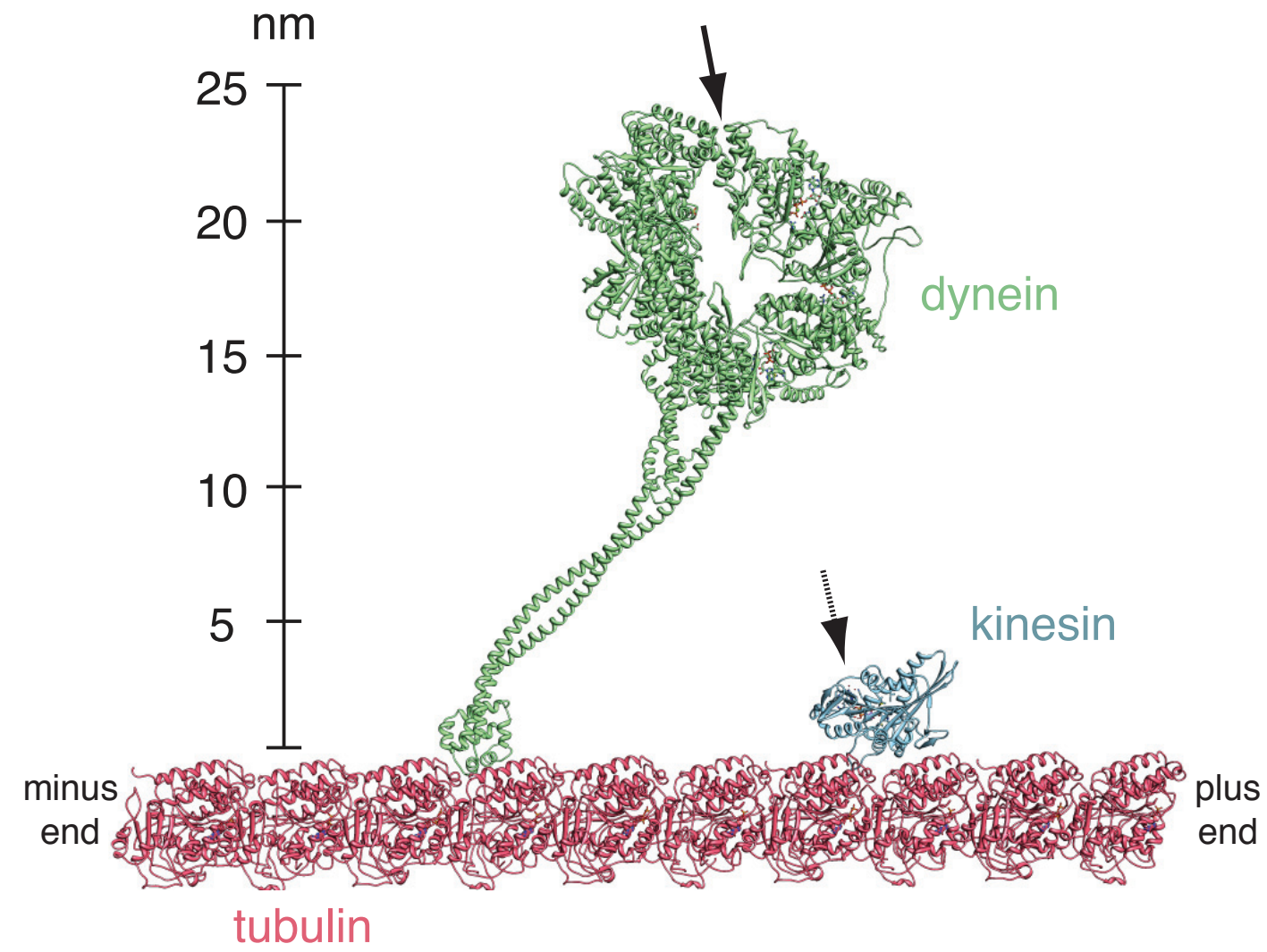

Figure 1.3. Comparison of microtubule binding by dynein and kinesin. A dynein monomer (green) modeled upon reported crystal structures (PDB 3QMZ (32), PDB 3ERR (55)) and bound to the microtubule (pink) according to a previous low resolution cryo-EM reconstruction (55). Kinesin (blue) binding is based upon a sub-nanometer cryo-EM reconstruction (40). Solid arrow points to the primary ATPase of dynein. Dashed arrow points the ATP binding pocket of kinesin. Scale bar indicates distance from the microtubule surface. Microtubule polarity is indicated. 
This hypothesis was first investigated in 2005 with a study initiated by Ian Gibbons (42), who first described dynein in 1965 (43). Alignments of the stalk and MTBD from various species revealed a divergence in dynein sequence from canonical antiparallel coiled coils. The alpha helices that comprise antiparallel coiled coils have as their repeated unit the heptad repeat, with positions typically lettered "a" through "g". While $\mathrm{CC} 2$, the half of the stalk that exits the MTBD and re-enters the head, contains conserved hydrophobic residues at positions "a" and "d", $\mathrm{CC} 1$ has an average of only 1 conserved hydrophobic residue per heptad repeat. This arrangement results that $\mathrm{CC} 1$ can adopt two possible registries relative to $\mathrm{CC} 2$ (termed $\alpha$ and $\beta$ ) that satisfy the packing at the core of the coiled-coil. Gibbons et al. (42) fused the stalk and MTBD of dynein to a truncated antiparallel coiled coil within seryl-tRNA synthetase (SRS), and systematically altered the length of $\mathrm{CC} 1$ sequence relative to $\mathrm{CC} 2$. Microtubule binding assays revealed that the differences in microtubule affinity between the $\alpha$ and $\beta$ registries was nearly 20 fold (42). These results suggested that bidirectional communication, linking the ATP state of the head to microtubule affinity, was mediated by helix sliding in the stalk.

The helix sliding hypothesis gained further traction with a study that explored how forcing specific stalk registries within an entire motor effects bidirectional communication (44). The authors created dynein constructs with cysteine pairs predicted to form disulfide bonds in specific stalk registries. They found that the stalk continuously explores a variety of registries, including the previously described $\alpha$ and $\beta$ registries (42). The preferred registry depends on the nucleotide state, and fixed stalk registries uncouple the motor from its microtubule sensing abilities (44). Typically, due to cycles of binding and release, a WT monomeric motor given sufficient ATP will exhibit a lower bulk affinity for microtubules than one deprived of ATP. 


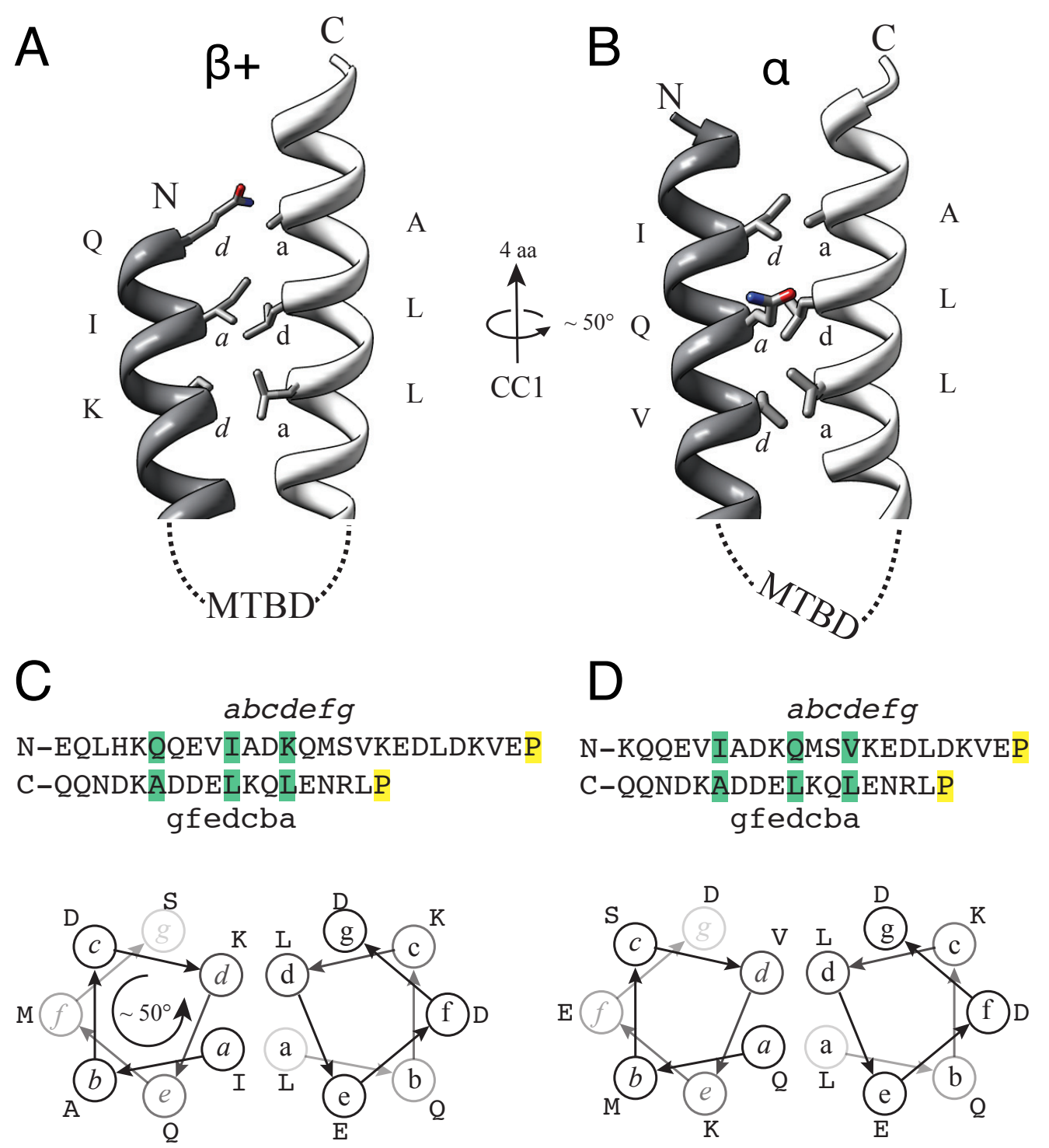

Figure 1.4. Helix sliding in dynein's stalk. (A) A section of the coiled coil from a low affinity, $\beta$-registry, SRS-MTBD construct (PDB 3ERR, (55)). $\mathrm{N}$ and $\mathrm{C}$ termini are indicated. Amino acids forming hydrophobic interactions are displayed and indicated with their corresponding one letter amino acid codes (outside) and heptad repeat letter code (inside). (B) A high affinity alpha registry form of the coiled coil was generated by applying a 4 amino acid upward shift and $\sim 50^{\circ}$ counterclockwise rotation. Note the movement of a labelled isoleucine (I), which moves from the " $a$ " position to the" $d$ " position in the preceding heptad repeat. (C) Top: alignment of the heptad repeats in (A). Hydrophobic interactions from (A) are colored green. Conserved proline residues are colored yellow as a reference point. A single heptad repeat is indicated for each helix. Bottom: helical wheel depiction of a single heptad repeat (top). Distance away from viewer is indicated with increasing transparency. (D) Equivalent to (C) and corresponding to the $\alpha$-registry displayed in (B). 
However, when a full length motor is forced into the high affinity $\alpha$ registry (42) it is insensitive to ATP, and binds to microtubules with high affinity (44). Furthermore, when locked in the $\alpha$ registry, the ATPase rate of this motor is insensitive to the presence of microtubules, remaining at maximal rates across microtubule concentrations (44). The opposite is true for the $\beta+$ registry, which is associated with low affinity for microtubules, and a low microtubule-stimulated ATPase rate (44).

Together the described studies provide compelling evidence that helix sliding is how dynein couples the state of its ATPase subunits to the microtubule affinity of its MTBD (see Figure 1.2). Therefore, in the context of stepping along microtubules, the MTBD is the beginning and end of signals needed to take a step. When dynein takes a step, it is the first component to "sense" the microtubule; when dynein needs to unbind at the start of a new step, it is what ultimately must respond to directions from the head. An important outstanding question, therefore, is what happens to the MTBD during microtubule affinity transitions. Does it undergo large conformation changes? How is it coupled to helix sliding? A complete understanding of how dynein alternates between high and low affinity requires structures of both the free low affinity MTBD as well as the high affinity state bound to microtubules. While structures of the free MTBD are available $(32,55)$, it is unclear what constitutes the high affinity conformation (Figure 1.5). Furthermore, there are no high resolution structures of the MTBD bound to microtubules to clarify this issue. As I will discuss below, microtubules, which are not amenable to x-ray crystallography, necessitate innovative hybrid approaches for structure determination.

Recent advances in cyro-EM reconstructions of microtubule-motor complexes 


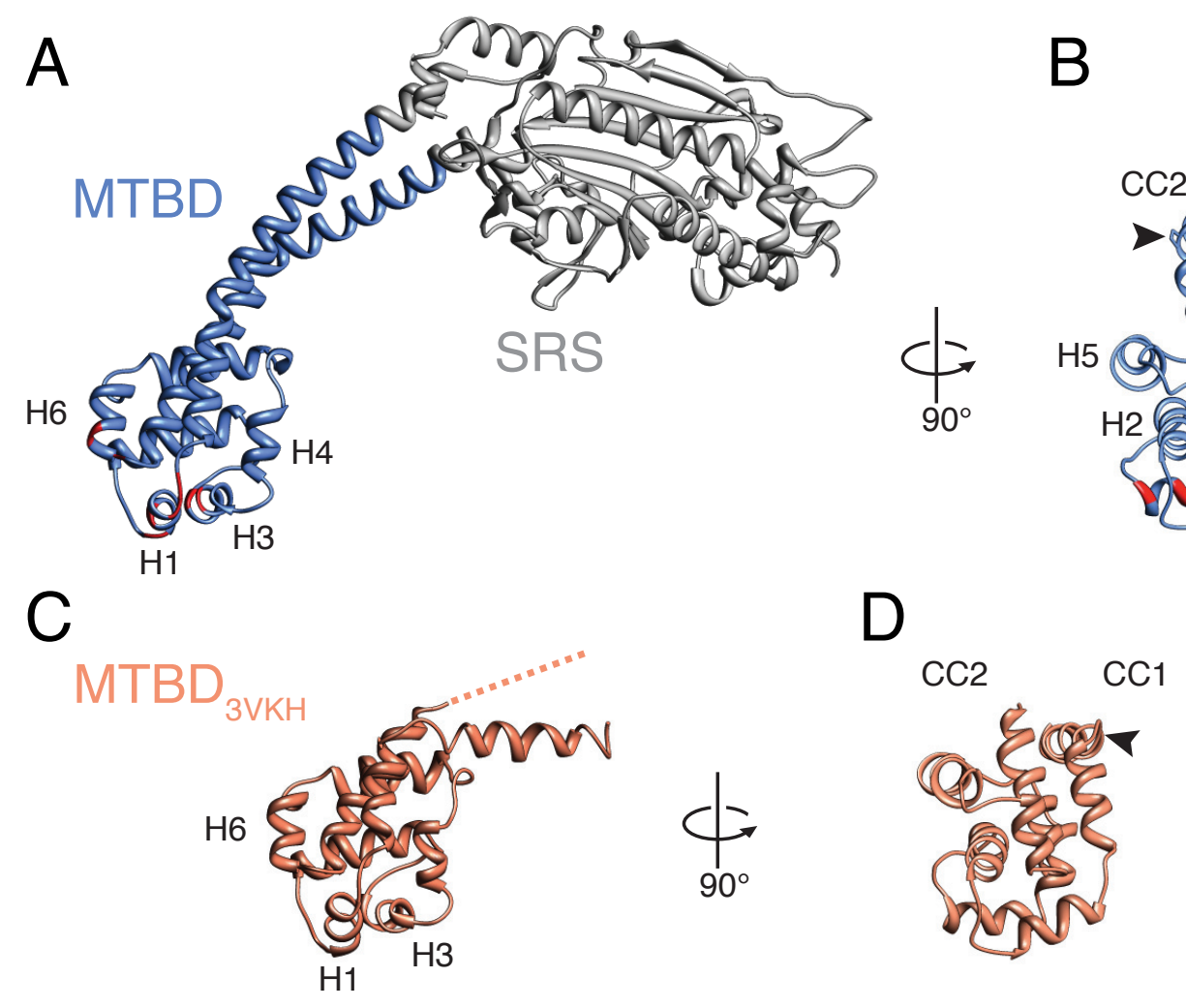

Figure 1.5. Atomic resolution structures of the free MTBD. (A, B) Ribbon diagram of the low affinity ( $\beta+$ registry) SRS-stalk-MTBD fusion (PDB 3ERR (55)). MTBD ands talk are colored blue, the SRS is colored grey. Residues previously reported to disrupt microtubule binding are colored red (62). Helices are in the MTBD are labelled. SRS has been omitted in (B) for clarity. Conserved prolines (arrowheads) create the kink in the stalk (comprised of helices $\mathrm{CC} 1$ and $\mathrm{CC} 2$ ). Note packing of the base of $\mathrm{CC} 2$ against $\mathrm{H} 2$ and $\mathrm{H} 5$; $\mathrm{CC} 1$ has fewer contacts with the rest of the MTBD. (C, D) Ribbon diagram of the MTBD portion from a monomeric dynein construct from $D$. discoideum (PDB $3 \mathrm{VKH}(32)$ ). Note the absence of $\mathrm{CC} 2$ in the portion of the stalk past the proline induced kink (indicated with dashed line). $3 \mathrm{VKH}$ was crystallized in a high affinity state of the ATPase-containing ring (ADP, (36)), yet it has a MTBD conformation nearly identical to that in the SRS-MTBD fusion. This raises the possibility that microtubules are needed to stabilize a conformationally distinct high affinity conformation of the MTBD. 
For many years the method of choice for microtubule structure determination involved cryo-EM data collection and the use of Fourier-Bessel helical reconstruction (45). This approach has proven a valuable tool for 3D reconstruction of samples with helical geometries; in fact, it is the technique that was used to generate the first EM-based 3D reconstruction (46). It has yielded numerous structures of microtubule-bound proteins, and was used extensively with notable success by the Milligan group (see (47) where they outline this approach and predict its contributions to microtubule motor protein research). The limitation with the Fourier-Bessel approach for MT structures calculated in this manner is that they never approached resolutions below $\sim 15 \AA$ (48). These structures provided valuable information about the sites on tubulin used by interacting proteins, and allowed envelope driven-docking of crystal structures. However, their limited resolution precluded flexible fitting of secondary structure elements (where resolutions of $<10 \AA$ are required), and the generation of accurate pseudoatomic models. As such, many molecular details of motor-microtubule interactions were simply not attainable.

One key to pushing reconstructions of microtubules to higher resolutions was the application of single-particle reconstruction techniques to quasi-helical microtubules. Only very rare microtubules, for example those containing 15 or 16 protofilaments, are truly helical, with continuous helices of homotypic lateral tubulin interactions. In vitro the vast majority of microtubules are quasi-helical, with a discontinuity known as the seam $(49,50)$ (Figure 1.6 A). The first sub-nanometer reconstruction of the microtubule (22) used projection matching of segments of microtubules from micrographs to a synthetic microtubule model. This study, which used data from 13-protofilament microtubules, disregarded the presence of the seam. Since $\alpha$ and $\beta$ - tubulin are essentially identical at resolutions lower than $6 \AA(51)$, one can ignore the 


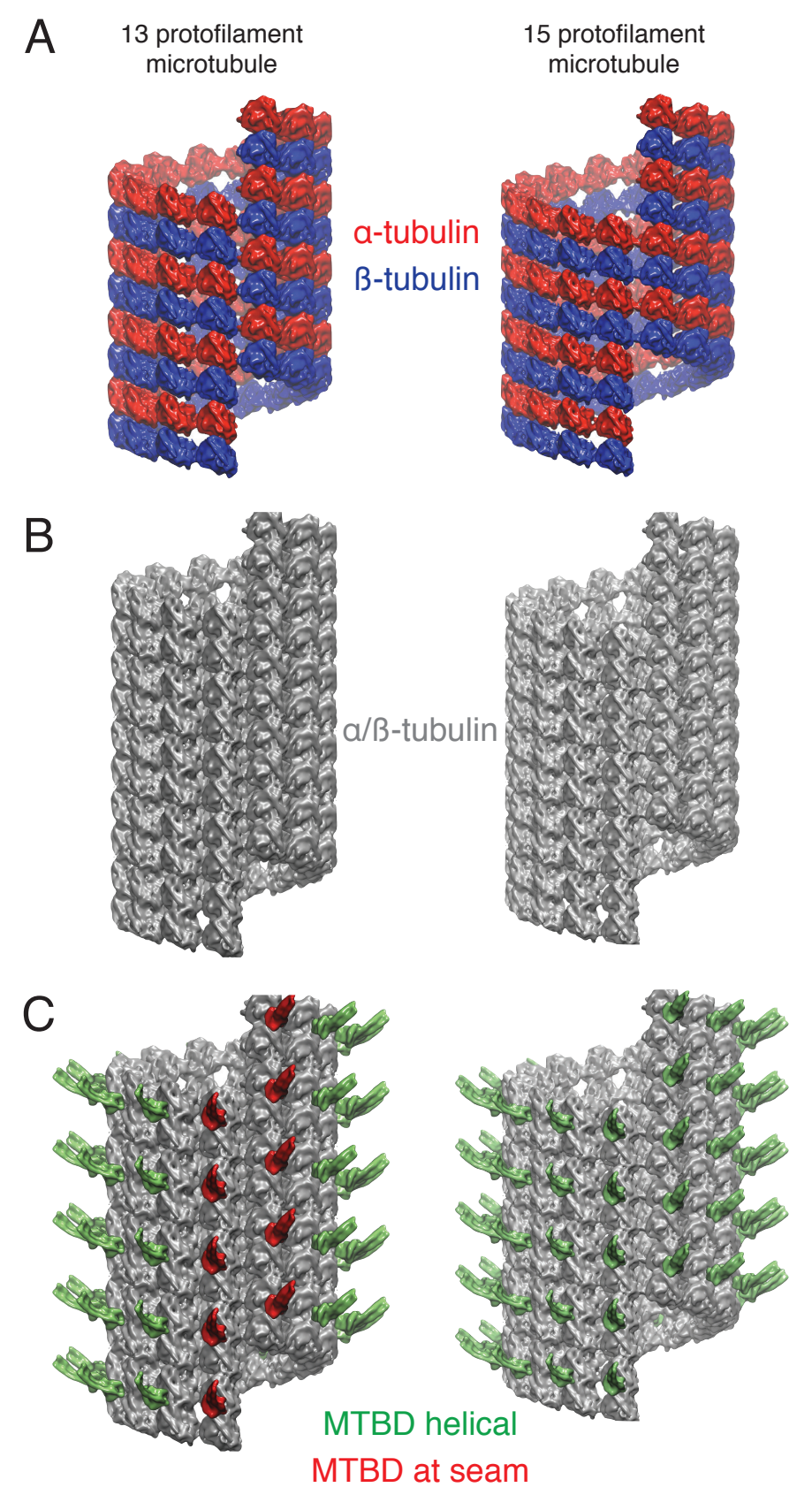

Figure 1.6. Helical and non-helical paths along the microtubule lattice. In each panel the left and right columns are $8 \AA$ renderings of 13 and 15 protofilament microtubules, respectively. (A) If one knows the identity of alpha and beta tubulin, 13 protofilament microtubules have a discontinuity known as the seam, whereas 15 protofilament microtubules are perfectly helical. (B) At resolutions currently obtainable by cryo-EM $(\sim 8 \AA)$, alpha and beta tubulin are identical, and both 13 protofilament microtubules can be treated as helical. (C) When decorated with a protein that binds every $8 \mathrm{~nm}$ (MTBD, green and red), the seam reappears due to a discontinuity in the bound protein's helical path. 
differences between the two and treat any microtubule as helical and seamless (22) (Figure 1.6 B). This fact was exploited recently to solve structures of microtubules with protofilament numbers ranging from 11 to 16 (52). Importantly, approaches that ignore the seam produce symmetrized volumes with a single tubulin monomer that is an average of $\alpha$ - and $\beta$ - tubulin.

In order to reconstruct volumes containing proteins bound to seamed microtubules, for example the kinesin motor domain and the MTBD of dynein, it is vital that one correctly identifies the seam (Figure 1.6 C). If a protein binds to tubulin at every monomer, as Ndc 80 has been shown to do (53), then the approach described above for undecorated microtubules is appropriate. Dynein and kinesin bind at the interval of the intradimer interface $(8 \mathrm{~nm})(16)$, the cleft formed along the outside of the microtubule where $\alpha$ - and $\beta$ - tubulin meet within a tubulin heterodimer. With such a binding pattern, only seamless microtubules will also contain continuous helices of the decorated protein (Figure 1.6 C). Since the vast majority of in vitro polymerized microtubules contain seams, and cryo-EM data collection is a time consuming process that encourages one to maximize the use of collected data, the seam must be accounted for.

The first sub-nanometer reconstruction of kinesin bound to microtubules presented a useful approach to single-particle reconstruction of decorated, seamed microtubules $(41,54)$ (Figure 1.7). After scanning collected micrographs, one digitally divides the microtubule into small segments. For 13 and 14 protofilament microtubules, where the supertwist (the degree to which protofilaments twist along the length of the microtubule) is non-existent or negligible, one can then subdivide and average the boxes into segments representing single tubulin dimer 


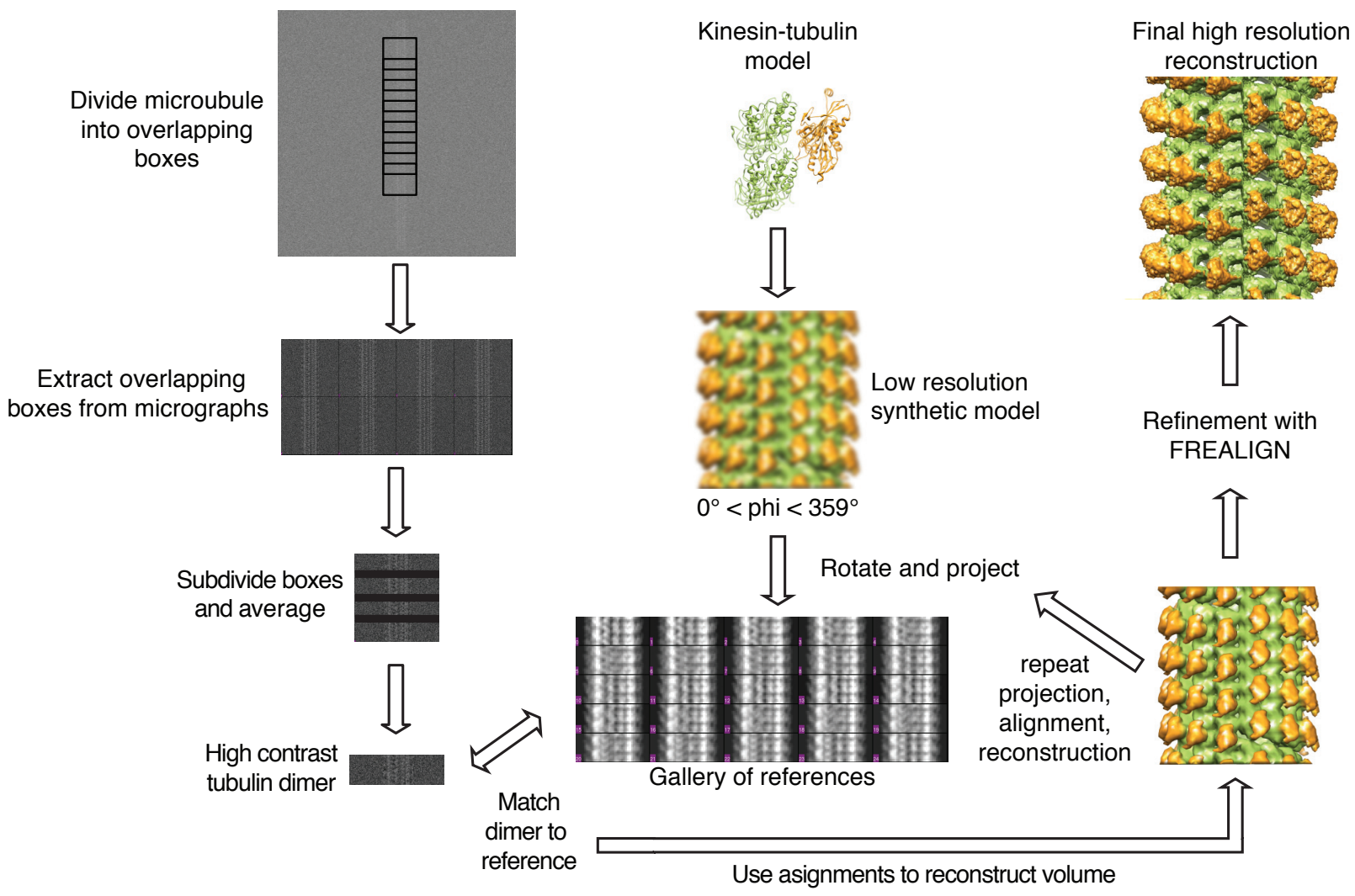

Figure 1.7. Single-particle reconstruction of MT bound proteins. In this example the kinesinmicrotubule interaction is used as reported (41). (Left column) Cryo-EM data is collected and digitized. Microtubules are selected and boxed into overlapping segments of $\sim 8-10$ tubulin dimers. These boxes are further subdivided into single tubulin dimers and averaged for increased contrast. (Middle column) A synthetic reconstruction is created with crystal structures. The volume is rotated (axially and out-of-plane) and projections calculated; these are the references. Dimer averages are then matched to the references (essential for identifying the seam), and the assigned angles are used to create a reconstruction by back projection. This process (alignment and reconstruction) can be repeated to convergence. (Right panel) In the final step, FREALIGN (61) is used to refine the reconstruction to higher resolution. Not shown is a step whereby the protofilaments are averaged and used to create a reconstruction containing 13 (or 14 in Chapter 2) copies of a single symmetrized protofilament. 
repeats. These averages are then matched against $2 \mathrm{D}$ projections of a simulated low resolution model of a microtubule decorated with the construct of interest. The seam in a 13 or 14 protofilament microtubule is nearly parallel to the microtubule axis; as such, as a quality control step, one determines how many segments from a given microtubule were assigned the same orientation of the seam. Alignment and quality assessment are repeated across hundreds of segments that represent many thousands of tubulin dimers, and a 3D volume is calculated by the back projection method. This method uses the angles and shifts assigned to each segment relative to the model microtubule to derive a single volume that incorporates all the data, dramatically increasing the signal to noise ratio. The signal amplification is further increased by a factor related to the protofilament number when the individual protofilaments are averaged to create a single composite protofilament containing all of the accepted data. In order to produce a microtubule volume, the protofilament is digitally replicated, rotated, and shifted according to the known symmetry of the microtubule.

The application of single-particle reconstruction techniques to microtubule bound proteins has contributed to important advances in our understanding of their mechanisms of action. For many of these proteins crystal structures have been solved, making them perfect candidates for hybrid approaches that apply flexible fitting and molecular dynamics. In this way pseudoatomic models that describe a protein's interaction with the microtubule lattice can be generated, interrogated, and ultimately tested.

Summary of approach and results reported in this study 
Cytoplasmic dynein is well suited for interrogation by cryo-EM and single-particle three dimensional (3D) reconstruction techniques. The high affinity state of the MTBD in complex with microtubules is known only at a resolution of $20-30 \AA(16,55)$, whereas an atomic resolution structure of the low affinity state has been reported (55). This provides an opportune moment to complete a structural picture of the helix sliding hypothesis. New structures of free monomeric dynein have been reported (56), with insights into linker conformation, but only low resolution structures are available for a microtubule bound version (57). As a result, many outstanding questions, including the angle of dynein's stalk and linker conformation, are not known for microtubule-bound monomeric dynein. Given that dynein is a force-generating enzyme $(58,59)$, it will be important to understand the arrangement of its force-generating elements and the vector along which these are applied. Complete reconstructions of the motor on microtubules would be invaluable towards this goal. The situation for dimeric dynein is even more open - there are no known 3D structures of motility-competent dimeric dynein, either free or microtubule bound, currently available. Considerable detail about dynein's motility mechanism has been reported $(17,23,24)$; understanding how it interacts with, and how its various mechanical elements are oriented with respect to, the microtubule will provide a much needed structural framework for these data.

In this thesis I present a hybrid approach used to uncover the structural basis for how the microtubule-based motor dynein alters its affinity for microtubules, enabling it to bind and release microtubules as it takes steps. In Chapter 2 we describe the application of single-particle reconstruction methods, previously applied to sub-nanometer reconstructions of kinesin bound to microtubules (41), to the 3D reconstruction of the high affinity conformation of dynein's MTBD 
bound to microtubules. The sub-nanometer resolution of this reconstruction allowed us to pursue molecular dynamics flexible fitting (60). We determined a pseudoatomic model of the high affinity conformation, which revealed the conformational changes that occur during transitions between low and high affinity. Detailed analysis of molecular dynamics simulations revealed dynamic salt bridges that alternate between MTBD-microtubule and intra-MTBD interactions. We reasoned that these salt bridges temper dyneins affinity for microtubules, which we confirmed by single molecule motility assays. We found that dynein processivity can be increased up to 6-fold when individual salt bridges are disrupted.

The salt bridges we report in Chapter 2 are conserved, and yeast could accumulate the mutations necessary for enhanced dynein processivity with single nucleotide changes. Why then is dynein restrained? In Chapter 3, we describe our efforts to understand why dynein has been selected for this apparent sub-maximal motility. We applied single molecule tracking to dyneindriven nuclear movements. We found significant defects in dynein-driven nuclear oscillations in live yeast when high processivity mutations were introduced, providing an indication why dynein is maintained with low basal processivity.

In Chapter 4, I conclude with an outline of future directions. This includes an emphasis on biochemical approaches to understanding intramolecular bidirectional communication, as well as efforts to reconstruct 3D EM volumes of more biochemically complicated dynein constructs bound to microtubules. Related to this, I include two appendices that describe methods developed towards these goals. The work encompassed in this thesis, as well completion of outlined future directions, will add towards a better understanding of the structural underpinnings of dynein's motility mechanism. 


\section{References}

1. E. Nogales. Structural insights into microtubule function. Journal of Cell Biology 67, 277-302 (2000).

2. R. B. G. Ravelli et al., Insight into tubulin regulation from a complex with colchicine and a stathmin-like domain, CORD Conference Proceedings 428, 198-202 (2004).

3. L. M. Rice, et al., The lattice as allosteric effector: Structural studies of $\alpha \beta$-and $\gamma$-tubulin clarify the role of GTP in microtubule assembly, Proc Natl Acad Sci USA 105, 5378 (2008).

4. E. Nogales et al., Structure of the alpha,beta-tubulin dimer by electron crystallography, Nature 391, 199 (1998).

5. D. B. Wells, A. Aksimentiev, Mechanical properties of a complete microtubule revealed through molecular dynamics simulation, Biophysical Journal 99, 629-637 (2010).

6. E. Nogales, H.-W. Wang, Structural mechanisms underlying nucleotide-dependent selfassembly of tubulin and its relatives, Curr Opin Struct Biol 16, 221-229 (2006).

7. T. Mitchison, M. Kirschner, Dynamic instability of microtubule growth, Nature 312, 237-242 (1984).

8. J. M. Kollman et al., Microtubule nucleating $\gamma$-TuSC assembles structures with 13-fold microtubule-like symmetry, J Cell Biol 466, 879-882 (2010). 
9. L. G. Tilney, K. R. Porter, Studies on the microtubules in heliozoa. II. The effect of low temperature on these structures in the formation and maintenance of the axopodia, J Cell Biol 34, $327-343$ (1967).

10. L. G. Tilney, J. R. Gibbins, Microtubules in the formation and development of the primary mesenchyme in Arbacia punctulata. II. An experimental analysis of their role in development and maintenance of cell shape, J Cell Biol 41, 227-250 (1969).

11. J. L. Carminati, Microtubules Orient the Mitotic Spindle in Yeast through Dynein-dependent Interactions with the Cell Cortex, J Cell Biol 138, 629-641 (1997).

12. A. Ashkin et al. a, Force generation of organelle transport measured in vivo by an infrared laser trap, Nature 348, 346-348 (1990).

13. S. Westermann et al., Structures and functions of yeast kinetochore complexes, Annu. Rev. Biochem. 76, 563-591 (2007).

14. R. D. Vale, The molecular motor toolbox for intracellular transport, Cell 112, 467-480 (2003).

15. J.K. Moore et al., Function of Dynein in Budding Yeast: Mitotic Spindle Positioning in a Polarized Cell, Cell Motil Cytoskeleton 66, 546 (2009).

16. N. Mizuno et al., Dynein and kinesin share an overlapping microtubule-binding site, $E M B O$ $J$ 23, 2459-2467 (2004). 
17. S. L. Reck-Peterson et al., Single-molecule analysis of dynein processivity and stepping behavior, Cell 126, 335-348 (2006).

18. K. Svoboda et al., Direct observation of kinesin stepping by optical trapping interferometry, Nature 365, 721-727 (1993).

19. R. D. Vale, Switches, latches, and amplifiers: common themes of G proteins and molecular motors, J Cell Biol 135, 291-302 (1996).

20. A. F. Neuwald et al., AAA+: A class of chaperone-like ATPases associated with the assembly, operation, and disassembly of protein complexes, Genome Res 9, 27-43 (1999).

21. L. Amos, A. Klug, Arrangement of subunits in flagellar microtubules, J Cell Sci 14, 523-549 (1974).

22. H. Li et al. . Downing, Microtubule Structure at $8 \AA$ A Resolution, Structure 10, 1317-1328 (2002).

23. W. Qiu et al., Dynein achieves processive motion using both stochastic and coordinated stepping, Nat Struct Mol Biol 19, 193-200 (2012).

24. M. A. DeWitt et al., Cytoplasmic Dynein Moves Through Uncoordinated Stepping of the AAA+ Ring Domains, Science 335, 221-225 (2012).

25. A. Yildiz et al., Kinesin walks hand-over-hand, Science 303, 676-678 (2004).

26. J. P. Erzberger, J. M. Berger, Evolutionary relationships and structural mechanisms of AAA+ proteins, Annual review of biophysics and biomolecular structure 35, 93-114 (2006). 
27. S. A. Burgess et al., Dynein structure and power stroke, Nature 421, 715-718 (2003).

28. A. J. Roberts et al., AAA+ Ring and linker swing mechanism in the dynein motor, Cell 136, 485-495 (2009).

29. I. R. Gibbons et al., Multiple nucleotide-binding sites in the sequence of dynein beta heavy chain, Nature 352, 640-643 (1991).

30. S. L. Reck-Peterson, R. D. Vale, Molecular dissection of the roles of nucleotide binding and hydrolysis in dynein's AAA domains in Saccharomyces cerevisiae, Proc Natl Acad Sci USA 101, 1491-1495 (2004).

31. Y. Takahashi et al., Multiple ATP-hydrolyzing sites that potentially function in cytoplasmic dynein, Proc Natl Acad Sci USA 101, 12865-12869 (2004).

32. T. Kon et al., The $2.8 \AA$ crystal structure of the dynein motor domain, Nature 484, 345-350 (2012).

33. C. Cho et al., Regulatory ATPase Sites of Cytoplasmic Dynein Affect Processivity and Force Generation, Journal of Biological Chemistry 283, 25839 (2008).

34. A. P. Carter et al., Crystal structure of the dynein motor domain, Science 331, 1159-1165 (2011).

35. T. Kon et al., X-ray structure of a functional full-length dynein motor domain, Nat Struct Mol Biol 18, 638 (2011). 
36. K. Imamula et al., The coordination of cyclic microtubule association/dissociation and tail swing of cytoplasmic dynein, Proc Natl Acad Sci USA 104, 16134-16139 (2007).

37. T. Kon et al., ATP hydrolysis cycle-dependent tail motions in cytoplasmic dynein, Nat Struct Mol Biol 12, 513-519 (2005).

38. H. Schmidt et al., Insights into dynein motor domain function from a 3.3-Å crystal structure, Nat Struct Mol Biol, - (2012).

39. M. A. Gee et al., An extended microtubule-binding structure within the dynein motor domain, Nature 390, 636 (1997).

40. M. P. Koonce, Identification of a Microtubule-binding Domain in a Cytoplasmic Dynein Heavy Chain, Journal of Biological Chemistry 272, 19714-19718 (1997).

41. C. V. Sindelar, K. H. Downing, The beginning of kinesin's force-generating cycle visualized at 9-A resolution, $J$ Cell Biol 177, 377-385 (2007).

42. I. R. Gibbons et al., The affinity of the dynein microtubule-binding domain is modulated by the conformation of its coiled-coil stalk, J Biol Chem 280, 23960-23965 (2005).

43. I. R. Gibbons, A. J. Rowe, Dynein: A Protein with Adenosine Triphosphatase Activity from Cilia, Science 149, 424-426 (1965).

44. T. Kon et al., Helix sliding in the stalk coiled coil of dynein couples ATPase and microtubule binding, Nat Struct Mol Biol 16, 325-333 (2009). 
45. R. Diaz et al., Fourier-Bessel reconstruction of helical assemblies, Meth Enzymol 482, 131165 (2010).

46. D. J. De Rosier, A. Klug, Reconstruction of three dimensional structures from electron micrographs, Nature 217, 130-134 (1968).

47. R. H. Wade et al., Toward understanding the structure and interactions of microtubules and motor proteins, Proteins 23, 502-509 (1995).

48. M. Kikkawa et al., $15 \AA$ Resolution Model of the Monomeric Kinesin Motor, KIF1A, Cell 100, 241-252 (2000).

49. E. M. E. Mandelkow et al., On the surface lattice of microtubules: helix starts, protofilament number, seam, and handedness, J Cell Biol 102, 1067-1073 (1986).

50. M. Kikkawa et al., Direct visualization of the microtubule lattice seam both in vitro and in vivo, J Cell Biol 127, 1965-1971 (1994)

51. E. Nogales et al., Structure of tubulin at $6.5 \AA$ and location of the taxol-binding site, Nature 375, 424-427 (1995).

52. H. Sui, K. H. Downing, Structural Basis of Interprotofilament Interaction and Lateral Deformation of Microtubules, Structure 18, 1022-1031 (2010).

53. G. M. Alushin et al., The Ndc80 kinetochore complex forms oligomeric arrays along microtubules, Nature 467, 805-810 (2010). 
54. C. V. Sindelar, K. H. Downing, An atomic-level mechanism for activation of the kinesin molecular motors, Proc Natl Acad Sci USA 107, 4111 (2010).

55. A. P. Carter et al., Structure and functional role of dynein's microtubule-binding domain, Science 322, 1691-1695 (2008).

56. A. J. Roberts et al., ATP-Driven Remodeling of the Linker Domain in the Dynein Motor, Structure 20, 1670-1680 (2012).

57. N. Mizuno et al., Three-dimensional structure of cytoplasmic dynein bound to microtubules, Proc Natl Acad Sci USA 104, 20832-20837 (2007).

58. A. Gennerich et al., Force-Induced Bidirectional Stepping of Cytoplasmic Dynein, Cell 131, 952-965 (2007).

59. S. Toba et al., Overlapping hand-over-hand mechanism of single molecular motility of cytoplasmic dynein, Proc Natl Acad Sci USA 103, 5741-5745 (2006).

60. L. Trabuco et al., Flexible fitting of atomic structures into electron microscopy maps using molecular dynamics, Structure 16, 673-683 (2008).

61. N. Grigorieff, FREALIGN: high-resolution refinement of single-particle structures, $J$ Struct Biol 157, 117-125 (2007).

62. M.P. Koonce and I. Tikhonenko, Functional elements within the dynein microtubule-binding domain, Mol Biol Cell 11, 523-529 (2000). 


\section{Chapter 2}

Structural basis for microtubule binding and release by dynein

William Redwine, Rogelio Hernandez-Lopez, Sirui Zou, Julie Huang, Samara ReckPeterson and Andres Leschziner 


\section{Contributions}

William Redwine initiated the project and performed biochemistry, sample preparation, and electron microscopy data collection. 3D reconstruction was performed by Rogelio HernandezLopez and William Redwine. Molecular dynamics was performed by Rogelio Hernandez-Lopez. Single molecule motility experiments were performed by Sirui Zou and Julie Huang. Andres Leschziner and Samara Reck-Peterson provided mentorship. 


\begin{abstract}
Cytoplasmic dynein is a microtubule-based motor required for intracellular transport and cell division. Its movement involves coupling cycles of track binding and release with cycles of force-generating nucleotide hydrolysis. How this is accomplished given the $\sim 25 \mathrm{~nm}$ separating dynein's track- and nucleotide-binding sites is not understood. Here, we present a subnanometer-resolution structure of dynein's microtubule-binding domain bound to microtubules by cryo-electron microscopy that was used to generate a pseudo-atomic model of the complex with molecular dynamics. We identified large rearrangements triggered by track binding and specific interactions, confirmed by mutagenesis and single molecule motility assays, which tune dynein's affinity for microtubules. Our results provide a molecular model for how dynein's binding to microtubules is communicated to the rest of the motor.
\end{abstract}




\section{Introduction}

Dyneins are ATP-driven molecular motors that move towards the minus ends of microtubules (MTs) (1). The superfamily includes axonemal dyneins, which power the movements of cilia, and those that transport cargo, which include cytoplasmic dyneins 1 (“cytoplasmic") and 2 (“intraflagellar") (2). The transport of organelles, ribonucleoprotein complexes and proteins by cytoplasmic dynein is required for cellular homeostasis, cell-cell communication, cell division, and cell migration (3) and defects in these processes result in neurological disease in humans (4). Despite cytoplasmic dynein's role in such diverse activities and recent advances in characterizing its structure and motility, many aspects of its molecular mechanism remain poorly understood.

The core of the cytoplasmic dynein holoenzyme is a homodimer of $\sim 500 \mathrm{kDa}$ motorcontaining subunits (Figure 2.1 A). The major functional elements include: (a) a "tail" domain required for dimerization and cargo binding (5); (b) the force-generating "head" or "motor domain" $(6,7)$, a ring containing six AAA + ATPase domains (8-10); (c) a "linker" connecting the head and tail, required for motility $(6,7,11,12)$; (d) the "stalk", a long antiparallel coiledcoil that emerges from AAA4 $(13,14)$, and (e) the MT-binding domain (MTBD), a small alphahelical domain at the end of the stalk responsible for binding the MT track (15-17). Unlike the other cytoskeletal molecular motors, kinesin and myosin, dynein does not have its ATPase and polymer track binding sites located within a single domain. With $25 \mathrm{~nm}$ separating AAA1, the main site of ATP hydrolysis, and the MTBD, an unresolved question is how dynein coordinates the cycles of nucleotide hydrolysis and MT binding and release required for its motion. 

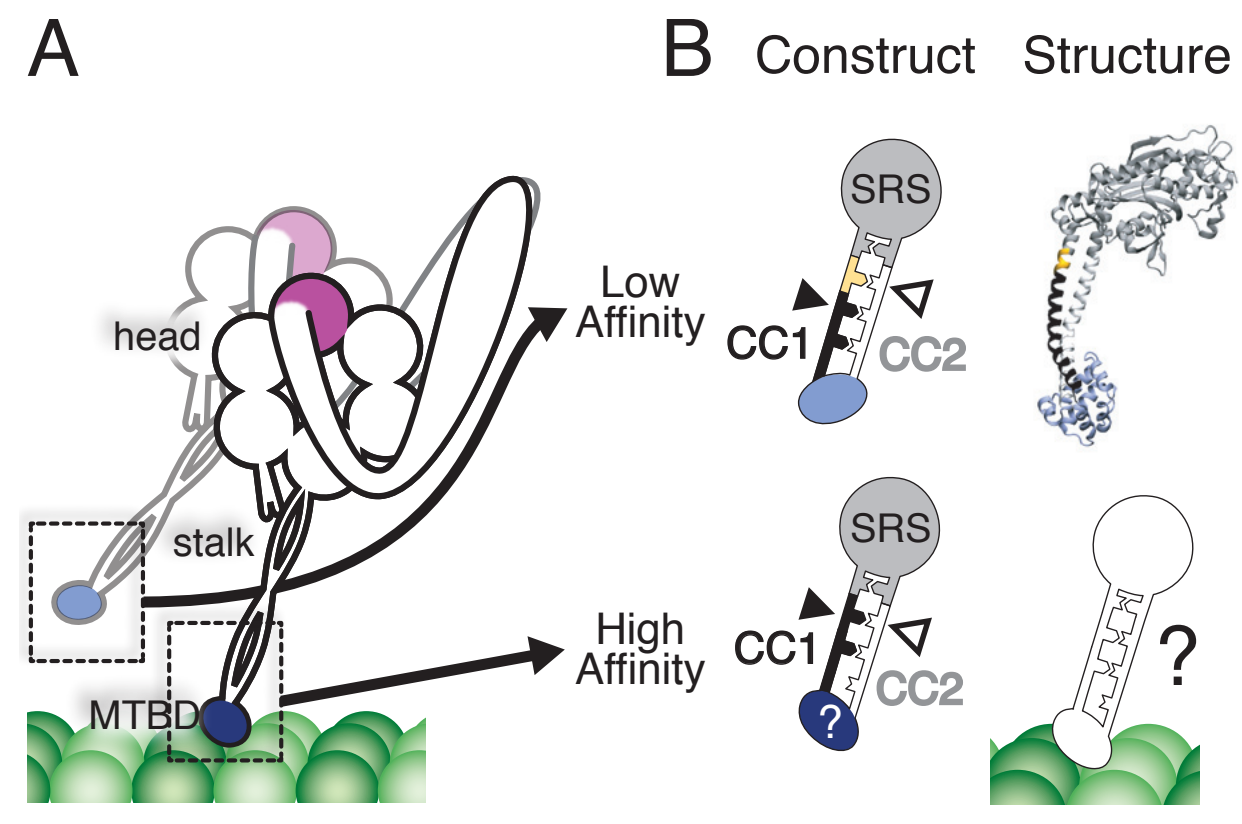

Figure 2.1. Towards a structural understanding of the helix sliding hypothesis (A) Schematic of dimeric cytoplasmic dynein. Major features relevant to this study are indicated. The MTBD is depicted in its low- (light blue) and high- (dark blue) affinity states during a step along the MT. (B) Schematic of the fusion constructs between the MTBD and seryl-tRNA synthetase (SRS) that fix the heptad registry of the stalk. The low-affinity construct has an additional 4 amino acids (yellow) inserted in $\mathrm{CC} 1$ (black) relative to the high-affinity construct. 
The mechanism coupling nucleotide hydrolysis to MT affinity has been suggested to be sliding in which the two helices in the stalk's coiled-coil adopt different registries by using alternative sets of hydrophobic heptad repeats (17-19). Dynein's stalk can be fixed in a specific registry by fusing it to a coiled-coil of known structure, such as that of seryl-tRNA synthetase (SRS) (18). Changing the length of the first stalk helix (CC1) relative to the second (CC2) shifts their alignment between high-affinity (" $\alpha$ ") and low-affinity (“ $\beta+$ ") registries, and alters the affinity of the MTBD for MTs by up to twenty-fold (Figure 2.1 B) (18). Engineered disulfide cross-linking in the monomeric dynein motor domain showed that its stalk explores multiple registries in solution, and that a given registry is coupled to a specific MT affinity. Fixing the stalk registry also uncoupled the nucleotide state of the head from MT affinity (19).

Understanding the molecular mechanism by which stalk sliding is coupled to nucleotide hydrolysis and MT affinity would be aided by a structure of dynein's MTBD bound to MTs. Crystal structures are available for a low-affinity dynein MTBD fused to SRS through a short segment of the stalk (Figure 2.1 B) (17), and an ADP-bound, presumably high-affinity dynein monomer (14). Only low-resolution structures of the MT-bound form have been reported (17, 20). Here we describe a cryo-electron microscopy (cryo-EM) reconstruction of the MTBD bound to MTs at sub-nanometer resolution, using a high-affinity version of the SRS-MTBD construct (18). 


\section{Materials and Methods}

\section{SRS-MTBD purification}

SRS-MTBD chimeric constructs were expressed in the Escherichia coli strain BL21 (DE3) containing the plasmid pLysS. Cells were grown in $8 \mathrm{~L}$ of LB broth at $37^{\circ} \mathrm{C}$ to an optical density at $600 \mathrm{~nm}$ of 0.5 . The cells were chilled on ice with periodic mixing until the temperature was 16 ${ }^{\circ} \mathrm{C}$. Protein expression was induced with $0.5 \mathrm{mM} \beta$-D-thiogalactopyranoside and the cells were further incubated at $16^{\circ} \mathrm{C}$ for $6 \mathrm{~h}$. All subsequent steps were performed at $4^{\circ} \mathrm{C}$ or on ice. Cells were harvested by centrifugation and were resuspended in $70 \mathrm{~mL}$ lysis buffer $(50 \mathrm{mM}$ Tris- $\mathrm{HCl}$, pH 8.0; 150 mM KOAc, 1 mM EDTA, 10\% glycerol, 20 mM imidazole, 5 mM $\beta$ mercaptoethanol, $1 \mathrm{mM}$ PMSF, and $1 \mu \mathrm{g} / \mathrm{mL}$ each pepstatin $\mathrm{A}$, aprotinin, benzamidine, and leupeptin). The cells were lysed by sonication ( 6 continuous pulses at $80 \%$ power, with 2 minutes between pulses) and the resulting lysate was centrifuged at $120,000 \mathrm{x} \mathrm{g}$ in a Ti45 rotor (Beckman) for $30 \mathrm{~min}$ at $4^{\circ} \mathrm{C}$. The cleared lysate was applied to a $10 \mathrm{~mL}$ Ni-NTA agarose column (Qiagen) by gravity. The column was then washed with $300 \mathrm{~mL}$ lysis buffer. Bound protein was then eluted with a linear imidazole gradient from $50 \mathrm{mM}$ to $500 \mathrm{mM}$. Peak fractions containing the SRS-MTBD fusion, as determined by SDS-PAGE analysis, were pooled, dialyzed for $4 \mathrm{~h}$ against lysis buffer, and concentrated to $\sim 15 \mathrm{mg} / \mathrm{mL}$ with Ultracell concentration columns with a molecular weight cut-off of $30 \mathrm{kDa}$ (Millipore). A fraction of this protein was then further purified on a Superdex 200 16/60 gel filtration column equilibrated in lysis buffer. A monodisperse peak was isolated and concentrated to $\sim 4 \mathrm{mg} / \mathrm{mL}$ as described above. 


\section{Tubulin polymerization}

Highly purified, glycerol-free tubulin (Cytoskeleton, Inc.) was resuspended in BRB80 (80 mM PIPES-KOH, pH 6.8; $1 \mathrm{mM} \mathrm{MgCl2,} 1$ mM EGTA, 1 mM DTT) to a concentration of 10 $\mathrm{mg} / \mathrm{mL}$. The resuspended tubulin was centrifuged at $100,000 \mathrm{xg}$ in a TLA 100 rotor (Beckman) for $30 \mathrm{~min}$ at $4{ }^{\circ} \mathrm{C}$ to remove aggregates, and the protein concentration was determined using an extinction coefficient of $1.15(\mathrm{mg} / \mathrm{mL})^{-1}$. Tubulin was then polymerized with a stepwise addition of taxol as follows: $20 \mu \mathrm{L}$ of tubulin stock was thawed quickly and placed on ice. To this $10 \mu \mathrm{L}$ of BRB80 supplemented with $3 \mathrm{mM} \mathrm{GTP}$ was added and the mixture was transferred to a $37^{\circ} \mathrm{C}$ water bath. After 15, 30, and 45 minutes, additions of $0.5,0.5$, and $1.0 \mu \mathrm{L}$ of $2 \mathrm{mM}$ taxol were added by gentle swirling. The mixture was then incubated for an additional $1 \mathrm{~h}$ at $37^{\circ} \mathrm{C}$.

\section{Grid preparation and imaging}

Purified SRS-MTBD protein was dialyzed for $4 \mathrm{~h}$ against lysis buffer without glycerol and added salts (cryo buffer: $50 \mathrm{mM}$ Tris- $\mathrm{HCl}, \mathrm{pH}$ 8.0, $1 \mathrm{mM} \mathrm{MgCl2,} 1 \mathrm{mM}$ EGTA, $1 \mathrm{mM}$ DTT) in a spin dialyzer (Harvard Apparatus) fitted with a $10 \mathrm{kDa}$ MWCO membrane. The protein was recovered and centrifuged at $20,000 \mathrm{x}$ g in a TLA 100 rotor (Beckman) for $30 \mathrm{~min}$ at $4{ }^{\circ} \mathrm{C}$ to remove aggregates. C-flat 2/2-2C holey carbon grids (Protochips) were glow-discharged for $20 \mathrm{~s}$ at $30 \mathrm{~mA}$ in an Edwards carbon evaporator. MTs were diluted to $0.5 \mathrm{mg} / \mathrm{mL}$ in cryo buffer supplemented with $100 \mu \mathrm{M}$ taxol, and $4 \mu \mathrm{L}$ were added to a grid and allowed to absorb for 30 sec. The solution was manually blotted from the side with a torn Whatman \#1 filter paper. Next, $4 \mu \mathrm{L}$ of dialyzed SRS-MTBD was added and allowed to bind to the MTs for 1 min. The solution was blotted manually again, and the process of addition and blotting of SRS-MTBD was 
repeated for a total of three times. The final blotting was done inside the humidity chamber of a Vitrobot Mark IV (FEI) set at $22^{\circ} \mathrm{C}$ and $100 \%$ humidity. The grids were then rapidly plunged into a liquid nitrogen-cooled ethane slush. Grids were stored in liquid nitrogen until imaging.

Grids were transferred to a Gatan 626 cryo-holder, and micrographs were collected on film (SO-163, Kodak) in a Tecnai F20 electron microscope (FEI) operating at $120 \mathrm{keV}$, and nominal magnification of 62,000. Final accumulated electron doses ranged between 15 and 20 electrons/ $\AA^{2}$. The actual magnification of 63,377 was calculated using tobacco mosaic virus layer lines as a standard. Film was developed using full strength D9 developer (Kodak) for 12 min. Each film was analyzed visually on a light box for drift; those without noticeable drift were digitized on an Aztek Plateau flatbed scanner (Aztek, Inc.) with a 6.35 micron step size, resulting in a final pixel size of $0.994 \AA$. Images were then selected for processing on the basis of high decoration, straight MTs, and the absence of crystalline ice and drift.

\section{Image processing and three-dimensional reconstruction}

Square segments of 720 pixels were extracted from micrographs containing straight MTs using BOXER (21) and the "helix" option with 240 pixel overlap. Pixel intensities were normalized and segments were decimated six-fold to a pixel size of $5.964 \AA$. Reference-free twodimensional classification was performed as described $(22,23)$. This method has been shown to sort MT segments on the basis of degree of decoration and protofilament number (PF). After analyzing the power spectrum and one-dimensional projection of class averages, good quality boxes were assigned a PF number of 13,14 or 15 . We assigned PF number based on the consensus of PF number assignment for all boxes extracted from each individual MT. Out of 577 
MTs, 189 13-PF and 375 14-PF MTs were selected for further processing.

The primary PF number population in our in vitro assembled MT preparations was 14 . To maximize the data used for reconstruction, custom SPIDER scripts $(24,25)$, previously used in subnanometer reconstructions of 13 PF MT complexes $(24,26)$, were adapted to 14 PF MTs. Initially, $13 \mathrm{PF}$ and $14 \mathrm{PF}$ MTs were independently processed to validate our implementation. We obtained essentially the same structure at a resolution of $20 \AA$, at which point the $13 \mathrm{PF}$ reconstruction stopped improving (data not shown).

In our implementation, a PDB model of the SRS-MTBD-MT (PDB 3ERR (17) and 1JFF (27)) complex was used to generate an initial low resolution volume from which a set of initial projection references was generated to assign the orientation of individual $80 \AA$ experimental segments. The astigmatism and defocus values used to correct the contrast transfer function of the microscope were obtained from micrographs using CTFFIND3 (28) and were used during the reconstruction step. Three cycles of alignment using the SPIDER custom scripts with 3 rounds of reconstruction with FREALIGN (26) in each cycle, yielded a final $12.0 \AA$ resolution structure (FSC at 0.143 , a criterion shown to be reliable $(29,30)$ for structures refined with FREALIGN. Reconstructions in cycles 1 and 2 were done with data decimated three-fold. In the last cycle the reconstruction was carried out at finer pixel sampling (1.988 $\AA$ ). Within each cycle the reconstruction with the highest resolution was used to create new references that were used for a subsequent cycle of alignment. Helical parameters for three-dimensional reconstruction were obtained using the hsearch_lorentz program (31). The helical rise and angle converged to $9.26 \AA$ and $-25.76^{\circ}$ respectively. For three-dimensional reconstruction, a recent implementation of a helical symmetry operator in FREALIGN was used (23). In this algorithm, each $80 \AA$ segment is 
included multiple times in the reconstruction, using the Euler angles and shifts, to generate symmetrically equivalent views. Due to the presence of the seam in the 14 PF MTs, each segment was inserted 14 times. As previously described $(24,25)$, the symmetrization step causes the output reconstruction from FREALIGN to contain a single PF with all asymmetric subunits in registry. A SPIDER script was used to rebuild the MT containing the correct seam position, as described (32). Our final reconstruction contains data from 191 MTs equivalent to $10,41980 \AA$ segments or 145,866 individual tubulin dimers. Visual inspection of our reconstruction after sharpening the map revealed that the resolution for the portion of the map corresponding to the SRS is lower than the portion containing the MT-MTBD interface. We applied a cylindrical soft mask to exclude the density corresponding to the SRS and measured the resolution of the map in its absence. The resolution of the MT-MTBD map calculated from half volumes of the final reconstruction is $9.7 \AA$ (FSC 0.143 criterion), which is more consistent with our ability to see secondary structural elements in portions of the map. The final map was sharpened using Gaussian low-pass and high-pass filters at 9.75 and $30 \AA$, respectively, and the high-resolution amplitudes were scaled using a b-factor of $-200 \AA^{2}$ using the program BFACTOR with the cosine edge mask option with a radius of $9.5 \AA$.

\section{Flexible fitting and targeted molecular dynamics}

Our Experimental 3D map was segmented to obtain a density comprising an asymmetric tubulin dimer unit using UCSF Chimera (33). The atomic resolution structures for the MTBD (PDB 3ERR (17)), excluding 4 residues (3360 to 3363); and tubulin, obtained after flexible fitting into a MT density (34), were used for rigid body docking using UCSF Chimera. 
Molecular dynamics flexible fitting (MDFF) $(35,36)$ and targeted molecular dynamics (TMD)

(37) were utilized to obtain a pseudo-atomic model of the high affinity MTBD-MT complex.

Initially, the MTBD-Tubulin system was minimized for 2000 steps and then a vacuum MDFF simulation was run for $100 \mathrm{~ns}$ at $300 \mathrm{~K}$, using a dielectric constant of 80 and a force scaling factor $(\xi)$ of $0.5 \mathrm{kcal} / \mathrm{mol}$. Restraints on secondary structure elements and chiral atoms were used as described (36). After the simulation in vacuum, the system was embedded in a box of water molecules using the VMD plugin "Solvate" with a $10 \AA$ distance from the closest protein atoms to the box edge. The VMD plugin "Autoionize" was used to randomly place potassium and chloride ions that simulate a final $\mathrm{KCl}$ concentration of $0.05 \mathrm{M}$. All-atom molecular dynamics simulations were performed using the software NAMD $1.7 \mathrm{~b} 2(38)$, the CHARMM27 force field with CMAP correction terms (39) and the TIP3P water model (40). The same parameters for the GDP and GTP molecules were used as described(34). The system was minimized for 2000 steps followed by $10 \mathrm{~ns}$ of MDFF at $300 \mathrm{~K}$ using a force-scaling factor $(\xi)$ of $0.5 \mathrm{kcal} / \mathrm{mol}$ and constraints on secondary structure elements and chiral atoms. During MDFF simulations, the temperature was kept constant at $300 \mathrm{~K}$ using Langevin dynamics with a damping coefficient of $5 \mathrm{ps}-1$. The long-range electrostatic interactions were calculated using the Particle Mesh Ewald method (PME) and the van der Waals interactions were computed with a 10 $\AA$ A cutoff using periodic boundary conditions. After the MDFF simulation in explicit solvent the system was equilibrated for $10 \mathrm{~ns}$ in the NPT ensemble (see below).

After these simulations, we observed a rearrangement of the helices that form the MTBD, with no apparent shift in the registry of the stalk. As our cryo-EM data was collected with a construct known to exist in the high affinity registry, TMD (37) was performed on the C $\alpha$ atoms 
of residues 3264-3280 using a $200 \mathrm{kcal} / \mathrm{mol} \AA 2$ force constant. In TMD, the positions of selected atoms are driven into the target positions by using an additional potential energy term in which the root-mean-squared deviation between the simulated and target position is minimized during the simulation. Using this approach, the registry shift from low- to high-affinity was obtained, whereas the rest of the atoms were still driven by the cryo-EM map using MDFF. Fifteen ns of MDFF/TMD at $300 \mathrm{~K}$ using a force-scaling factor $(\xi)$ of $0.5 \mathrm{kcal} / \mathrm{mol}$ and constraints on secondary structure elements and chiral atoms were carried out.

To confirm that the structure for the dynein high affinity MTBD state does not depend on the multistep simulations that we carried out, we ran additional simulations as follows: the starting crystal structures of tubulin and the low-affinity MTBD (described above) were rigid body docked into our density, the output system was solvated and ions were randomly placed as described above. The system was minimized for 1000 steps followed by $20 \mathrm{~ns}$ of MDFF/TMD at $300 \mathrm{~K}$ using a force-scaling factor $(\xi)$ of $0.5 \mathrm{kcal} / \mathrm{mol}$ and constraints on secondary structure elements and chiral atoms. A similar high affinity MTBD structure was obtained.

\section{Equilibration simulations}

After the MDFF/TMD simulations, the system was equilibrated for $20 \mathrm{~ns}$ in the NPT ensemble at $300 \mathrm{~K}$ and $1 \mathrm{~atm}$ using the Nose-Hoover Langevin piston with a $200 \mathrm{fs}$ decay period and a $50 \mathrm{ps}^{-1}$ damping time constant for the pressure and $0.1 \mathrm{ps}^{-1}$ for the temperature. PME was used for long-range electrostatic interactions and a $12 \AA$ cutoff (switching function at $10 \AA$ ) for van der Waals interactions. A uniform integration step of $2 \mathrm{fs}$ was used during these simulations. The backbone coordinates of tubulin and residues 3411 to 3427 in the dynein MTBD were 
constrained using harmonic forces with a force constant of $1 \mathrm{kcal} / \mathrm{mol} \AA^{2}$ to keep fixed the center of mass of the molecules. During the 10 first ns of these simulations, TMD with 100 and $50 \mathrm{kcal} /$ mol $\AA^{2}$ force constants, on the same atoms described above, were used. All TMD constraints were released during the last $10 \mathrm{~ns}$.

Additional simulations were run using the low-affinity MTBD and tubulin structures without any external force, and using TMD to induce the registry shift, both without the presence of our experimental map. The systems were prepared using the tubulin coordinates obtained after equilibration of the high-affinity MTBD-Tubulin complex (see above) and the crystal structure coordinates of the low-affinity construct (PDB 3ERR (17)). The positions of residues 3388 to 3426 were used to align the dynein MTBD low- and high-affinity structures, as they were unchanged in both conformations. The MTBD-tubulin complex coordinates were embedded in a box of explicit water molecules and $\mathrm{KCl}$ ions as described above. In the case of the TMD simulations, restraints on secondary structure elements and chiral atoms were used to avoid structural distortions of the protein. Fifteen ns of TMD simulations were carried out with the same parameters as the MDFF/TMD simulation described above. The simulation of the lowaffinity MTBD structure on MTs included 100 ps simulation with constraints in all backbone atoms with a force constant of $1 \mathrm{kcal} / \mathrm{mol} \AA^{2}$ followed by a $15 \mathrm{~ns}$ equilibration simulation in the NPT ensemble as described above. In the absence of the map forces, the repositioning of the helices $\mathrm{H} 1, \mathrm{H} 3$ and $\mathrm{H} 4$ was not observed during the simulated time scale, though favorable interactions between the dynein low-affinity conformation and tubulin were detected.

\section{Analysis of trajectories}


The last $10 \mathrm{~ns}$ of equilibration simulations were used to perform trajectory analysis using VMD (41). The RMSD of the high- and low-affinity MTBD equilibrated structures was measured and its convergence supports the stability of the conformers. Salt bridges were monitored if the donor-acceptor distance was less than $3.2 \AA$. Hydrogen bonds were counted if the donor-acceptor distance was less than $3.0 \AA$ with a $20^{\circ}$ cutoff for the angle formed by the donor, hydrogen, acceptor. Figures depicting molecular structures were created with UCSF Chimera (33) and VMD (41).

\section{Comparison of our experimental high-affinity map with synthetic maps}

To compare the conformational changes described by our MD-generated pseudo-atomic models with our experimental density, we generated $10 \AA$ resolution synthetic maps of the low and high affinity conformations using SPIDER and fitted them into our experimental cryo-EM map. At this resolution secondary structure elements such as alpha helices are visible as discrete regions of density in both our synthetic and experimental maps. We calculated the crosscorrelation coefficient between each of the synthetic maps and our experimental cryo-EM map (36). The higher similarity between the synthetic map of the high-affinity state and our experimental density (CCC 0.751$)$ when compared to the low-affinity conformation (CCC 0.648 ) indicates that our map describes the large conformational changes seen in our pseudo-atomic models. In particular, the density corresponding to $\mathrm{H} 1$ in the low-affinity synthetic map is completely absent in our experimental EM density, whereas the high-affinity synthetic map shows a very good match to the experimental density. 
Flexible fitting of Kon et al. MTBD

We performed MDFF using our EM map and the atomic coordinates of the MTBD of an ADP-bound dynein monomer (PDB 3VKH (14)). This structure has the stalk's coiled-coil in the $\alpha$ registry but its MTBD has the same conformation as the low-affinity MTBD previously reported by Carter et al. (17). The crystal structures of MDFF tubulin (described above) and the MTBD (residues 3352 to 3491) were rigid-body docked into our density, the initial coordinates for the complex were solvated, and ions were randomly placed as described above. The system was minimized for 2000 steps followed by $12 \mathrm{~ns}$ of MDFF at $300 \mathrm{~K}$ using a force-scaling factor $(\xi)$ of $0.5 \mathrm{kcal} / \mathrm{mol}$ and constraints on secondary structure elements and chiral atoms. A similar high-affinity conformation for the MTBD region (backbone RMSD $3.3 \AA$ relative to residues 3285 to 3402 in the high-affinity conformation described above in this study) was obtained. During this simulation we observed that the final position of $\mathrm{CC} 1$ deviates from the one observed in our previous simulations, likely because of the absence of coordinates for the $\mathrm{CC} 2$ residues in the distal portion of the coiled-coil of the crystal structure reported by Kon et al.

Two-dimensional analysis of monomeric S. cerevisiae dynein bound to microtubules

Monomeric dynein protein was purified as described for dimeric dynein (11), bound to MTs, and imaged under cryogenic conditions. MTs were polymerized and grids were prepared as described above. Images were collected on a US4000 4k x 4k CCD (Gatan) in a Tecnai F20 electron microscope (FEI) operating at $200 \mathrm{keV}$, and nominal magnification of 50,000. Square segments of $600 \times 600$ pixels with $80 \%$ overlap were extracted and alignment and classification of data binned by 3 (final pixel size of $6.42 \AA$ ) was performed using a previously reported 
strategy (22).

Estimating the conformation of monomeric dynein on microtubules

We noticed that rigid body docking of the Kon et al. dynein monomer crystal structure (14) via its MTBD into our reconstruction results in the dynein head clashing with the MT. Using our MDFF-generated Kon et al. MTBD (described above) we ran additional targeted molecular dynamics simulations to position $\mathrm{CC} 1$ according to our high-affinity model (to correct for the deviation in $\mathrm{CC} 1$ due to the absence of $\mathrm{CC} 2$ in the Kon et al. structure). We then modeled the structure of the entire monomer using the remaining portion of the monomer crystal structure (PDB 3VKH (14)).

\section{S. cerevisiae strain construction}

Mutations were inserted into a truncated dynein construct that is dimerized with GST as described previously (11). All mutations were verified by DNA sequencing. The wild type form of this construct has been shown to have near identical properties as full-length cytoplasmic dynein (11).

\section{Yeast dynein purification}

Cultures of S. cerevisiae for protein purification were grown, harvested, and frozen as previously described (11). Dynein constructs were purified via their ZZ tags and labeled with HaloTag-TMR (Promega) as described (11). 


\section{Total Internal Reflection Fluorescence Microscopy (TIRF)}

Fluorescently-labeled molecules were visualized using an Olympus IX-81 TIRF microscope with a 100X 1.45 N.A. oil immersion TIRF objective (Olympus) and two CW diodepumped solid state lasers $(491 \mathrm{~nm}$ and $561 \mathrm{~nm}$, Cobolt). Laser power at the objective was $1.5-2$ $\mathrm{mW}$. Images were recorded with a $100 \mathrm{~ms}$ exposure on a back-thinned electron multiplier CCD camera (Hamamatsu), controlled by Metamorph software.

Single-molecule motility assays of mutant dyneins (Figure 2.13, 14, 15, 16) were performed with MTs containing HiLyte Fluor ${ }^{\mathrm{TM}}$ 488- and biotin-labeled tubulin (Cytoskeleton, Inc.). MTs were prepared as described (42). Single-molecule motility assays that addressed the effect of increasing ionic strength (Figure 2.10) on dynein motility utilized Cy5-labeled axonemes prepared as described (11). All assays utilized a flow chamber as described $(11,43)$, with the following modification: TMR-labeled dynein motors were added to motility chambers in the presence of motility buffer [30 mM HEPES (pH 7.2), 2 mM MgOAc, 1 mM EGTA, 10\% glycerol, $1 \mathrm{mM}$ DTT, $1 \mathrm{mM} \mathrm{Mg-ATP,} \mathrm{and} \mathrm{an} \mathrm{oxygen} \mathrm{scavenger} \mathrm{system]} \mathrm{(11).} \mathrm{To} \mathrm{determine} \mathrm{the}$ effect of ionic strength on dynein motility, assay buffer was supplemented with $20,40,60$, or 80 mM KAc (Figure 2.10), or $50 \mathrm{mM} \mathrm{KAc} \mathrm{(Figure} 2.13,14,16$ ), or $50 \mathrm{mM} \mathrm{KAc}+50 \mathrm{mM} \mathrm{KCl}$ (Figure 2.16). Images were recorded every $2 \mathrm{~s}$ for $10 \mathrm{~min}$, and velocities and run lengths were calculated from kymographs generated in Image $(\mathrm{NIH})$ as described (11). Run lengths were not corrected for photobleaching of fluorophores, as this rate was determined to be negligible (data not shown).

Preparation of tubulin lacking C-terminal E-hooks 
Polymerized biotin-labeled MTs (described above) were incubated with subtilisin at the ratio of $5: 1$ (w/w of tubulin:subtilisin) for $1 \mathrm{~h}$ at $37^{\circ} \mathrm{C}$. The reaction was stopped by adding $2 \mathrm{mM}$ phenylmethylsulfonyl fluoride (PMSF). The digestion was monitored by SDS-PAGE to confirm complete cleavage. TIRF microscopy with subtilisin treated MTs was performed as described above. 
Table 2-1. Yeast strains used in this study. All yeast strains are based on the W303 background. Note that the following relationship between Saccharomyces cervisiae $(S c)$ and Mus musculus $(M m)$ dynein residues: $S c$ E3107 $=M m$ E3289 and $S c$ E3197 $=M m E 3378$.

\begin{tabular}{|c|c|c|c|}
\hline Strain ID & Genotype & Source & Figure \\
\hline RPY98 & $\begin{array}{l}\text { MATa, his3-11,5; ura3-52 leu2-3,112; ade2-1, trp-1, } \\
\text { pep4A::HIS5, PAC11-13Myc-TRP, pGal-ZZ-Tev- } \\
\text { GFP-HA-Dyn } 1_{331 k D a}\end{array}$ & (11) & Fig. 2-7 D, E \\
\hline RPY208 & 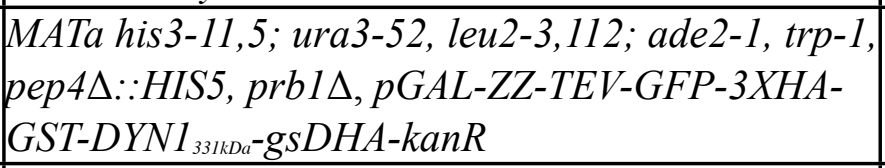 & (11) & $\begin{array}{l}\text { Fig. 2-13 A, } \\
\text { B, Fig. 2-14, } \\
\text { 2-15, 2-16 } \\
\end{array}$ \\
\hline RPY237 & 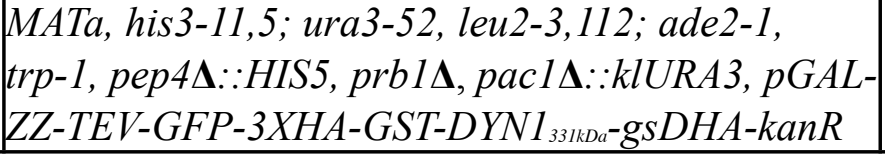 & (11) & Fig. 2-10 \\
\hline RPY1217 & 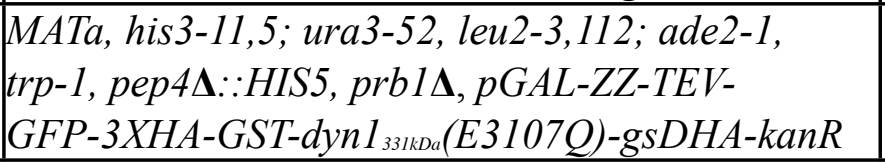 & this study & $\begin{array}{l}\text { Fig. 2-13 A, } \\
\text { B, Fig. 2-14 }\end{array}$ \\
\hline RPY1218 & 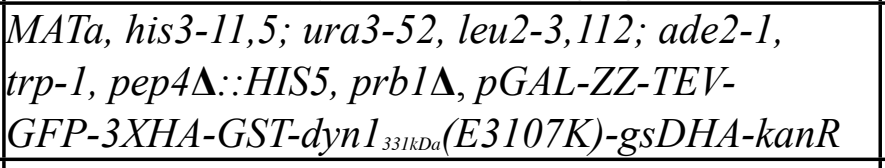 & this study & $\begin{array}{l}\text { Fig. 2-13 A, } \\
\text { B, Fig. 2-14, } \\
\text { 2-15, 2-16 } \\
\end{array}$ \\
\hline RPY1233 & 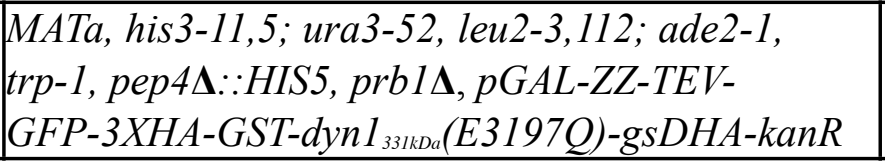 & this study & $\begin{array}{l}\text { Fig. 2-13 A, } \\
\text { B, Fig. 2-14 }\end{array}$ \\
\hline RPY1235 & 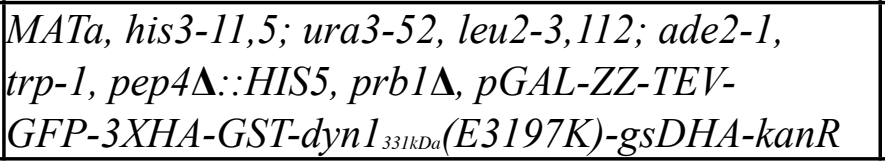 & this study & $\begin{array}{l}\text { Fig. 2-13 A, } \\
\text { B, Fig. 2-14, } \\
\text { 2-15, 2-16 }\end{array}$ \\
\hline RPY1247 & 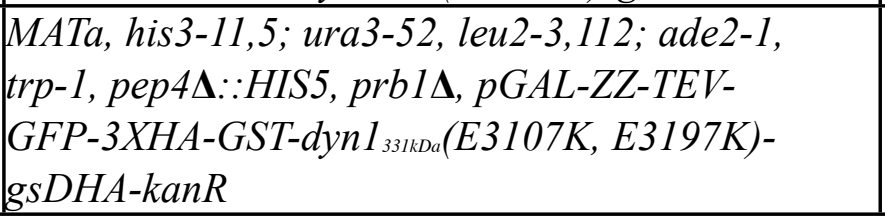 & this study & Fig. 2-15 \\
\hline
\end{tabular}




\section{Results}

We collected images of MTs highly decorated with the SRS-MTBD under cryogenic conditions (Figure 2.2 A, Figure 2.3 A), and adapted an image processing method $(24,32)$ to solve its structure bound to 14-protofilament MTs. The SRS-MTBD binds to $\alpha$-tubulin and $\beta$ tubulin at the intradimer interface and is positioned to the side of the protofilament, as previously reported $(17,20)$ (Figure 2.2 B, Figure 2.3 B). In the reconstruction, the portion including the MT, the MTBD and the beginning of the stalk has a resolution of $9.7 \AA$ (Figure $2.3 \mathrm{D}$ ), where $\alpha$ helices become visible.

We used molecular dynamics (MD) and our cryo-EM reconstruction to obtain pseudoatomic models of the low- and high-affinity states of the MTBD bound to MTs. First, we rigidbody docked the atomic-resolution structures of the tubulin dimer $(27,34)$ and the low-affinity MTBD into our map (Figures 2.4 A, C, and 2.5 A) and used MD to resolve steric clashes between a helix (H1) in the MTBD and the MT (Figures 2.4 A, C and 2.5 A, B). We then performed explicit-solvent molecular dynamics flexible fitting (MDFF) to shift the low-affinity MTBD structure to the high-affinity conformation in our reconstruction (Figure 2.5 C). In addition to the MD force field, MDFF uses a potential energy term derived from the experimental map and restraints on secondary structure to drive conformational changes that better fit the map $(35,36)$. The initial MDFF model agreed well within the experimental density of the MTBD (Figure 2.5 C); however, the stalk remained in the low-affinity $\beta+$ registry present in the starting model. MDFF likely did not shift the registry of the coiled-coil due to the lower resolution at the tip of the stalk segment (Figure 2.3); a movement of the CC1 helix to the $\alpha$ 


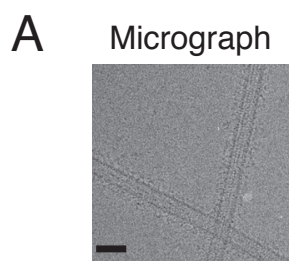

B Class Average
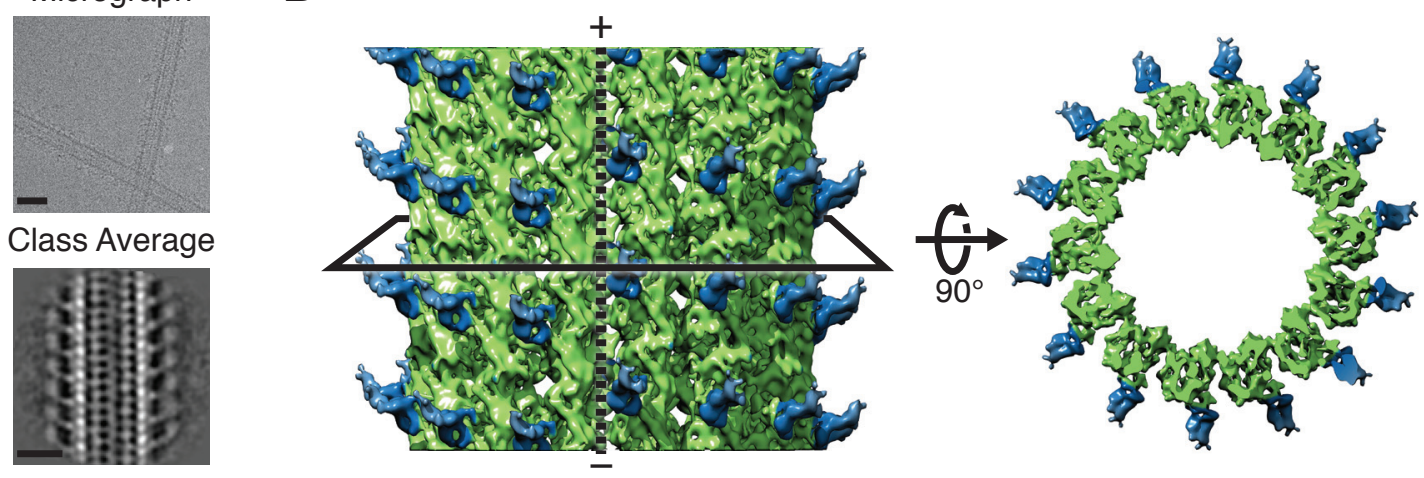

Figure 2.2. Sub-nanometer MTBD-MT reconstruction. (A) Cryo-EM image of MTs highly decorated with the high-affinity SRS-MTBD construct (top) and a class average generated from segments of decorated 14-protofilament MTs (bottom). Scale bars: $25 \mathrm{~nm}$. (B) Threedimensional reconstruction of the MTBD-MT complex, filtered to the calculated resolution of $9.7 \AA$ (Figure 2.3). The black solid line represents a slice through the volume, which is shown on the right viewed from the minus end of the MT. The MT polarity is indicated and the dashed line shows the location of the MT seam. The SRS has been omitted due to its lower resolution 

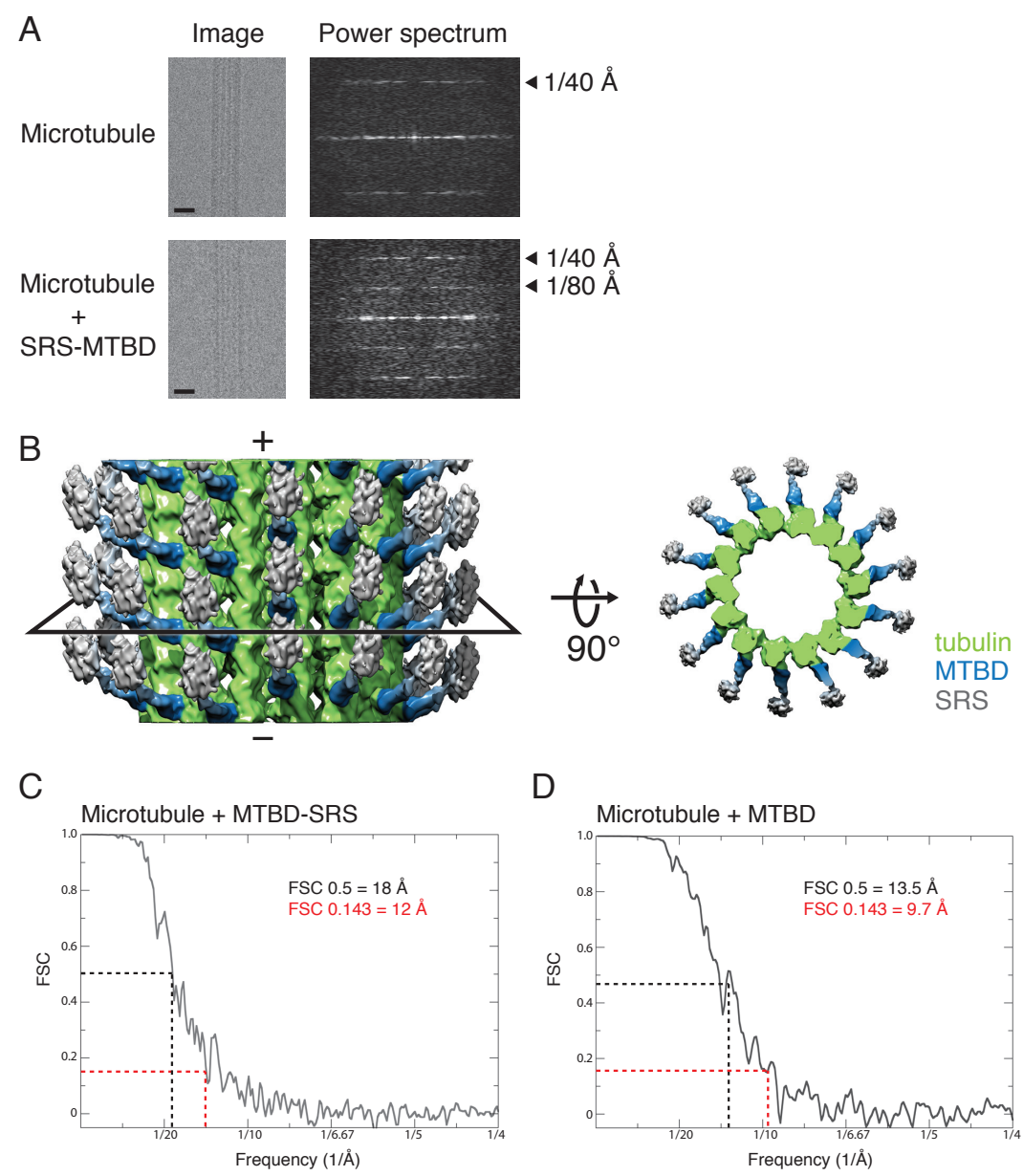

Figure 2.3. Complete reconstruction of the high-affinity SRS-MTBD construct bound to a MT. (A) Left: Images containing MTs only (top) and MTs highly decorated with the high-affinity SRS-MTBD (bottom). Scale bar represents $20 \mathrm{~nm}$. Right: Corresponding power spectra of the MTs. Note the layer lines at $1 / 40 \AA$ and $1 / 80 \AA$, which correspond to the repeated units of tubulin monomers and SRS-MTBD, respectively. (B) Left: A segment of the MT + SRS-MTBD reconstruction low-pass filtered to $12 \AA$. MT polarity is indicated by plus and minus signs. Right: A slice taken through the volume, related to the complete reconstruction by a $90^{\circ}$ rotation, such that the viewer is looking down the MT from the minus end. The color of each component is indicated and refers to both views. (C) The estimated resolution of the complete volume, including the SRS, is $12 \AA$, using the 0.143 criterion (red dashed line). (D) The estimated resolution of the volume containing only the MT and MTBD is $9.7 \AA$, using the 0.143 criterion (red dashed line). The discrepancy in resolutions is likely due to a relatively less constrained SRS domain decreasing the overall resolution of the structure. 


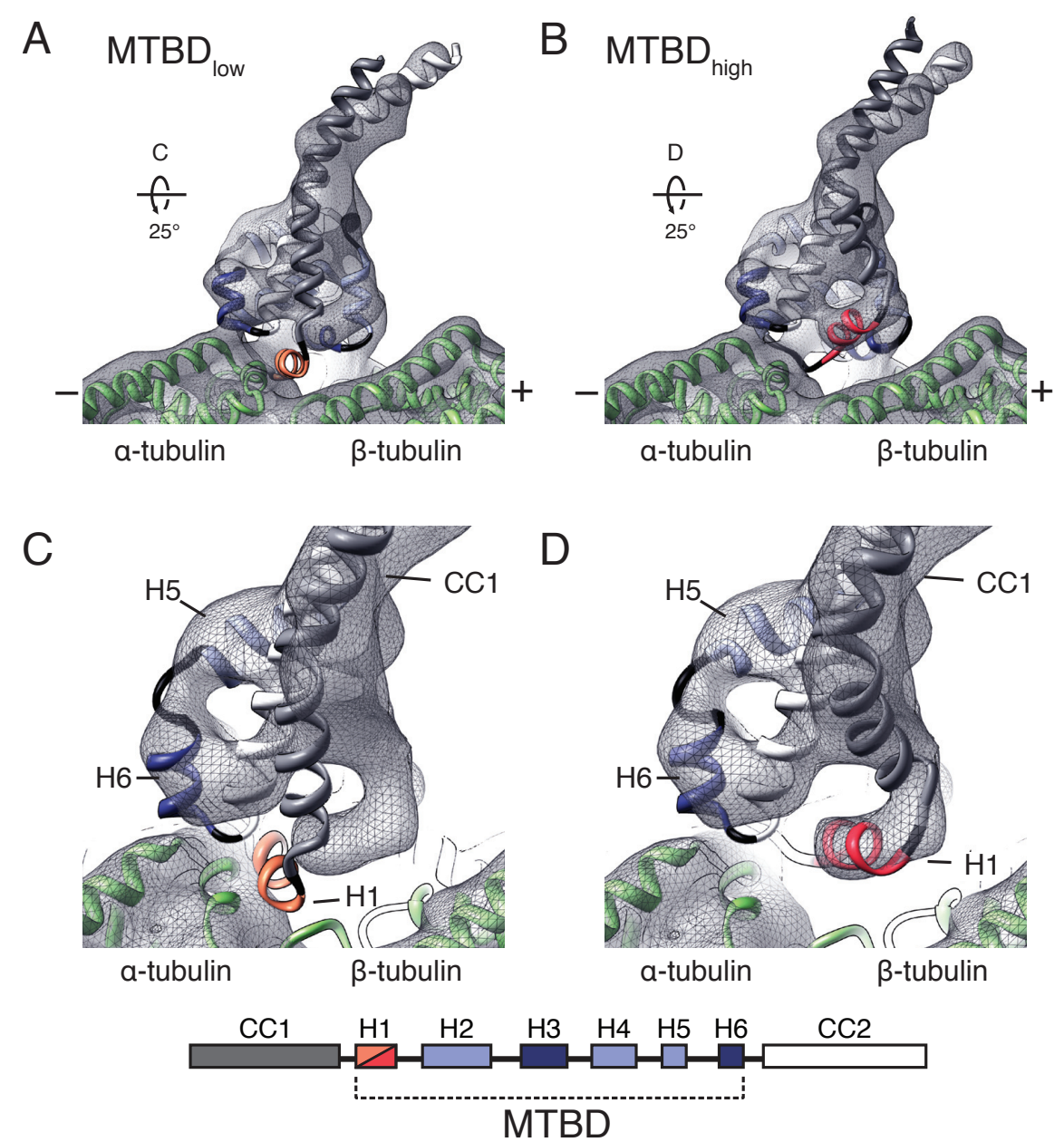

Figure 2.4. The high-affinity, MT-bound state of the dynein MTBD is characterized by repositioning of helices $\mathrm{H} 1$ and $\mathrm{CC} 1$. (A) Rigid-body docking of the low-affinity MTBD structure into our cryo-EM density. (B) Pseudo-atomic model of the high-affinity MTBD bound to MTs generated by Molecular Dynamics Flexible Fitting (MDFF) and Targeted Molecular Dynamics (TMD) (see text for details). (C) Close-up of the structure shown in (A), with its orientation indicated in panel (A). (D) Close-up of the structure shown in (B), with its orientation indicated in panel (B). The cryo-EM map is shown as a transparent grey mesh. The MTBD is colored following the scheme shown at the bottom of the figure and structural elements are indicated in the different views. H1 (orange/red) is the element with the largest movement in the transition to the high-affinity conformation; H3 and H6 (dark blue) are major contact points with the MT (green). $\alpha$ - and $\beta$-tubulin are indicated (green). MT polarity is indicated in panels A and B. H1 protrudes from the cryo-EM map and clashes with the MT in the rigid-body docked low-affinity state (A and C). 


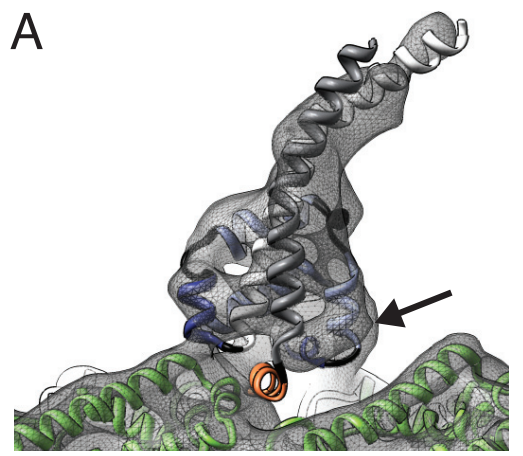

Low affinity rigid body docking CCC 0.648

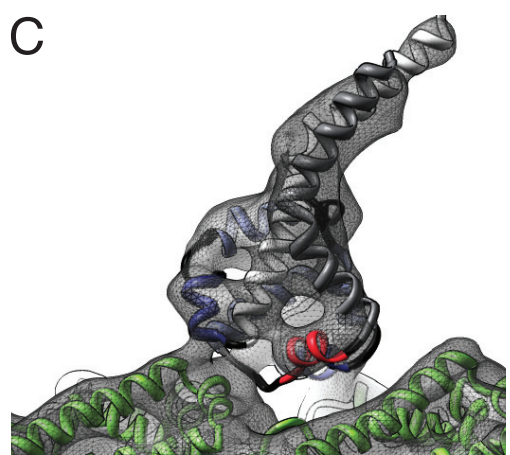

High affinity MDFF CCC 0.737

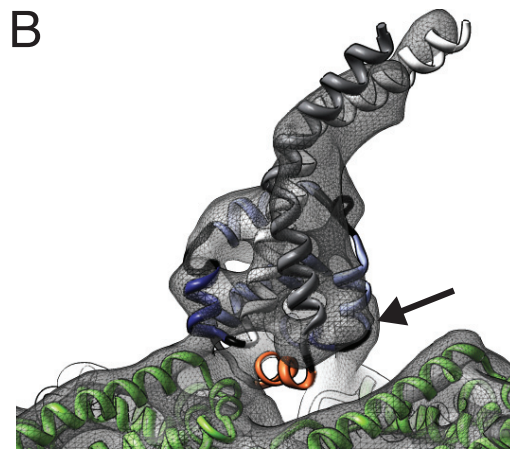

Low affinity equilibrated CCC 0.716

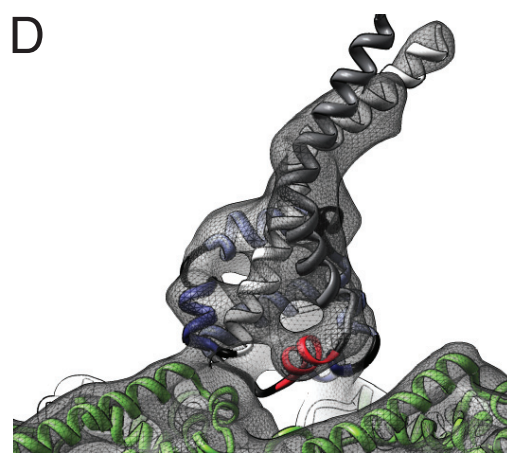

High affinity MDFF + TMD CCC 0.751

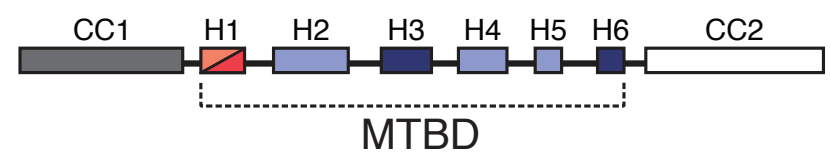

Figure 2.5. Molecular Dynamics study of the dynein MTBD conformations bound to MTs. (A) Crystal structures of the low-affinity conformation (PDB 3ERR) and tubulin (PDB 1JFF) rigid-body docked into our cryo-EM density map. H1 is shown in orange and displays steric clashes with the MT. Adjacent to $\mathrm{H} 1$ is an unoccupied portion of the map (black arrow). (B) Pseudo-atomic model of the low-affinity conformation bound to MTs obtained after $15 \mathrm{~ns}$ of MD simulations. H1 moves to a position that avoids clashes with the MT. (C) Pseudo-atomic model of a putative intermediate state obtained after molecular dynamics flexible fitting (MDFF). The repositioning of $\mathrm{H} 1$ and $\mathrm{H} 3$ is achieved but the coiled-coil registry is still in the low-affinity conformation. (D) Pseudo-atomic model of the dynein MTBD in its high-affinity state obtained after MDFF and targeted molecular dynamics (TMD). The coordinates of the $\mathrm{C} \alpha$ atoms corresponding to a shift of half heptad (4 aa) were used as a target position to guide the registry shift. The crosscorrelation coefficient (CCC) between the target density map and each structure at $9.7 \AA$ resolution is indicated below each panel. Color-coding is indicated for each set of structures. 
registry would make it protrude from the map, incurring a penalty in the simulations. We achieved the shift by applying targeted molecular dynamics (TMD) (37) to the tip of the coiledcoil (Figure 2.5 D), using the $\mathrm{C} \alpha$ coordinates of the half-heptad shift in our construct to guide the final position of the stalk during the simulations. This final model (Figure 2.4 B, D), which we refer to as the high-affinity MTBD, has the highest cross-correlation with the experimental map (Figures 2.5 and 2.6).

We repeated the MDFF calculations using the MTBD from the recent crystal structure of an ADP-bound dynein monomer (14) (Figure 2.7). The only difference in the resulting pseudoatomic model is in the stalk, where the dynein monomer's structure is missing density for one of the helices next to the MTBD (Figure 2.7 A, B). The similarity in the crystal structures of the MTBD in the low-affinity and ADP-bound states is likely due to the absence of MTs; our results suggest that the conformation we observe in our MT-bound high-affinity structure is stabilized by its interactions with $\beta$-tubulin (Figure $2.8 \mathrm{~A}$, Tables 1-1 and 1-2). MDFF of the ADP-bound dynein monomer's MTBD into our cryo-EM map results in a large change in the angle between the MTBD and the stalk in the dynein monomer structure (Figure 2.7 A, B). This change makes the docking of the dynein monomer into our map compatible with previously reported measurements of the MT-stalk angle $(17,20)$ and our two-dimensional analysis of images of monomer-decorated MTs (Figure 2.7 D, E).

The cryo-EM map shows three points of density between the MT and dynein's MTBD: the H1-H2 loop and helices H3 and H6 (Figure 2.4). Several parts of the structure are unchanged by its interaction with the MT, especially helices $\mathrm{H} 6, \mathrm{H} 5$, and $\mathrm{CC}$, with root mean square deviations (RMSD) between the low- and high-affinity models of $1.4 \AA, 1.4 \AA$, and $1.8 \AA$, 


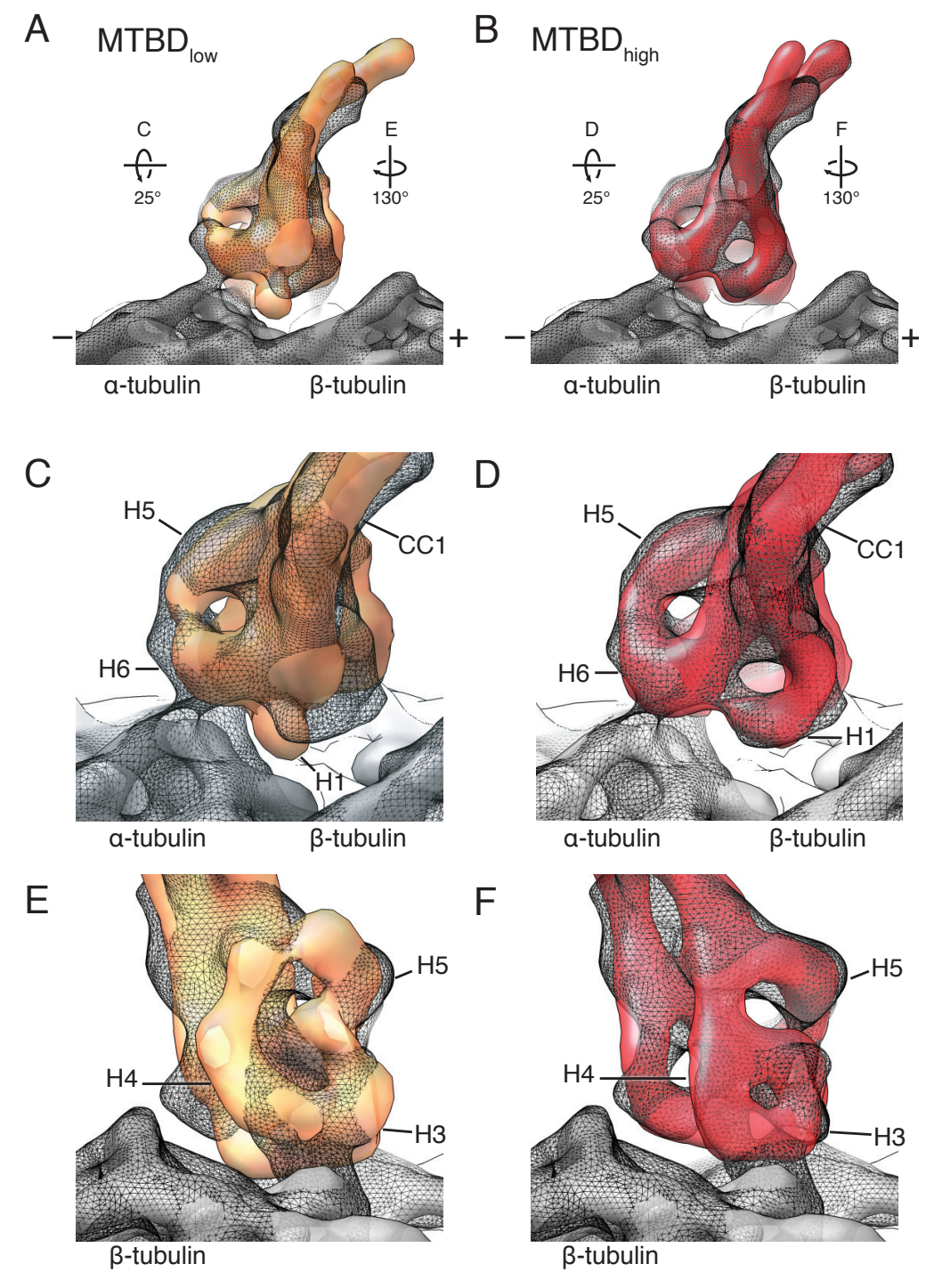

Figure 2.6. Comparison of $10 \AA$ synthetic maps of the low- and high-affinity MTBD conformations with our experimental map. The stalk and MTBD of the low-affinity conformation (PDB 3ERR) and the pseudo-atomic model of the high-affinity conformation of the MTBD were converted to synthetic EM maps, filtered to a resolution of $10 \AA$, and oriented relative to the cryo-EM map identically to the atomic models shown in Fig. 2. (A) $10 \AA$ synthetic map of the low-affinity conformation $\left(\mathrm{MTBD}_{\text {low }}\right.$, orange) docked into the cryo-EM map (grey mesh). (C, E) Close-ups of the structure shown in (A); the direction of each view is indicated in panel (A). (B) $10 \AA$ synthetic map of the pseudo-atomic model of the high-affinity conformation $\left(\mathrm{MTBD}_{\text {high, }}\right.$ red) docked into the cryo-EM map. (D, F) Close-ups of the structure shown in (B); the direction of each view is indicated in panel (B). The synthetic map of the lowaffinity conformation protrudes from our experimental map at several locations $(\mathrm{CCC}=0.648$ ), including $\mathrm{H} 1$ (C) and H4 (E). In contrast, the synthetic map of the pseudo-atomic model exhibits a better overall fit to our experimental map $(\mathrm{CCC}=0.751)$. 
Figure 2.7. Different atomic resolution structures of the MTBD converge on a single MT-bound conformation. (A) The MTBD from a recently reported atomic resolution structure of ADP-

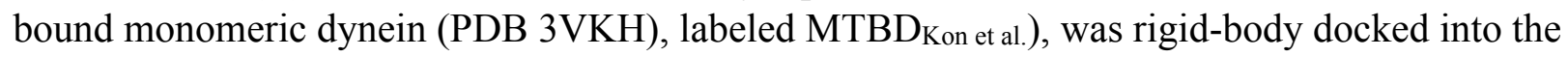
experimental map. Note that helices H1 (orange) and CC1 (grey) lie outside of the experimental

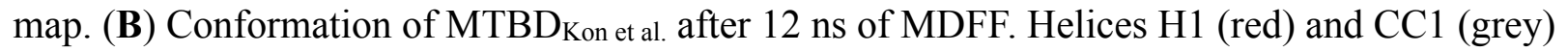
have moved into our experimental map. The conformation of the MTBD used in this study

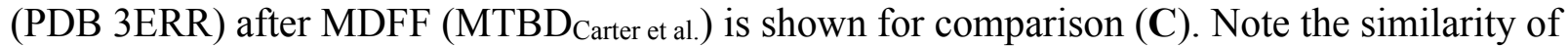
the MDFF models in (B) and (C). (D) The atomic resolution structure of ADP-bound monomeric dynein, shown in red, was rigid body docked into a section of one protofilament (PF1) of the experimental map. The orientation of the MTBD is shown in (A). Tubulin is colored green. The resulting conformation of the entire monomer results in clashes with the neighboring protofilament (PF2). A second monomer model (blue) was obtained by replacing the MTBD (residues 3352 to 3491) from the crystal structure with the MDFF-generated $\mathrm{MTBD}_{\mathrm{Kon}}$ et al. shown in (B) (See material and methods for details). The monomer containing the MDFF-generated $\mathrm{MTBD}_{\mathrm{Kon}}$ et al. exhibits no clashes with our experimental map, and its overall configuration closely matches class averages of $S$. cerevisiae monomeric dynein bound to MTs (E). For panels (D) and (E) the approximate angle of the stalk relative to the MT $\left(\sim 60^{\circ}\right)$ is indicated. For all panels MT polarity is indicated.. 
Figure 2.7 (Continued)

A

Rigid body docking with $\mathrm{MTBD}_{\text {Kon et al. }}$
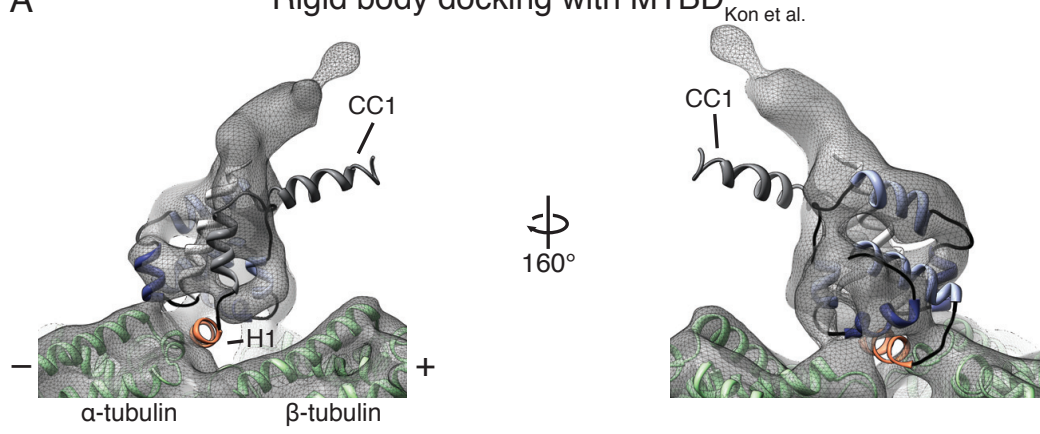

B

MDFF with $\mathrm{MTBD}_{\text {Kon et al. }}$
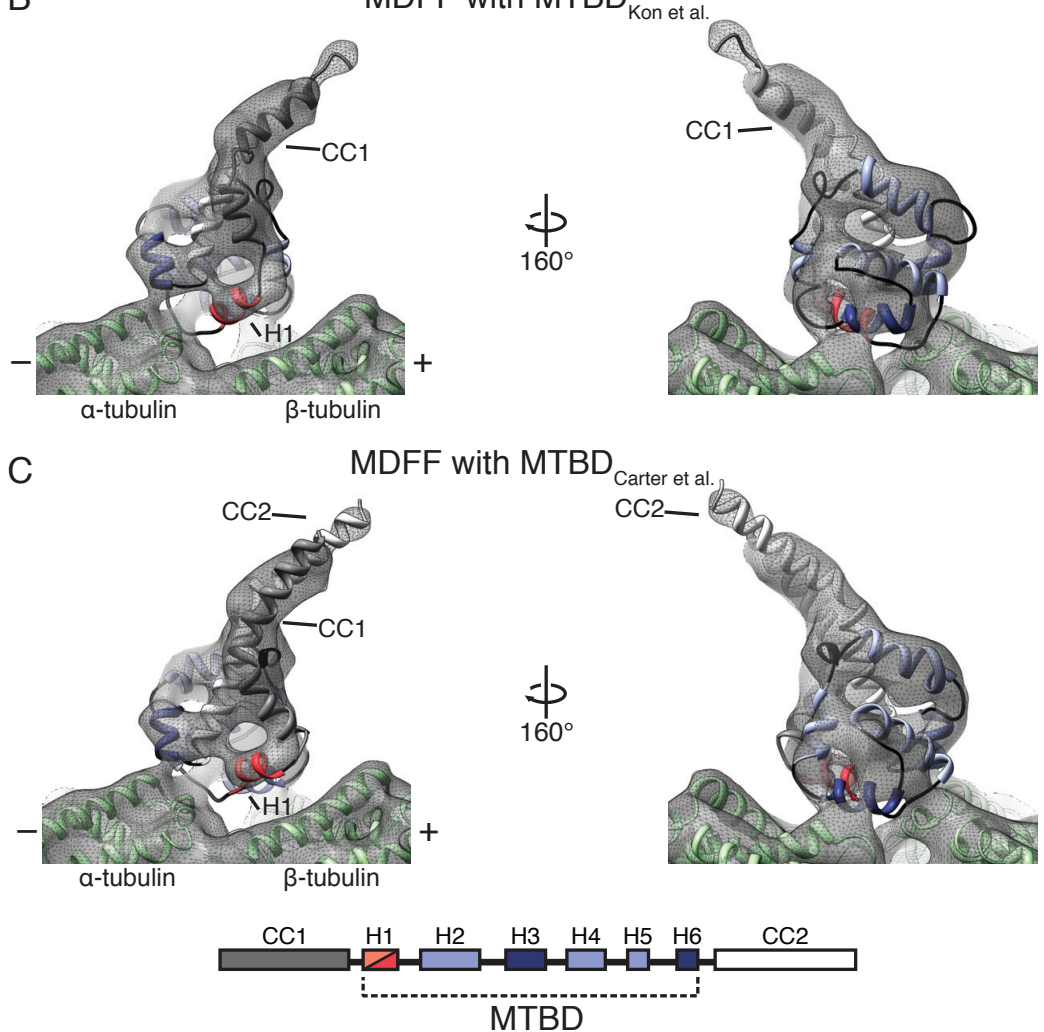

D

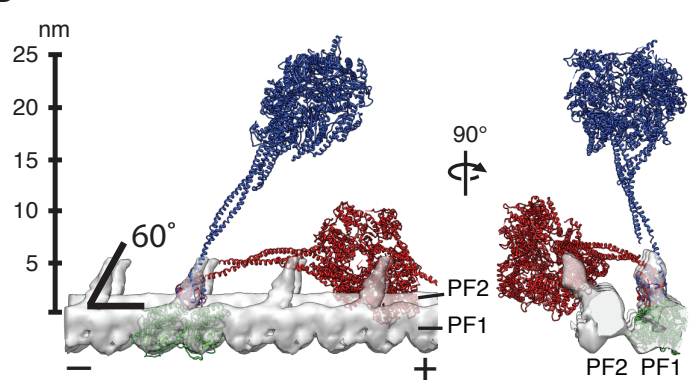

E

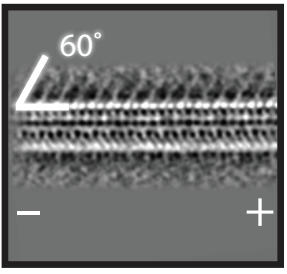

Class average

Kon et al. monomer with MTBD, rigid body docked

Kon et al. monomer aligned to MDFF model from (B) 
respectively. The largest changes are the repositioning of H1 (RMSD $=10.1 \AA)$ and an opening of $\mathrm{CC} 1$ in the coiled-coil next to the MTBD (RMSD = 8.1 $\AA$ ) (Figure 2.3 A-D), a movement anchored at the proline kink present in $\mathrm{CC} 1$. The final position of $\mathrm{H} 1$ is stabilized by multiple interactions with an acidic patch in $\mathrm{H} 12$ of $\beta$-tubulin not fully occupied in the low-affinity state (Figure $2.8 \mathrm{~A}$ ). This patch also stabilizes the high-affinity state of kinesin (44).

We monitored hydrogen bonds and salt bridges formed between the MTBD and the MT during MD simulations (Tables 1-2 and 1-3); the high-affinity MTBD formed more hydrogen bonds with the MT (Figure 2.9) and electrostatic interactions at H1, H3, and H6 (Figure 2.8, Table 1-2). Nearly all of these residues are highly conserved, and mutating them results in defects in MT binding $(18,45)$ (Figure 2.8). The importance of salt bridges to the MTBD-MT interaction is consistent with the sensitivity of dynein's motility to ionic strength (Figure 2.10).

Our structural analysis suggested that the MTBD contains residues that lower its own affinity for the MT. In the MD simulations, basic residues in $\mathrm{H} 1$ and $\mathrm{H} 6$ formed salt bridges that alternated between intramolecular and intermolecular partners. In the MT-bound high-affinity conformation, H1-K3298 switched between a glutamate on $\beta$-tubulin and a conserved glutamate in CC1 (E3289) (Figures 2.11 A, 2.12 A); neither contact can be formed by H1-K3298 in the low-affinity conformation (Figure 2.8 A). H6-R3382 switched from an intramolecular interaction with a conserved glutamate in the same helix (H6-E3378) in the low-affinity unbound state to an intermolecular interaction with a cluster of glutamates on $\alpha$-tubulin upon binding (Figure 2.11 B, $2.12 \mathrm{~B}$ ); the intramolecular interaction might weaken the MTBD-MT interaction in both the lowand high-affinity conformations. The importance of the two MTBD glutamates involved in the intramolecular salt bridges had previously been recognized; substitution of CC1-E3289 and H6- 
Figure 2.8. Conserved intermolecular MTBD-MT and intramolecular MTBD interactions in the dynein high-affinity state. (A) Top: Salt bridges formed between the MTBD and the MT, as detected by MD simulations in the high-affinity pseudo-atomic model, are labelled within the indicated helices of the MTBD. Bottom: The corresponding views for the MT-bound lowaffinity state are shown. Tubulin is colored green. (B) Alignment of the MTBD from diverse cytoplasmic dynein sequences calculated with MAFFT and visualized with Jalview according to the Clustal coloring scheme for residues with identity $\geq 50 \%$. Of interest to this study, basic and acidic residues are colored red and purple, respectively. Residues that are conserved between Dictyostelium discoideum ( $D d$ and Mus musculus $(\mathrm{Mm})$ and form salt bridges with MT residues are labeled and annotated according to the legend above. One $M m$ residue, R3342, forms a salt bridge with the MT and is not present in Dd dynein. Intramolecular MTBD salt bridges (E3289K3298 and E3378-R3382 (Figure 2.11) are connected with a dashed line, as is an intramolecular interaction between P3311 and W3333, which is maintained in both high- and low-affinity states. 
Figure 2.8 (Continued)
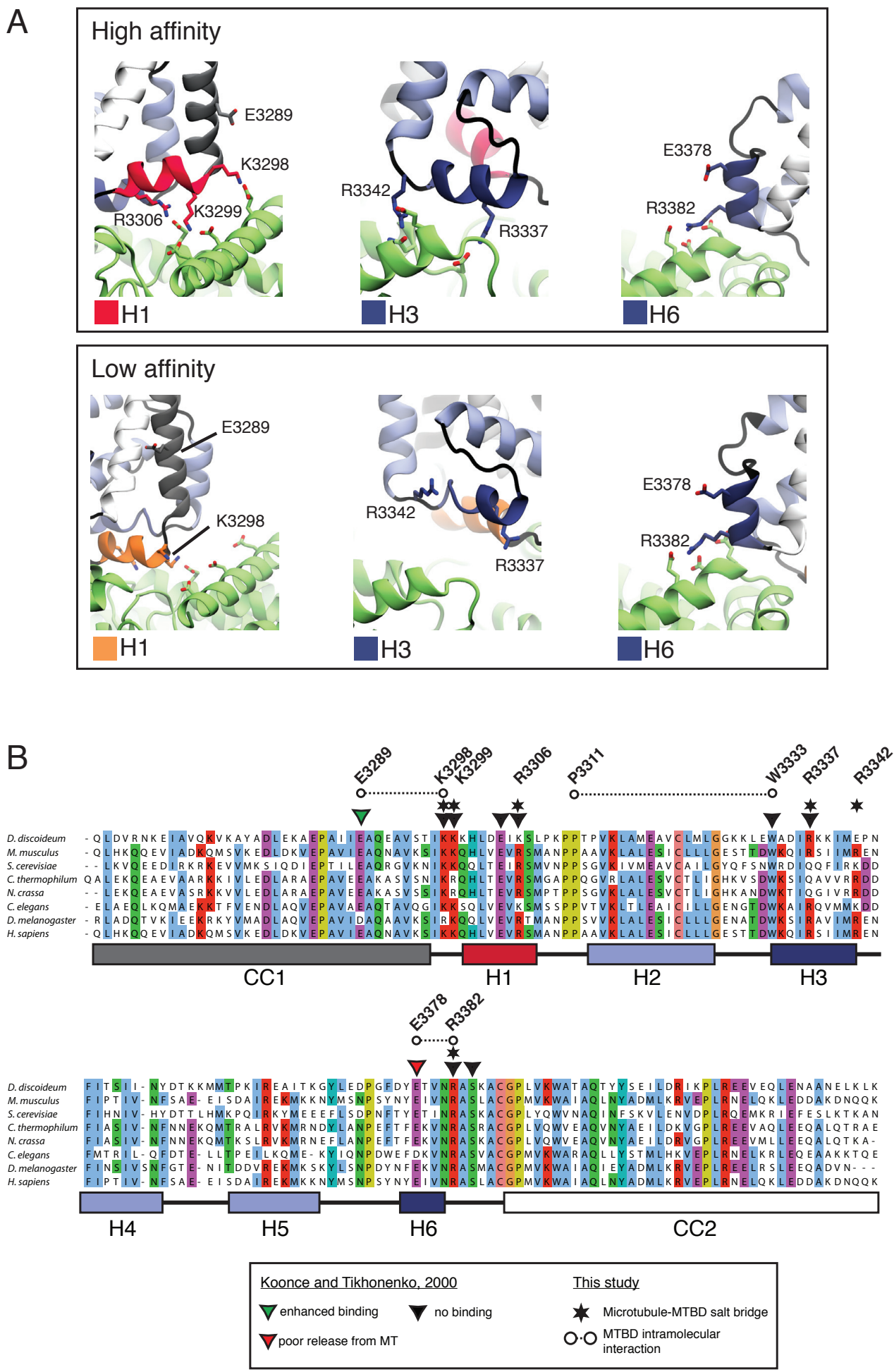
Table 2-2. Prominent interactions involving the high-affinity state of dynein's MTBD. Percentages calculated from last $10 \mathrm{~ns}$ of MD simulations. Interactions listed were observed for at least $1 \mathrm{~ns}$ along the $10 \mathrm{~ns}$ trajectory. Omitted were intramolecular hydrogen bonds that form the alpha helices of the dynein's MTBD. * Calculated from 15 ns of MDFF+TMD simulations.

\begin{tabular}{|c|c|}
\hline \multicolumn{2}{|c|}{ High Affinity State Intermolecular Interactions } \\
\hline MTBD-tubulin Interaction & occupancy \\
\hline $\mathrm{R} 3382-\alpha \mathrm{E} 417$ & $23 \%$ \\
\hline R3382- $\alpha E 420$ & $79 \%$ \\
\hline R3382- $\alpha \mathrm{G} 416$ & $40 \%$ \\
\hline K3299- $\beta E 431$ & $60 \%$ \\
\hline K3299- $\beta$ D 427 & $53 \%$ \\
\hline R3342- $\beta$ E159 & $51 \%$ \\
\hline $\mathrm{N} 3310-\alpha \mathrm{G} 410$ & $38 \%$ \\
\hline L3385- $\alpha \mathrm{E} 415$ & $48 \%$ \\
\hline S3384- $\alpha \mathrm{G} 415$ & $27 \%$ \\
\hline A3383- $\alpha \mathrm{E} 415$ & $49 \%$ \\
\hline R3306- $\beta$ E196 & $86 \% *$ \\
\hline 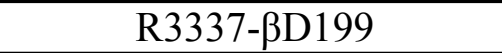 & $2 \% *$ \\
\hline R3342-ßN197 & $36 \% *$ \\
\hline \multicolumn{2}{|c|}{ High Affinity State Intramolecular Interactions } \\
\hline MTBD Residues & occupancy \\
\hline K3366-D3402 & $52 \%$ \\
\hline K3405-E3408 & $40 \%$ \\
\hline Y3369-E3320 & $65 \%$ \\
\hline H3301-E3304 & $62 \%$ \\
\hline R3362-D3359 & $61 \%$ \\
\hline R3406-D3359 & $65 \%$ \\
\hline Q3397-S3353 & $38 \%$ \\
\hline R3306-E3343 & $19 \%$ \\
\hline K3427-D3423 & $20 \%$ \\
\hline R3406-D3402 & $37 \%$ \\
\hline K3282-E3278 & $11 \%$ \\
\hline T3330-Q3335 & $16 \%$ \\
\hline K3416-D3420 & $11 \%$ \\
\hline K3264-E3267 & $22 \%$ \\
\hline Y3400-E3284 & $34 \%$ \\
\hline K3416-E3413 & $12 \%$ \\
\hline T3331-E3320 & $11 \%$ \\
\hline
\end{tabular}


Table 2-3. Prominent interactions involving the low-affinity state of dynein's MTBD. Percentages calculated from last $10 \mathrm{~ns}$ of MD simulations. Interactions listed were observed for at least $1 \mathrm{~ns}$ along the $10 \mathrm{~ns}$ trajectory. Omitted were intramolecular hydrogen bonds that form the alpha helices of the dynein's MTBD.

\begin{tabular}{|c|c|}
\hline \multicolumn{2}{|c|}{ Low Affinity State Intermolecular Interactions } \\
\hline MTBD-tubulin Interaction & occupancy \\
\hline A3383- $\alpha$ E415 & $45 \%$ \\
\hline $\mathrm{R} 3382-\alpha \mathrm{G} 416$ & $44 \%$ \\
\hline R3382- $\alpha \mathrm{E} 417$ & $61 \%$ \\
\hline R3382- $\alpha \mathrm{E} 420$ & $79 \%$ \\
\hline K3299- $\beta$ E196 & $27 \%$ \\
\hline S3307-ßR253 & $16 \%$ \\
\hline $\mathrm{L} 3385-\alpha \mathrm{E} 415$ & $34 \%$ \\
\hline $\mathrm{N} 3310-\alpha \mathrm{K} 112$ & $10 \%$ \\
\hline \multicolumn{2}{|c|}{ Low Affinity State Intramolecular Interactions } \\
\hline MTBD Residues & occupancy \\
\hline K3366-D3402 & $61 \%$ \\
\hline R3366-E3363 & $43 \%$ \\
\hline R3342-E3328 & $87 \%$ \\
\hline R3411-E3355 & $51 \%$ \\
\hline S3353-E3356 & $15 \%$ \\
\hline K3405-E3408 & $52 \%$ \\
\hline Y3369-E3320 & $45 \%$ \\
\hline H3301-E3304 & $37 \%$ \\
\hline K3392-E3289 & $57 \%$ \\
\hline K3316-E3320 & $23 \%$ \\
\hline S3321-Y3375 & $43 \%$ \\
\hline R3362-D3359 & $59 \%$ \\
\hline K3272-E3413 & $54 \%$ \\
\hline S3374-N3372 & $15 \%$ \\
\hline T3331-E3320 & $29 \%$ \\
\hline K3366-E3363 & $38 \%$ \\
\hline K3282-D3279 & $29 \%$ \\
\hline R3406-D3402 & $20 \%$ \\
\hline
\end{tabular}



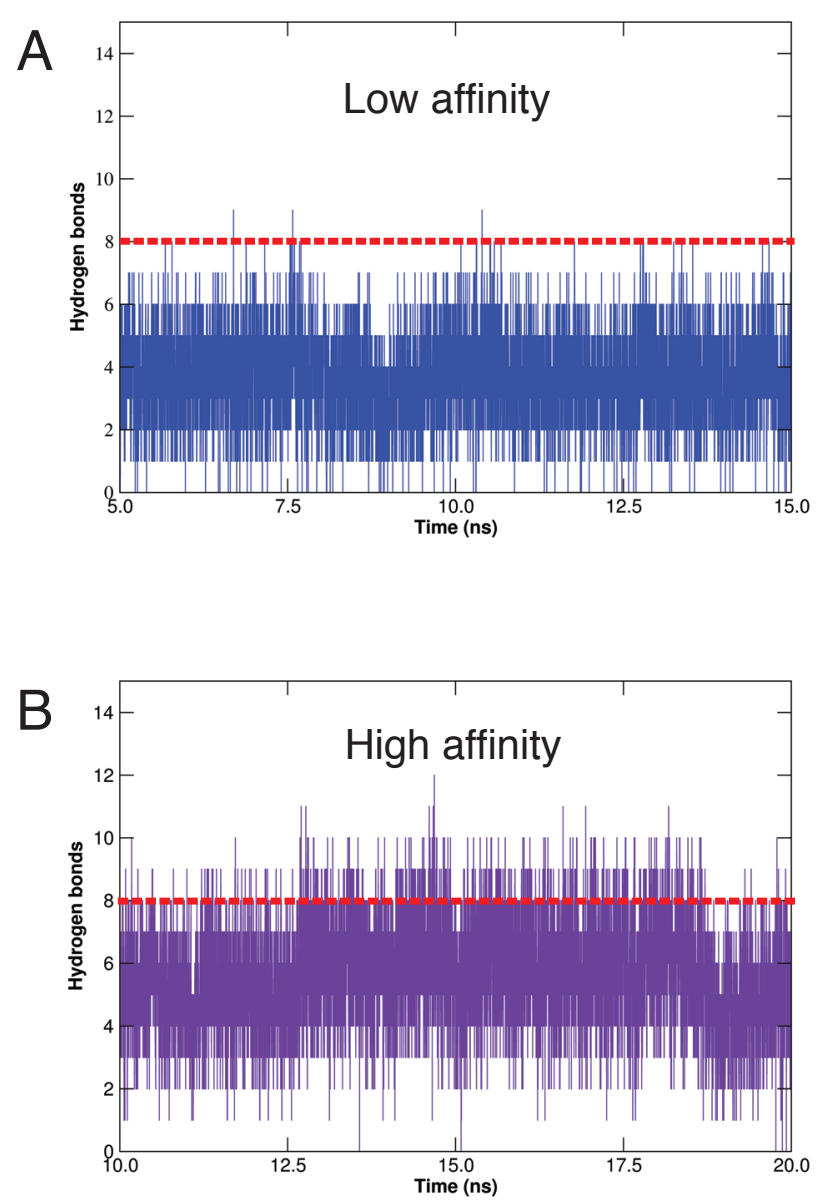

Figure 2.9. Formation of hydrogen bonds between the dynein MTBD and tubulin during MD simulations. Hydrogen bonds formed between the dynein MTBD and tubulin over time are shown for the (A) dynein low-affinity state and the (B) dynein high-affinity state. Hydrogen bonds were counted for the last $10 \mathrm{~ns}$ of the final MD equilibration simulations when the distance between the donor and acceptor was within $3.0 \AA$ and the angle between donor and hydrogen-acceptor was greater than $160^{\circ}$. A dashed line marks the same point on the $y$-axis in both graphs. 


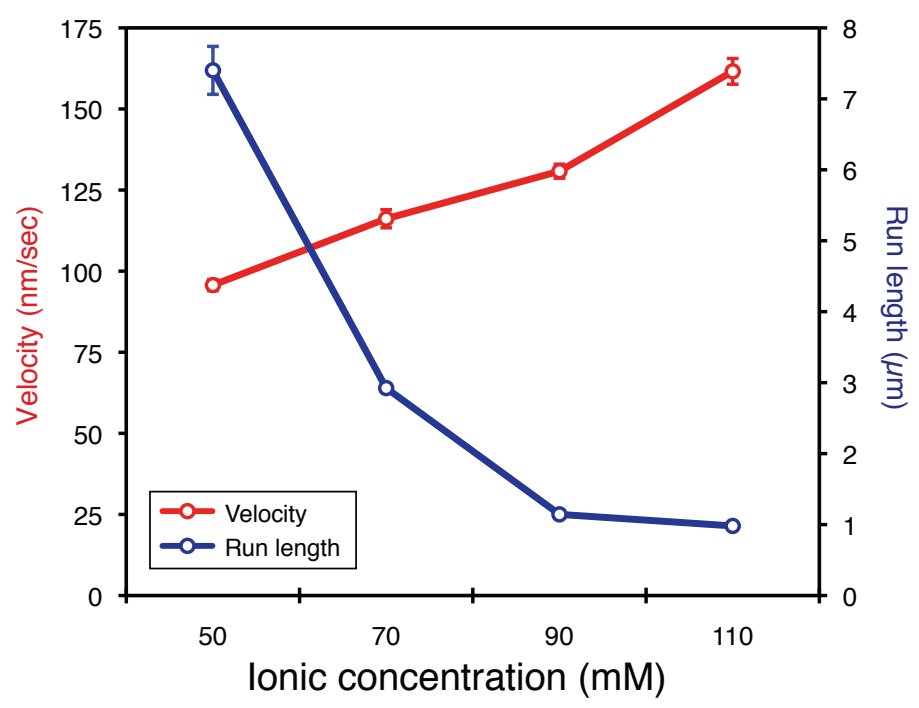

Figure 2.10. Effect of increasing ionic strength on dynein motility. Fluorescently labeled dynein motors were tracked on axonemes by total internal reflection (TIRF) microscopy. The run length and velocity were determined in assay buffer supplemented with increasing concentrations of potassium acetate (KAc). Total ionic concentration is the sum of added KAc and a constant concentration of buffering agent. The run length at each point is the mean \pm standard deviation. The velocity at each point is the mean \pm standard error. The average length of analyzed axonemes (mean \pm standard deviation) was $24.5 \pm 8.0 \mu \mathrm{m}$. 


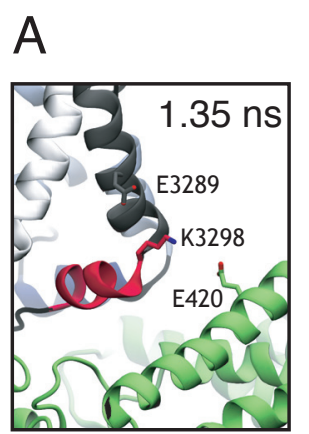

Intermediate

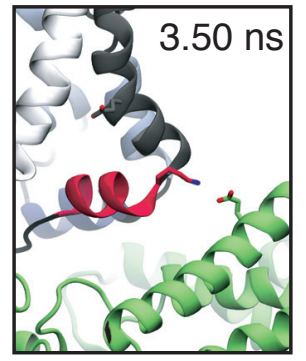

MTBD-MT

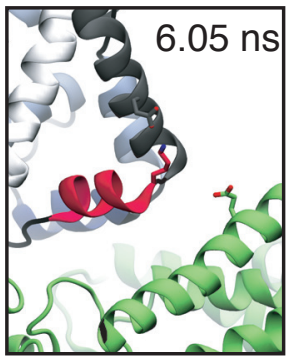

Intra-MTBD

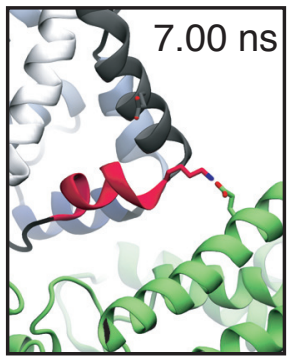

MTBD-MT

$\mathrm{B}$

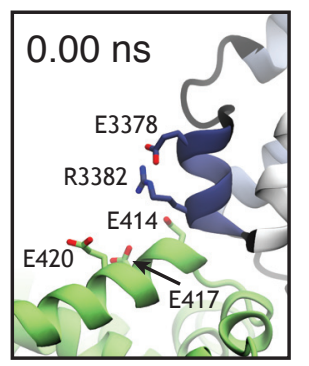

Intra-MTBD

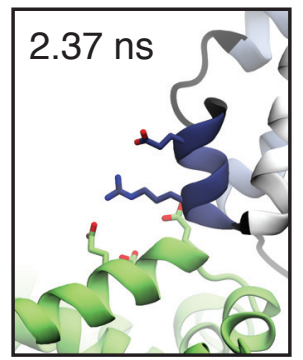

Intermediate

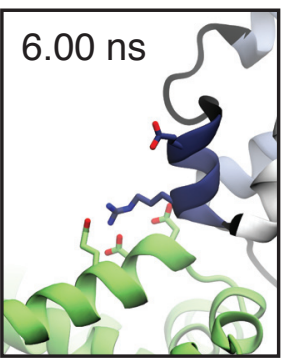

MTBD-MT

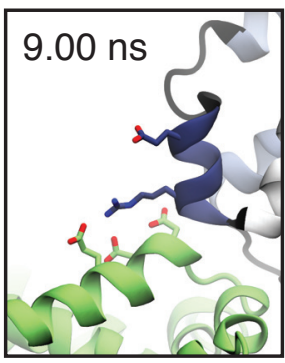

CC2

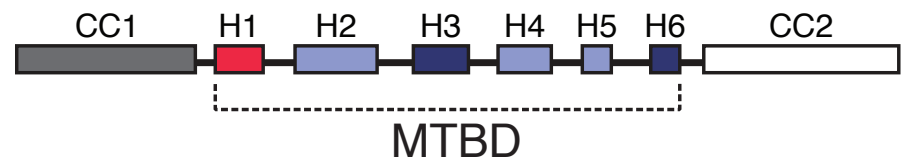

Figure 2.11. Behavior of dynamic salt bridges in the MTBD as determined by MD. (A) K3298 in $\mathrm{H} 1$ of the MTBD alternates between an intermolecular salt bridge with E420 on $\beta$-tubulin (MTBD-MT) and an intramolecular salt bridge with E3289 on CC1 of the MTBD (intraMTBD). (B) R3382 in H6 of the MTBD alternates between an intermolecular salt bridge with E414 and E420 on $\alpha$-tubulin (MTBD-MT) and an intramolecular salt bridge with E3378 in H6 (intra-MTBD). Single letter amino acid code and number are indicated for Bos taurus tubulin and Mus musculus cytoplasmic dynein. Time stamps for frames from MD simulations are indicated. Intermediate refers to a position midway between MTBD-MT and intra-MTBD salt bridges. 

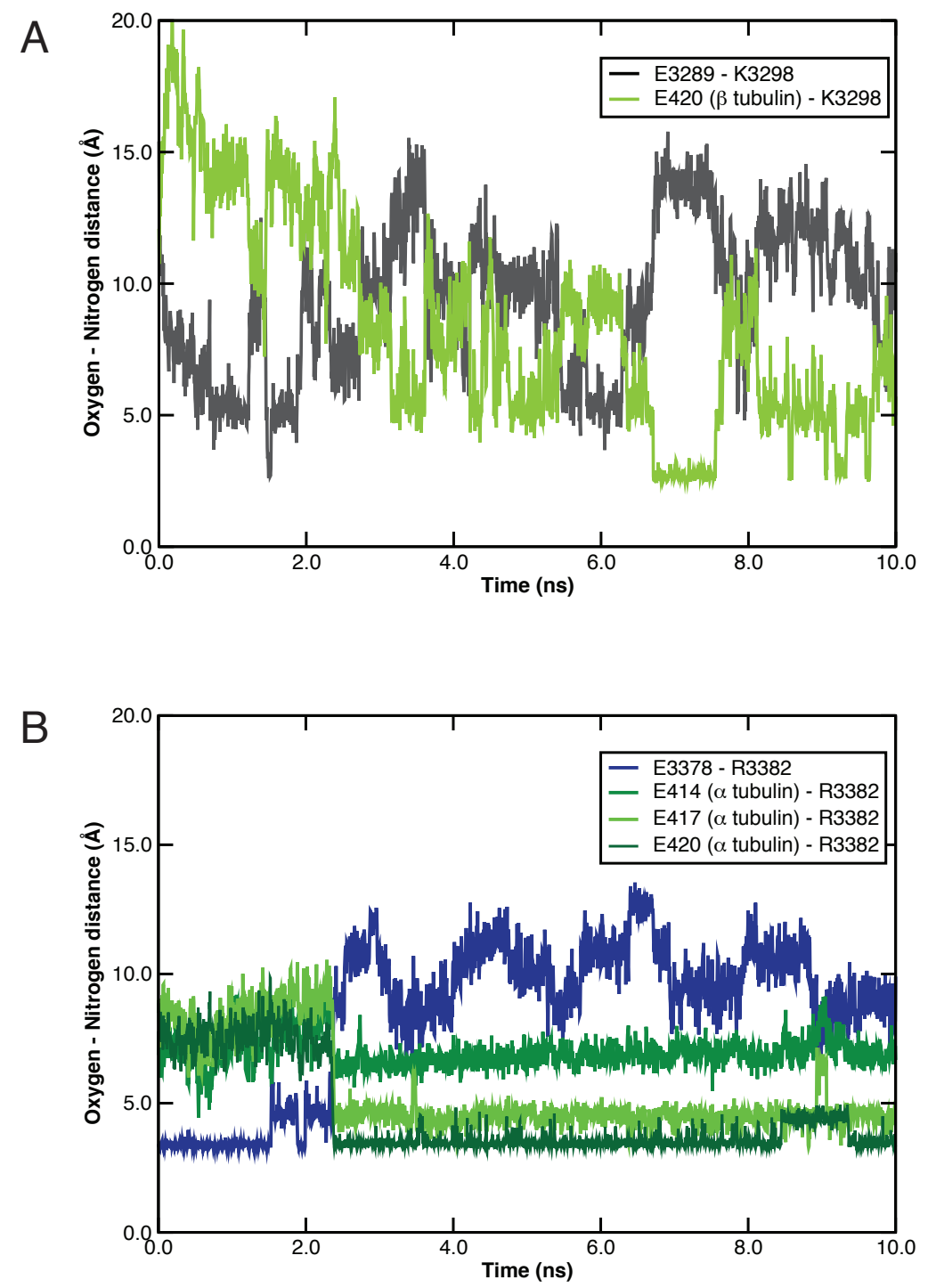

Figure 2.12. Dynamic salt bridges in $\mathrm{H} 1$ and $\mathrm{H} 6$ of the MTBD as observed by MD simulations. (A) Dynamic salt bridges at H1 are formed between K3298 and either E3298 in the dynein MTBD or E420 in $\beta$-tubulin. (B) Dynamic salt bridges at H6 are formed between R3382 and either E3378 in the dynein MTBD or E414, E417 and E420 in $\alpha$-tubulin. For both (A) and (B) the average oxygen-nitrogen distances over time for the indicated salt bridges are shown. 
E3378 with alanine increased dynein's MT-binding affinity $(18,45)$ and reduced its ATPstimulated release from MTs, respectively (45). We hypothesized that these phenotypes resulted from the competition between MT and MTBD residues in $\mathrm{CC} 1$ and $\mathrm{H} 6$ for salt bridge formation with the basic residues K3298 and R3382 in the MTBD.

To test this prediction we mutated the residues equivalent to E3289 and E3378 in CC1 and H6 of Saccharomyces cerevisiae dynein to either an isosteric but neutral (Q) or a basic amino acid $(\mathrm{K})$ to disrupt the salt bridge $(\mathrm{Q})$ or introduce an intramolecular charge repulsion $(\mathrm{K})$ that may favor intermolecular interactions between H1-K3298 or H6-R3382 and acidic residues on the MT surface. Single molecule motility assays that monitored the movement of purified mutant dyneins showed significant increases in dynein's run length and small decreases in velocity that paralleled the severity of the mutation (E -> Q -> K) (Figure 2.13 A, B and Figure 2.14). Most dramatically, the basic substitutions CC1-E3289K and H6-E3378K increased dynein's run length by five-fold and six-fold, respectively (Figure $2.13 \mathrm{~B}$ ), and the double mutant even further (Figure 2.15). These results suggest that cytoplasmic dynein has been selected for sub-maximal processivity. The effects observed with the mutants are not due to a strengthened interaction with the unstructured carboxyl-terminal tails of tubulin (E-hooks). Although their removal decreased the run length of all constructs tested, in agreement with previous studies (46), the trend of increasing processivity (WT -> E3289K -> E3378K) remained (Figure 2.16).

These findings provide a molecular model for how dynein couples its affinity for MTs with the nucleotide state of the motor domain (Figure 2.13 C-E). We describe the transition from low to high affinity, but suggest that the proposed changes are reversible. During a diffusive search 

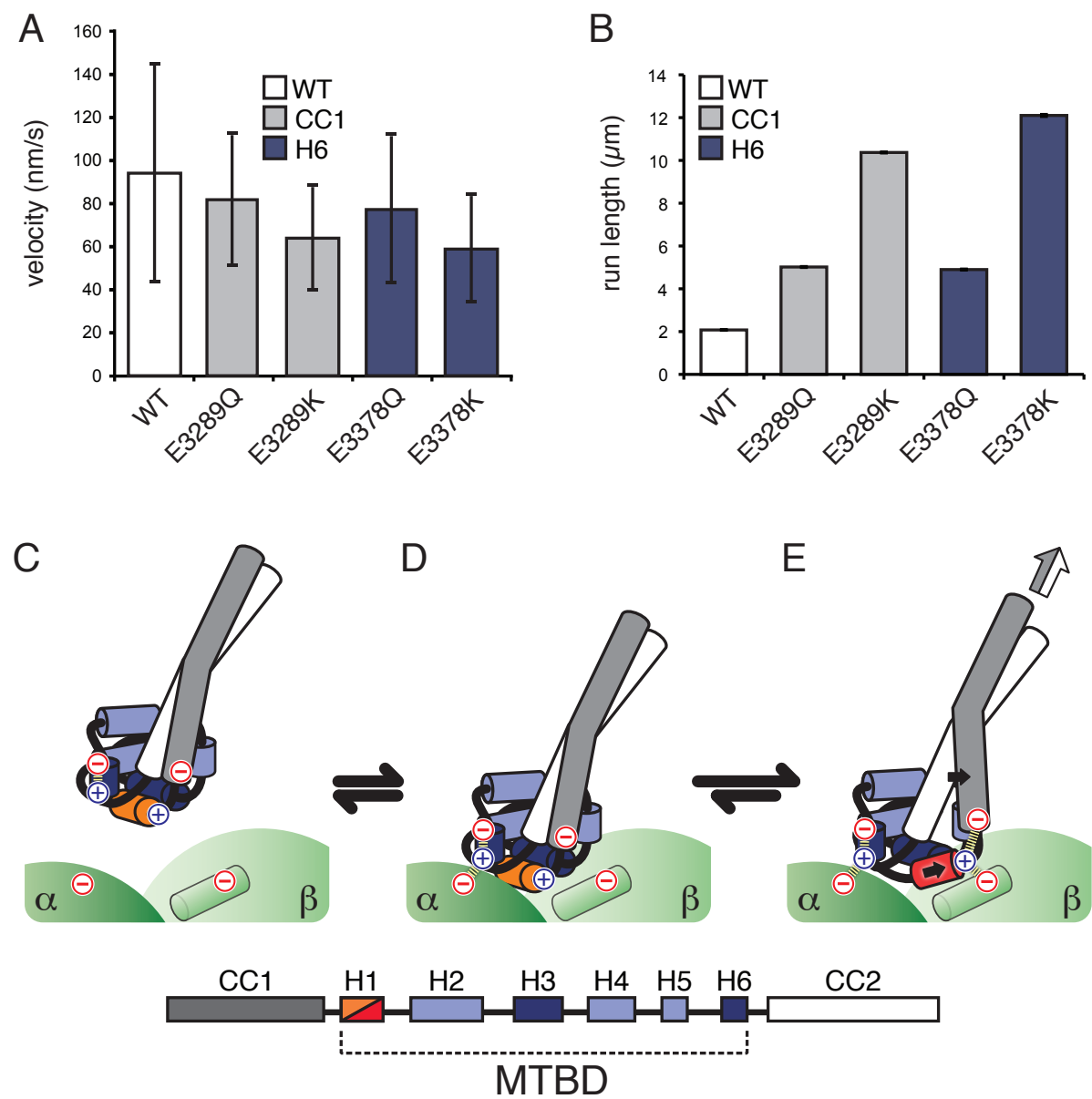

Figure 2.13. Dynamic salt bridges reduce dynein motility. Bar graphs of (A) mean velocities and (B) characteristic run lengths of fluorescently labeled Saccharomyces cerevisiae dynein bearing the equivalent of the indicated Mus musculus mutations moving on MTs. Error bars: standard deviation, SD (A) and standard error of the mean, SE (B). Velocity and run length differences between WT and Q mutants, as well as between Q and K mutations at the same position, are statistically significant ( $\mathrm{t}$-test, $\mathrm{P}<0.01$ for velocity, and two-tailed KS-test, $\mathrm{P}<$ 0.01 for run length). The data for the double mutant (E->K at both $\mathrm{CC} 1$ and H6) was omitted because run lengths could only be determined under more stringent motility conditions (Figure 2.15). (C-E) Molecular model for the coordination of nucleotide state and MT binding by dynein (see text for details). (C) Unbound dynein in the low affinity conformation, $\mathrm{H} 1$ is colored orange. (D) Initial interaction with a new binding site. (E) Repositioning of H1 (now in red) leads to the formation of new ionic interactions with $\beta$-tubulin (green cylinder) that stabilize the high-affinity state of the MTBD. The repositioning of $\mathrm{H} 1$ is accompanied by a movement in $\mathrm{CC} 1$; both movements are indicated by solid black arrows. The conformational change in the MTBD biases the registry of the coiled-coil towards the high-affinity $\alpha$ state, a change that can propagate to the motor domain (white/grey arrow). Ionic interactions are indicated with dashed lines, The identities of the helices in the MTBD are indicated by the key. 
Figure 2.14. Dynamic salt bridges temper dynein motility. Data corresponds to that reported in Fig. 4. (A) Representative kymographs of fluorescently labeled dynein and dynein mutants moving along MTs. Each kymograph follows the trajectory of all dynein molecules along a stretch of a single MT. MT polarity is indicated and applies to all panels. Horizontal scale bar, 5 $\mu \mathrm{m}$; vertical scale bar, $1 \mathrm{~min}$. (B) Histograms of velocity and run length of WT dynein and dynein mutants from (A). All velocity data are plotted as mean +/- SD and all run length data are plotted as characteristic run length $+/-\mathrm{SE}, \mathrm{N}>300$. 
Figure 2.14 (Continued)
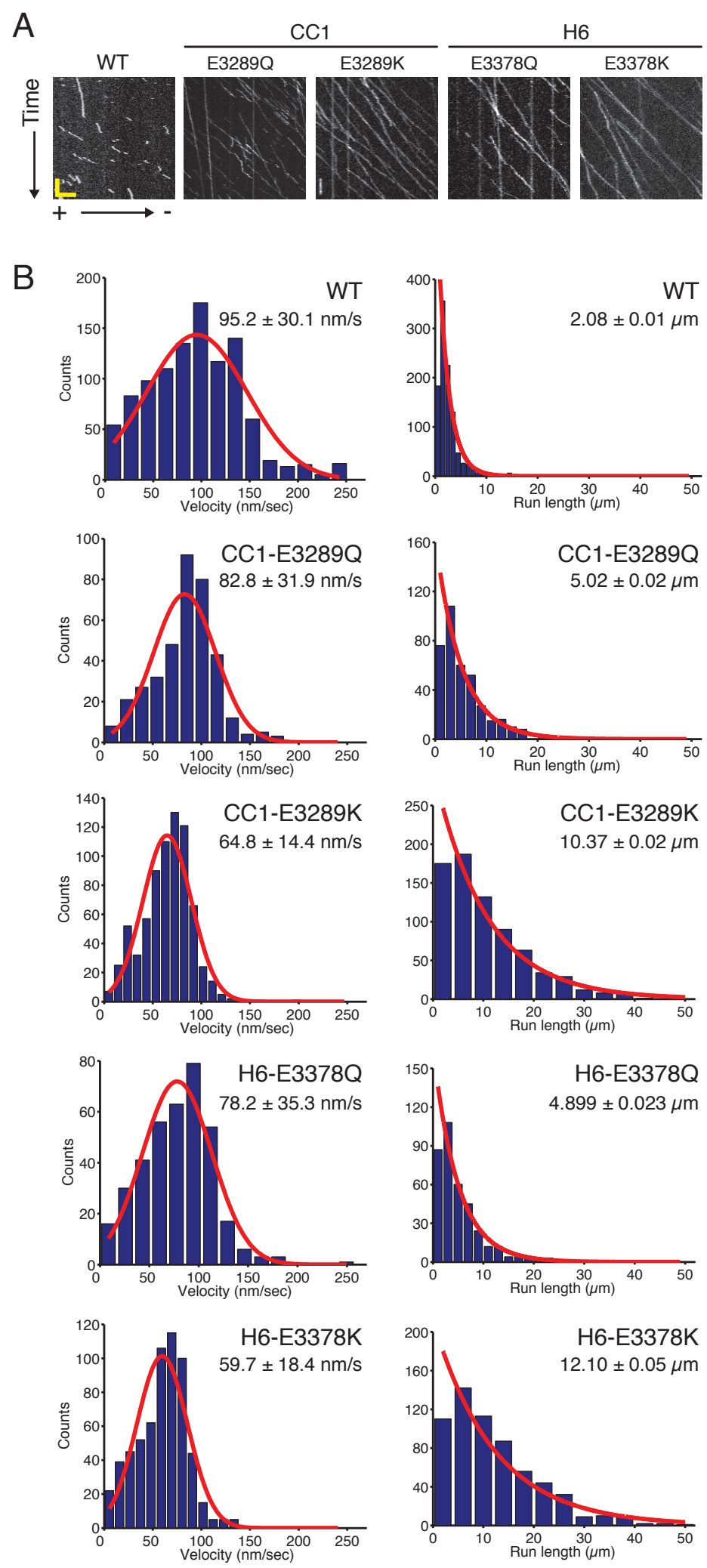
A

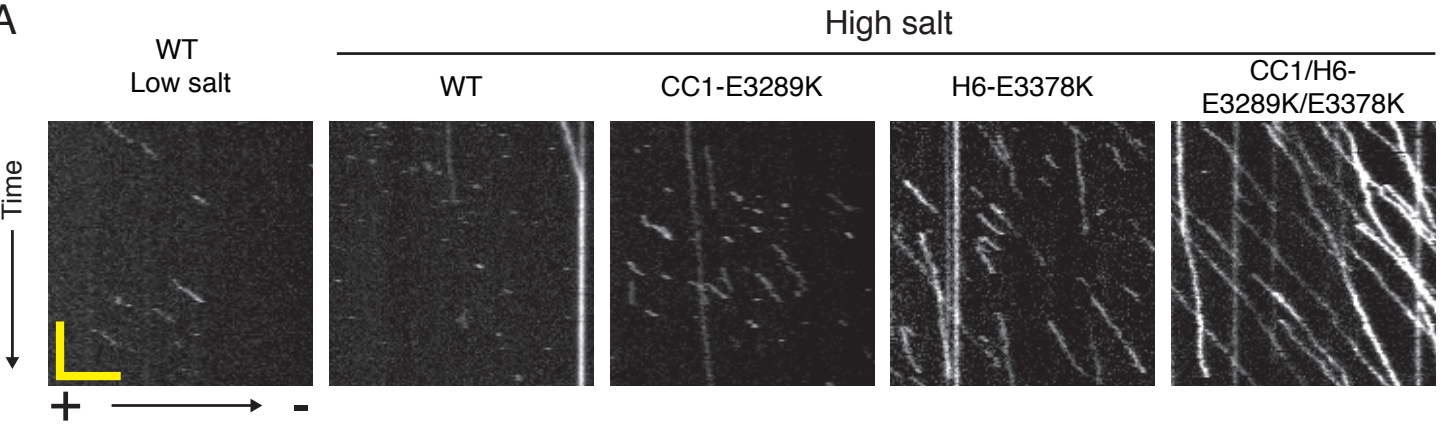

B

High salt
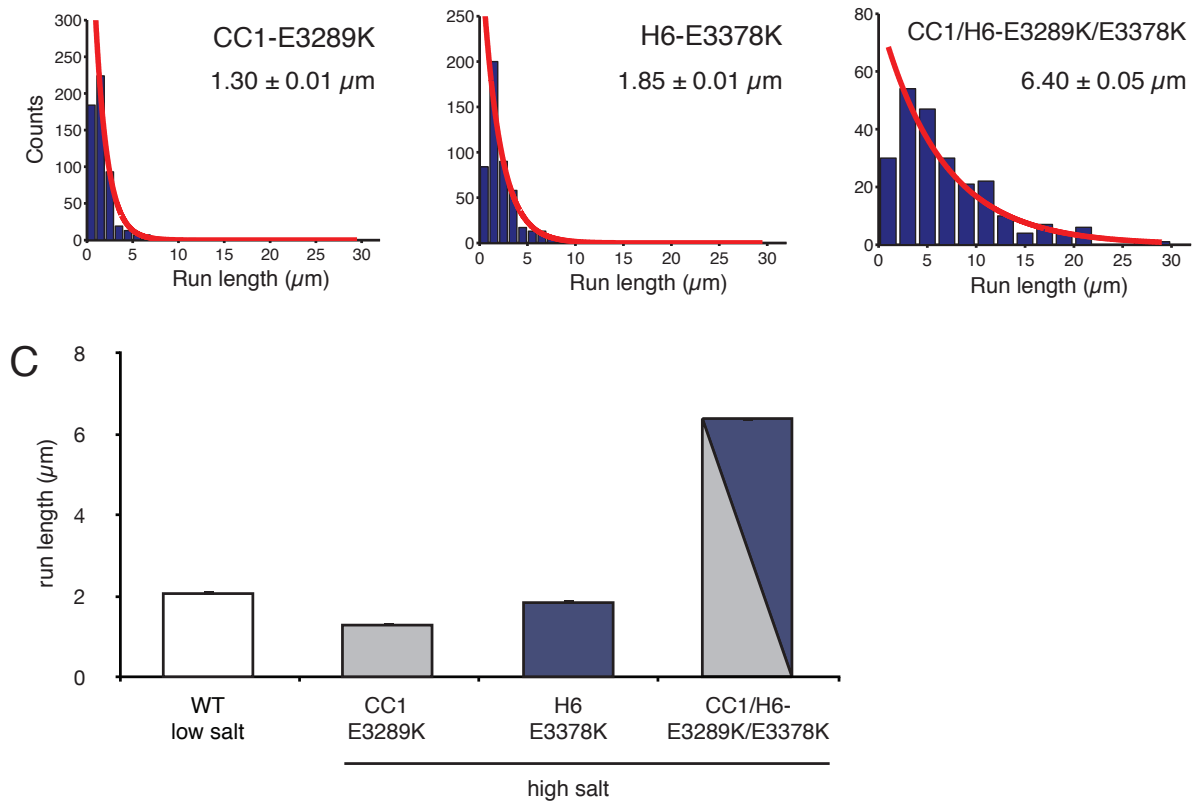

Figure 2.15. Dynamic salt bridge mutations increase dynein processivity under more stringent motility conditions. (A) Representative kymographs of fluorescently labeled dynein moving along MTs (low salt $=80 \mathrm{mM}$ total ionic strength, high salt $=130 \mathrm{mM}$ total ionic strength). Note that WT dynein motility is measurable only under low salt conditions, whereas CC1-E3289K and H6-E3378K dynein mutants exhibit run lengths in high salt that are similar to WT run lengths in low salt. The combined CC1/H6-E3289K/E3378K double mutant exhibits much longer run lengths. Horizontal scale bar, $5 \mu \mathrm{m}$; vertical scale bar, $1 \mathrm{~min}$. (B) Histograms of dynein run length corresponding to (A) are shown with their characteristic run length $\pm \mathrm{SE}$, $\mathrm{N}>250$. (C) Quantitation of data show in (A) and (B). The differences between CC1-E3289K and H6-E3378K, and H6-E3378K and CC1/H6-E3289K/E3378 are significant (two-tailed KStest, $\mathrm{P}<0.01$ ). 

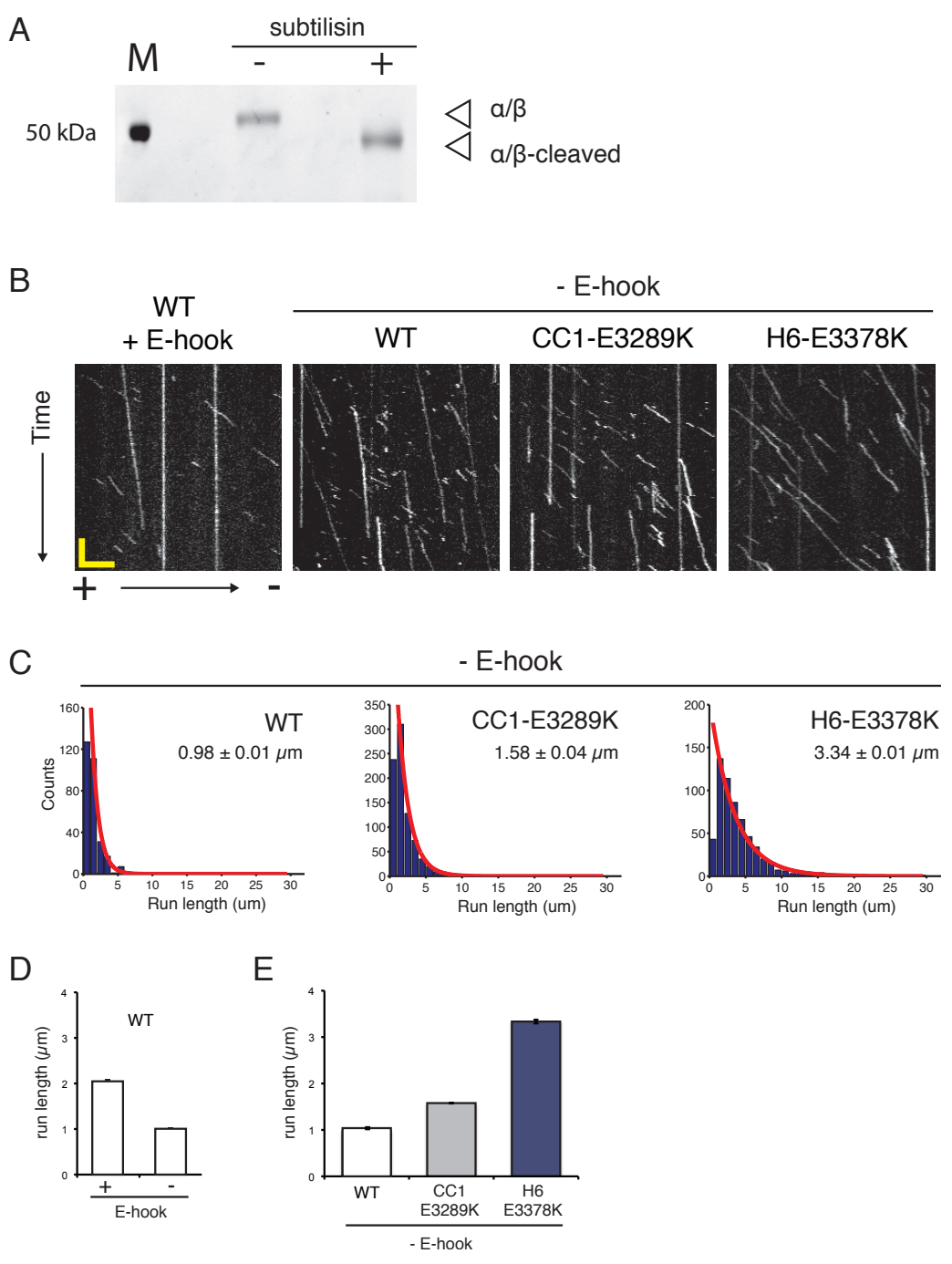

Figure 2.16. Dynamic salt bridge mutations increase dynein processivity independent of tubulin E-hooks. (A) SDS-PAGE analysis of tubulin used in single molecule motility assays.

Polymerized MTs were treated with subtilisin, which removes the C-terminal E-hook. Removal of the E-hook causes an increased mobility of $\alpha$ - and $\beta$ - tubulin on the gel. (B) Representative kymographs of fluorescently labeled dynein moving along MTs. The presence or absence of the E-hook is indicated. Note the visibly longer runs in CC1-E3289K and H6-E3378K relative to wild type dynein (WT). Horizontal scale bar, $5 \mu \mathrm{m}$; vertical scale bar, $1 \mathrm{~min}$. (C) Histograms of dynein run length corresponding to $(B)$ are shown with their characteristic run length $\pm \mathrm{SE}$, $\mathrm{N}>300$. (D) Quantitation of WT dynein run length on MTs with (+) and without (-) the E-hook. Removal of the E-hook causes a two-fold decrease in run length. The difference is statistically significant (two-tailed KS-test, $\mathrm{P}<0.01$ ). (E) Quantitation of dynein run length on MTs without E-hooks. Note that CC1-E3289K causes a two-fold increase in run length and H6-E3378K causes a greater than three-fold increase in run length relative to WT. The increases are statiscally significant (two-tailed KS-test, $\mathrm{P}<0.01$ ). Data in (D) and (E) are plotted as characteristic run length $+/-\mathrm{SE}$. 
for its next binding site (Figure $2.13 \mathrm{C}$ ) an unbound MTBD is in the low-affinity conformation with its stalk in the $\beta+$ registry, H1 oriented perpendicular to the MT axis and intramolecular salt bridges at key MT-binding residues. Consistent with this, an NMR study found that an unconstrained, minimal MTBD in solution exists in the $\beta+$ registry and displays low affinity for MTs (47). Upon binding (Figure 2.13 D), transition to a high-affinity conformation involves a large displacement of $\mathrm{H} 1$, stabilized by new salt bridges with $\beta$-tubulin, and an opening of CC1 at the base of the stalk (Figure 2.13 E). The movements of $\mathrm{H} 1$ and $\mathrm{CC} 1$ likely constrain the registries that can be explored by the stalk, biasing the distribution towards the high-affinity $\alpha$ registry (Figure 2.13 E). Propagation of this signal to the head would elicit conformational changes that produce a movement of the linker domain, and a displacement of dynein towards the MT minus end.

Our analysis of dynamic salt bridges reveals that cytoplasmic dynein has been selected for sub-maximal processivity. While kinesin has diversified its functional repertoire through gene duplication and divergence (48), cytoplasmic dynein is expressed from a single locus and may have evolved sub-optimal processivity to increase the dynamic range of its regulation. High processivity could also be detrimental when multiple dyneins and kinesins must balance their actions on a single cargo (49). Consistent with this idea, intraflagellar dyneins, responsible for long, unidirectional transport within cilia $(50,51)$, contain neutral or basic residues at the equivalent of H6-E3378 (Figure 2.17), which would likely increase their processivity. 


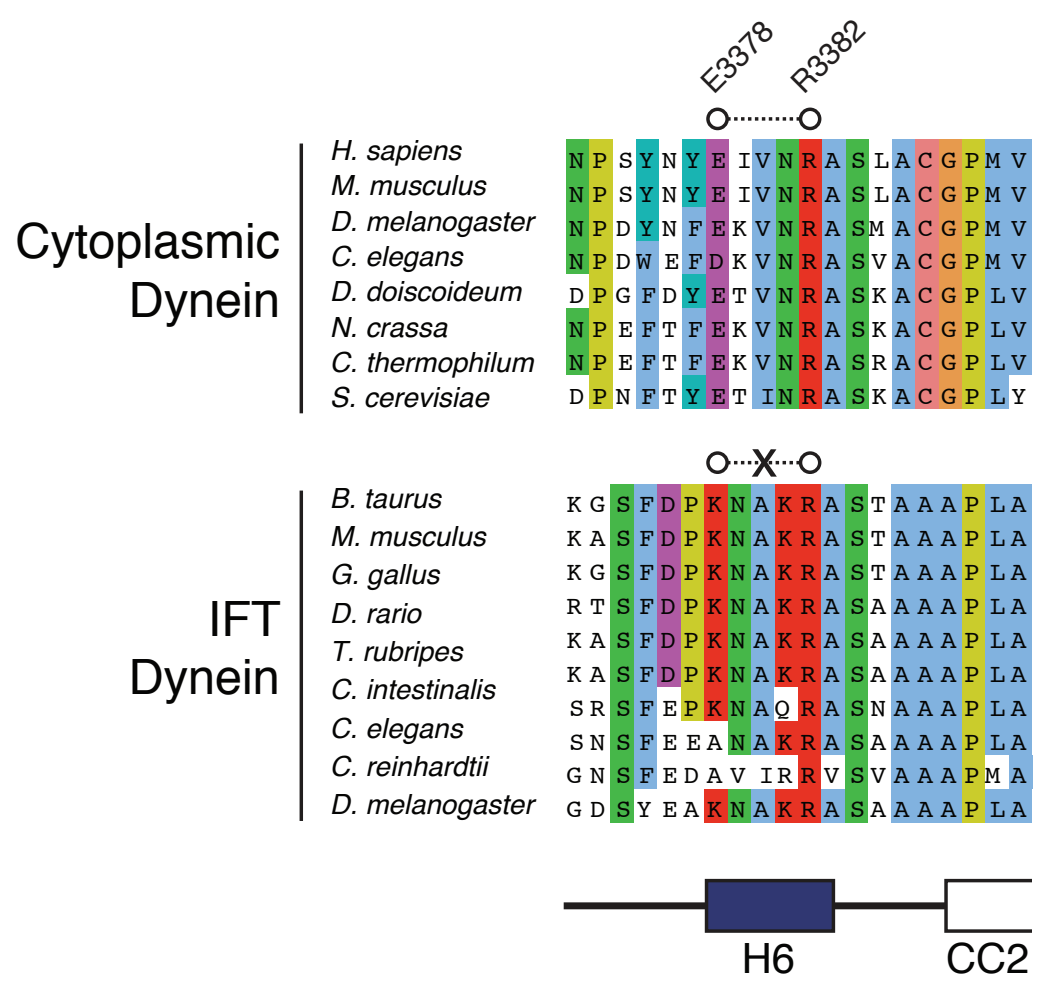

Figure 2.17. Intraflagellar transport dynein (cytoplasmic dynein 2) lacks a key intramolecular salt bridge that tunes the affinity of cytoplasmic dynein. Alignment of a section of the MTBD from diverse cytoplasmic and intraflagellar transport (IFT) dynein sequences calculated with MAFFT and visualized with Jalview according to the Clustal coloring scheme. An intramolecular salt bridge, whose disruption increases cytoplasmic dynein processivity (Figure 2.13), is comprised of Mus musculus (Mm) E3378 and R3382 and is indicated by a dashed line. Note that IFT dynein lacks an acidic residue at the position equivalent to $\mathrm{Mm}$ E3378, and this position is often basic. This would prevent formation of an intramolecular salt bridge in H6, and indicates that this configuration might enhance IFT dynein processivity. 


\section{Acknowledgements}

We thank A. Carter (LMB-MRC) for reagents and advice, C. Sindelar (Yale), V. Ramey (UC Berkeley), E. Egelman (U of Virginia), and R. Sinkovits (UCSD) for sharing processing scripts and helpful advice, M. Sotomayor (Harvard) and R. Gaudet (Harvard) for advice concerning MD, J. Hogle (Harvard), M. Strauss (Harvard) and M. Wolf (OIST) for help with film and the use of a film scanner, E. Nogales (UC-Berkeley), N. Francis (Harvard), and D. Pellman (Harvard) for critically reading the manuscript, as well as all the members of the Leschziner and Reck-Peterson Labs for advice and helpful discussions. EM data was collected at the Center for Nanoscale Systems (CNS), a member of the National Nanotechnology Infrastructure Network (NNIN), which is supported by the National Science Foundation under NSF award no. ECS-0335765. CNS is part of Harvard University. MD simulations were run on the Odyssey cluster supported by the FAS Science Division Research Computing Group, Harvard University. SRP is funded by the Rita Allen Foundation, the Harvard Armenise Foundation, and a NIH New Innovator award (1 DP2 OD004268-1). AEL was funded in part by a Research Fellowship from the Alfred P. Sloan Foundation. RHL was supported in part by CONACYT and Fundacion Mexico en Harvard. The cryo-EM map was deposited at the EM Data Bank (EMDB-5439) and pseudo-atomic models at the Protein Data Bank (PDB-3J1T and -3J1U). 


\section{References}

1. I. Gibbons, Dynein family of motor proteins: present status and future questions, Cell Motil Cytoskeleton 32, 136-144 (1995).

2. P. Höök, R. B. Vallee, The dynein family at a glance, J Cell Sci 119, 4369-4371 (2006).

3. R. D. Vale, The molecular motor toolbox for intracellular transport, Cell 112, 467-480 (2003).

4. R. B. Vallee et al., Emerging roles for myosin II and cytoplasmic dynein in migrating neurons and growth cones, Trends Cell Biol 19, 347-355 (2009).

5. R. B. Vallee et al., Dynein: An ancient motor protein involved in multiple modes of transport, J Neurobiol 58, 189-200 (2004).

6. S. A. Burgess et al., Dynein structure and power stroke, Nature 421, 715-718 (2003).

7. A. J. Roberts et al., AAA+ Ring and linker swing mechanism in the dynein motor, Cell 136, 485-495 (2009).

8. I. R. Gibbons et al., Multiple nucleotide-binding sites in the sequence of dynein beta heavy chain, Nature 352, 640-643 (1991).

9. T. Kon et al., Distinct functions of nucleotide-binding/hydrolysis sites in the four AAA modules of cytoplasmic dynein, Biochemistry 43, 11266-11274 (2004).

10. S. L. Reck-Peterson, R. D. Vale, Molecular dissection of the roles of nucleotide binding and 
hydrolysis in dynein's AAA domains in Saccharomyces cerevisiae, Proc Natl Acad Sci USA 101, $1491-1495$ (2004).

11. S. L. Reck-Peterson et al., Single-molecule analysis of dynein processivity and stepping behavior, Cell 126, 335-348 (2006).

12. T. Shima et al., Two modes of microtubule sliding driven by cytoplasmic dynein, Proc Natl Acad Sci USA 103, 17736-17740 (2006).

13. A. P. Carter et al., Crystal structure of the dynein motor domain, Science 331, 1159-1165 (2011).

14. T. Kon et al., The $2.8 \AA$ crystal structure of the dynein motor domain, Nature 484, 345-350 (2012).

15. M. P. Koonce, Identification of a Microtubule-binding Domain in a Cytoplasmic Dynein Heavy Chain, Journal of Biological Chemistry 272, 19714-19718 (1997).

16. M. A. Gee et al., An extended microtubule-binding structure within the dynein motor domain, Nature 390, 636-639 (1997).

17. A. P. Carter et al., Structure and functional role of dynein's microtubule-binding domain, Science 322, 1691-1695 (2008).

18. I. R. Gibbons et al., The affinity of the dynein microtubule-binding domain is modulated by the conformation of its coiled-coil stalk, J Biol Chem 280, 23960-23965 (2005).

19. T. Kon et al., Helix sliding in the stalk coiled coil of dynein couples ATPase and microtubule 
binding, Nat Struct Mol Biol 16, 325-333 (2009).

20. N. Mizuno et al., Dynein and kinesin share an overlapping microtubule-binding site, $E M B O$ $J$ 23, 2459-2467 (2004).

21. S. J. Ludtke et al., EMAN: semiautomated software for high-resolution single-particle reconstructions, J Struct Biol 128, 82-97 (1999).

22. V. H. Ramey et al., Ab initio reconstruction of helical samples with heterogeneity, disorder and coexisting symmetries, J Struct Biol 167, 97-105 (2009).

23. G. M. Alushin et al., The Ndc 80 kinetochore complex forms oligomeric arrays along microtubules, Nature 467, 805-810 (2010).

24. C. V. Sindelar, K. H. Downing, The beginning of kinesin's force-generating cycle visualized at 9-A resolution, $J$ Cell Biol 177, 377-385 (2007).

25. C. V. Sindelar, A seesaw model for intermolecular gating in the kinesin motor protein, Biophys Rev 3, 85-100 (2011).

26. N. Grigorieff, FREALIGN: high-resolution refinement of single-particle structures, $J$ Struct Biol 157, 117-125 (2007).

27. J. Löwe et al., Refined structure of alpha beta-tubulin at 3.5 A resolution, $J$ Mol Biol 313, 1045-1057 (2001).

28. J. A. Mindell, N. Grigorieff, Accurate determination of local defocus and specimen tilt in electron microscopy, J Struct Biol 142, 334-347 (2003). 
29. N. Grigorieff, S. C. Harrison, Near-atomic resolution reconstructions of icosahedral viruses from electron cryo-microscopy, Curr Opin Struct Biol 21, 265-273 (2011).

30. X. Zhang et al., Near-atomic resolution using electron cryomicroscopy and single-particle reconstruction, Proc Natl Acad Sci USA 105, 1867-1872 (2008).

31. E. H. Egelman, A robust algorithm for the reconstruction of helical filaments using singleparticle methods, Ultramicroscopy 85, 225-234 (2000).

32. C. V. Sindelar, K. H. Downing, An atomic-level mechanism for activation of the kinesin molecular motors, Proc Natl Acad Sci USA 107, 4111 (2010).

33. E. F. Pettersen et al., UCSF Chimera--a visualization system for exploratory research and analysis, J Comput Chem 25, 1605-1612 (2004).

34. D. B. Wells, A. Aksimentiev, Mechanical properties of a complete microtubule revealed through molecular dynamics simulation, Biophysical Journal 99, 629-637 (2010).

35. L. G. Trabuco et al., Flexible fitting of atomic structures into electron microscopy maps using molecular dynamics, Structure 16, 673-683 (2008).

36. L. G. Trabuco et al., Molecular dynamics flexible fitting: a practical guide to combine cryoelectron microscopy and X-ray crystallography, Methods 49, 174-180 (2009).

37. J. Schlitter et al, Targeted molecular dynamics: a new approach for searching pathways of conformational transitions, J Mol Graph 12, 84-89 (1994).

38. J. C. Phillips et al., Scalable molecular dynamics with NAMD, J Comput Chem 26, 1781- 
1802 (2005).

39. A. D. Mackerell et al., Empirical force fields for biological macromolecules: overview and issues, J Comput Chem 25, 1584-1604 (2004).

40. W. L. Jorgensen et al., Comparison of simple potential functions for simulating liquid water, J. Chem. Phys. 79, 926 (1983).

41. W. Humphrey et al., VMD: visual molecular dynamics, J Mol Graph 14, 33-8, 27-8 (1996).

42. W. Qiu et al., Dynein achieves processive motion using both stochastic and coordinated stepping, Nat Struct Mol Biol 19, 193-200 (2012).

43. R. B. Case, et al., The directional preference of kinesin motors is specified by an element outside of the motor catalytic domain, Cell 90, 959-966 (1997).

44. S. Uchimura et al., Key residues on microtubule responsible for activation of kinesin ATPase, EMBO J 29, 1167 (2010).

45. M. P. Koonce, I. Tikhonenko, Functional elements within the dynein microtubule-binding domain, Mol Biol Cell 11, 523-529 (2000).

46. Z. Wang, M. P. Sheetz, The C-terminus of tubulin increases cytoplasmic dynein and kinesin processivity, Biophysical Journal 78, 1955-1964 (2000).

47. L. Mcnaughton et al., A Low Affinity Ground State Conformation for the Dynein Microtubule Binding Domain, Journal of Biological Chemistry 285, 15994-16002 (2010). 
48. E. M. Dagenbach, S. A. Endow, A new kinesin tree, J Cell Sci 117, 3-7 (2004).

49. M. A. Welte, Bidirectional transport along microtubules, Curr Biol 14, R525-37 (2004).

50. C. Iomini et al., Protein particles in Chlamydomonas flagella undergo a transport cycle consisting of four phases, J Cell Biol 153, 13-24 (2001).

51. J. A. Laib et al., The reciprocal coordination and mechanics of molecular motors in living cells, Proc Natl Acad Sci USA 106, 3190-3195 (2008). 


\section{Chapter 3}

Consequences of enhanced dynein processivity in vivo

William Bret Redwine, Brian Goodman, Samara Reck-Peterson 


\section{Contributions}

William Redwine and Brian Goodman initiated the project. William Redwine and Brian Goodman collected data. Brian Goodman wrote custom scripts for processing. William Redwine and Brian Goodman analyzed data. Samara Reck-Peterson provided mentorship. 


\begin{abstract}
In Chapter 2 we described a sub-nanometer cryo-EM reconstruction of the high affinity state of dynein's MTBD bound to MTs. We also presented a pseudoatomic model that was the result of MDFF with a previously reported low affinity crystal structure of the MTBD. Surprisingly, we found evidence that dynein is an inherently restrained motor, with a basal processivity much lower than what is accomplished by individual amino acid changes. Here we describe our efforts to determine what effect high processivity mutations have on dynein function in vivo in $S$. cerevisiae. S. cerevisiae utilizes a dynein-based pathway for mitotic spindle positioning and elongation. We find that dynein-driven nuclear movements are generally impaired in strains expressing highly processive mutants, and we outline a strategy to gain further insight into this impairment.
\end{abstract}




\section{Introduction}

Budding yeast provides an important biological system to explore the function and regulation of cytoplasmic dynein (1). Yeast express a single cytoplasmic dynein from the DYN1 locus $(2,3)$. In a departure from higher eukaryotes, dynein is not known to transport cargo in yeast; instead myosin moves along actin filaments to fulfill this role $(4,5)$. Dynein's primary role in yeast is to position the mitotic spindle at the point of cell division, the bud neck. Loss of dynein function results in an increase in the number of cells that fail to segregate nuclei properly, increasing the number of binucleate mother cells and anucleate daughter cells $(3,6)$. In spite of its lack of transport activity, purified dynein, either in its full length form with associated factors, or as a truncated "minidimer", is capable of walking along microtubules in vitro (7). Since dynein in yeast is capable of motility yet devoid of cargo, if it were free to associate with and walk along microtubules it would do so without accomplishing anything of cellular importance. To prevent this, yeast have developed a number of mechanisms to ensure dynein is localized and activated in a tightly controlled manner $(8,9)$.

Yeast undergo a closed mitosis, meaning that the nuclear envelope remains intact throughout the cell cycle; spindle elongation, therefore, is largely responsible for segregating the correct number of chromosomes to each cell. Under these circumstances, it is important that the mitotic spindle be correctly positioned at the bud neck. Central to this process are large structures known as spindle pole bodies (SPBs) that are equivalent to the centrosomes found in higher eukaryotes (10). SPBs lie embedded in the nuclear envelope and contain both nucleoplasmic as well as cytoplasmic faces (11). Microtubules emanate from each face of the $\mathrm{SPB}$, and constitute what are referred to as spindle and astral microtubules that lie in the nucleus 
and cytoplasm, respectively. Spindle microtubules attach to chromosomes via the kinetochore, where the force generated by microtubule depolymerization is harnessed to separate sister chromatids (12). Astral, or cytoplasmic microtubules, grow and shrink owing to their inherent dynamic instability (13), searching out the cell cortex in the process (14). Dynamic astral microtubules have at their tips an inactive form of cytoplasmic dynein, which, through interactions between its tail and the membrane protein Num1, anchors the microtubule to the cell cortex (15-17). There, in a manner akin to in vitro MT sliding assays, dynein's restricted motility is translated into microtubule pulling, and the movement of an attached SPB towards the bud neck (18) (Figure 3.1 A). Cortical pulling by dynein is also utilized by higher eukaryotes to position their SPB equivalent, the centrosome, suggesting some of the mechanisms in yeast might be conserved (19).

In yeast, accurate partitioning of a single nucleus into each of the mother and daughter cells is accomplished in roughly three steps: nuclear migration to the bud site, persistence at the bud neck, and nuclear segregation into the two cells (9). Early in G1, cells with nascent buds use a pathway centered on the Bud6 protein to ensure that the nucleus migrates to the bud site (20). Next, overlapping pathways (Kar9 and dynein, Figure 3.1) ensure that the replicating nucleus persists at the bud neck (9). Lastly, as growing polar microtubules cause the spindle to elongate, pulling forces from both cell cortices segregate the now divided nucleus. Dynein has been implicated in each of these processes. While a newly described role in nuclear migration within the context of Bud6 is still unclear (21), it is well established that dynein accomplishes persistence and segregation tasks through tightly controlled localization (22). During metaphase, dynein is recruited to the daughter cortex, where it selectively pulls on microtubules emanating 
from the daughter proximal SPB. This drives the movement of the mitotic spindle to the bud neck, a process that is redundantly accomplished through connections that link astral microtubules to the actin network via a Kar9-myosin interaction (Figure 3.1). Later, during anaphase, dynein appears at foci along the mother cell cortex, and begins to evenly pull from both sides, aiding in spindle elongation and nuclear segregation (Figure 3.1 C) (22).

Severe defects in the dynein pathway (i.e. the deletion of dynein itself or components of the dynactin complex) manifest themselves as an increase in the number of cells that fail to partition one nucleus into the daughter cell. This causes an increase in the number of binucleate mother cells $(2,3)$. As a preliminary test, we determined whether enhanced processivity dynein mutants (Chapter 2) caused a binucleate phenotype. We found no increase in the number of binucleate cells as compared to WT cells (data not shown). In this chapter we report our use of hydroxyurea to arrest cells in pre-anaphase, which allowed us to monitor spindle dynamics in vivo. This assay has proven effective for characterizing mutants that exhibit no binucleate phenotype $(23,24)$. We applied single-particle tracking programs to the movement of SPBs, and were able to quantitatively determine SPB velocity, bud neck crossings, and localization to different cellular compartments. We found that highly processive dynein mutants exhibit defective pre-anaphase spindle dynamics, and we also outline a strategy to elucidate the mechanism underlying these defects. 
A

B
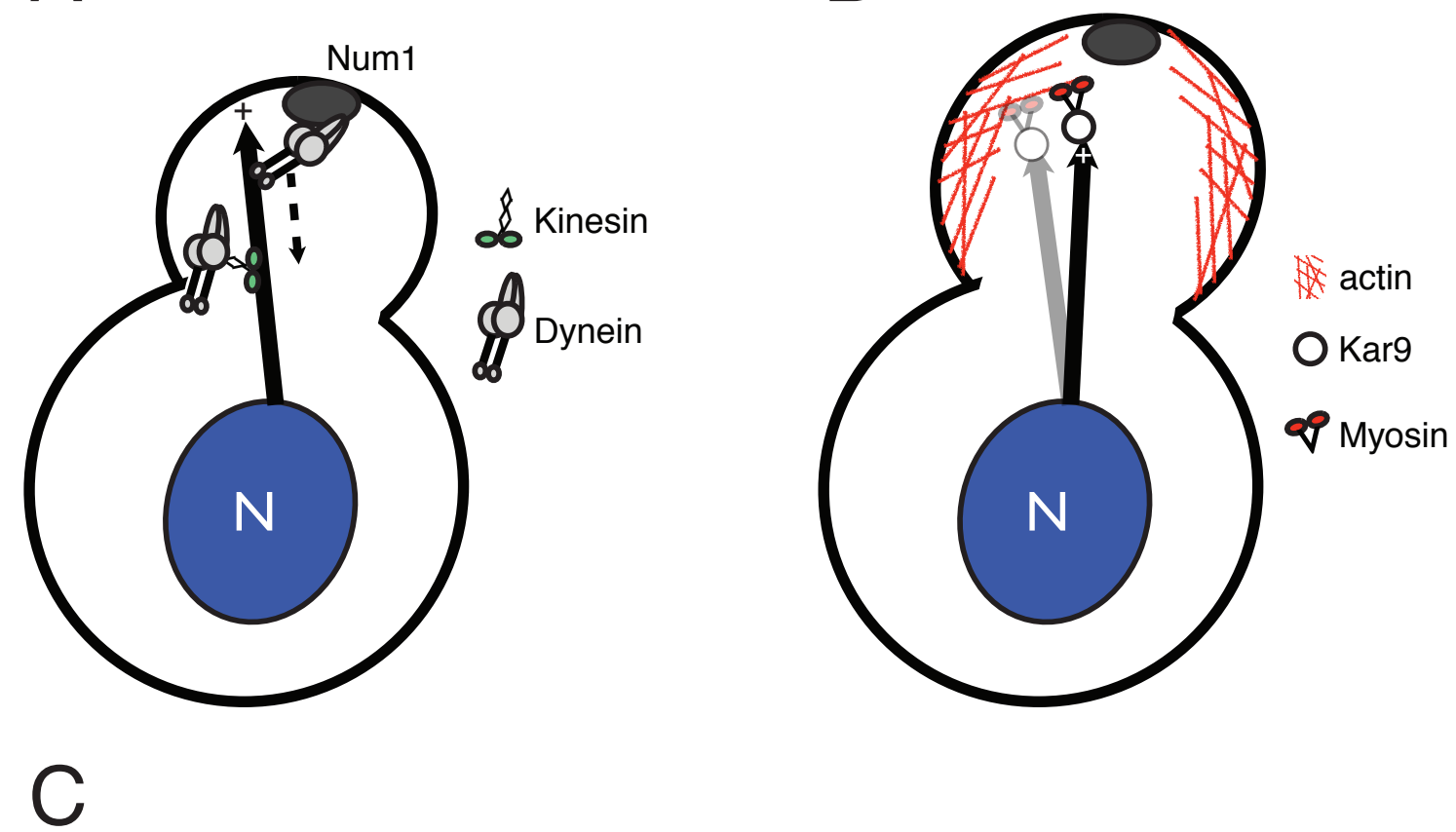

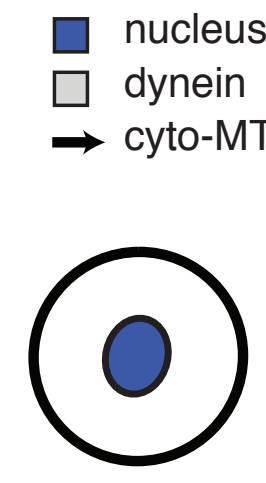

1

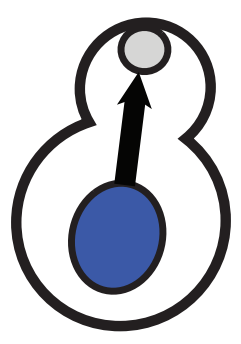

2

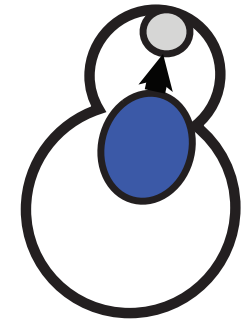

3

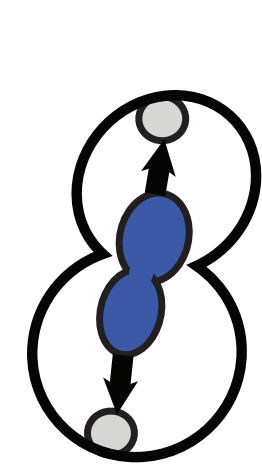

4

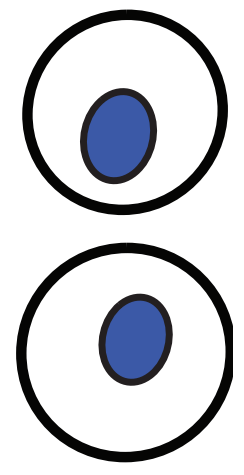

5

Figure 3.1 Two pathways contribute to maintenance of the mitotic spindle at the bud neck. (A) The dynein pathway relies upon localization of dynein to the daughter cell cortex. In part, dynein is transported by kinesin (Kip2) towards the plus end of astral microtubules (arrow, indicates direction of nuclear movement). There it is unloaded and interacts with patches of the Num1 membrane protein. (B) Redundant with the dynein pathway, the Kar9 pathway utilizes a connection between microtubules and a polarized actin network in the daughter cell. Kar9, which interacts with a plus-end binding protein, also interacts with a myosin (Myo2). This connection allows myosin to pull astral MTs and the attached nucleus towards the daughter cell cortex. (C) Dynein also aides in nuclear segregation. Its localization is asymmetric during metaphase (A) and (2), and then symmetric during anaphase (4). 


\section{Materials and methods}

\section{Strain construction}

The starting strains for this study, RPY1245 and RPY1246, which express SPC110-GFP (spindle pole body component) and HXT1-tdTomato (plasma membrane marker) from their endogenous loci, were the kind gifts of Jeff Moore (University of Colorado, Denver). Mutations in the MTBD of dynein were accomplish by sequential URA3 replacement of the MTBD, transformation with mutant MTBD sequence, and 5FOA selection. First, the MTBD encoding portion of the DYN1 locus (nucleotides 5648-5996) was replaced with URA3 (mtbdA::URA3). Insertion of URA3 was verified by genomic PCR. A larger portion of the DYN1 locus (nucleotides 5496-6125) was cloned into pCRII-TOPO (Invitrogen) and sequence verified. This plasmid was then subjected to site-directed mutagenesis, and mutations were sequence verified. The strain containing $m t b d \Delta::$ URA3 was then transformed with linearized plasmids containing each desired mutation. After two rounds of selection on plates containing 5FOA colonies were screen by genomic PCR for loss of the URA3 cassette. PCR products were then sequenced to verify insertion of the desired mutation. These strains were then transformed with kar9 $9::$ HygroB to generate a second set of strains lacking KAR9 (see Table 3.1 for strain details).

\section{Growth and imaging conditions}

For spindle pole oscillation assays overnight cultures were diluted to an $\mathrm{OD}_{600}$ of 0.1 in a total volume of $10 \mathrm{~mL}$ YPD. The cultures were then incubated with rotation at $30{ }^{\circ} \mathrm{C}$ until they 
reached an $\mathrm{OD}_{600}$ of $0.2 .5 \mathrm{~mL}$ of this culture was transferred to a fresh tube, and $550 \mu \mathrm{L}$ of $2 \mathrm{M}$ hydroxyurea (HU) was added (200 mM final concentration). The culture was incubated for an additional 1.5 hours with rotation at $30^{\circ} \mathrm{C}$. The cells were collected by centrifugation at 4000 rpm for 5 minutes, the media was discarded, and the cells were transferred to an Eppendorf tube with $1 \mathrm{~mL}$ of synthetic complete yeast media (SC) containing $200 \mathrm{mM} \mathrm{HU}$. The cells were quickly centrifuged in a microcentrifuge, and then resuspended to a total volume of $500 \mu \mathrm{L}$ with $\mathrm{SC}+\mathrm{HU}$. The cells were loaded into Y04C microfluidic yeast plate (CellASIC) and introduced into the viewing chamber with the ONIX controller (CellASIC). The plate was mounted and imaged with a Nikon Ti inverted microscope equipped with a Yokagawa CSU-10 spinning disk confocal system, $100 \mathrm{~mW}$ Melles-Griot Argon-Krypton laser, Hamamatsu ORCA-AG CCD, and MetMorph software (Nikon Imaging Center, Harvard Medical School). Images were collected for each fluor (excitation $488 \mathrm{~nm}$ and $568 \mathrm{~nm}$ for GFP and tdTomato, respectively) as 100-200 ms exposures spanning ten $300 \mathrm{~nm} Z$-sections ( $3 \mu \mathrm{m}$ total $\mathrm{Z}$ stack), with 20 seconds between $\mathrm{Z}$ series, and a total duration of 20 minutes.

\section{Image processing and particle tracking}

Maximum projections of the Z-series at each time point of the spindle channel were performed for analysis. Each spindle pole was detected in the z-projection of each time point using a wavelet detection algorithm (Francois Aguet, unpublished Matlab (Mathworks) scripts) and the two spindles were tracked throughout the course of the movie using a nearest neighbor tracking method (Brian Goodman, unpublished Matlab (Mathworks) scripts). The location of the bud neck and the mother-daughter orientation were determined using either the DIC image of the 
cell or the membrane marker. Locations of tracked spindle poles relative to the defined bud neck were analyzed for velocity, orthogonal distance to the bud neck, and relative time spent in the mother cell, daughter cell, or spanning the bud neck.

Note: Because in some sections gene loci and proteins with the same name are discussed together, we use the yeast community naming convention to avoid confusion. As an example consider yeast dynein, which has the four letter designation dyn1: WT genomic locus $=$ DYN1, locus deleted $=d y n 1 \Delta$, protein $=$ Dyn 1. 
Table 3-1. Yeast strains used in this study. All yeast strains are based on the W303 background.

\begin{tabular}{|c|c|c|c|}
\hline Strain ID & Genotype & Source & Figure \\
\hline yJM165 & 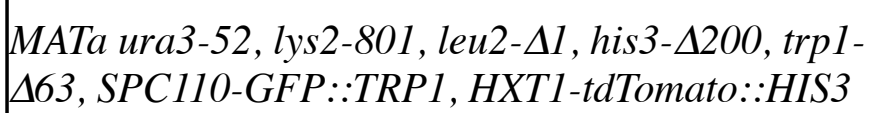 & Jeff Moore & $\begin{array}{l}3.2,3.3 \\
3.5-7\end{array}$ \\
\hline yJM208 & 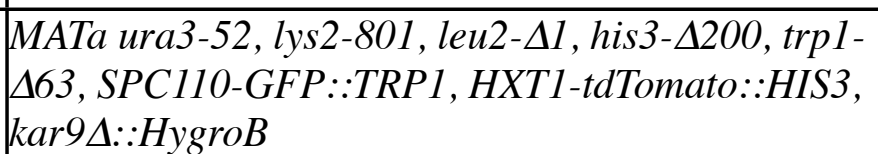 & Jeff Moore & $\begin{array}{l}3.2,3.3 \\
3.5-7\end{array}$ \\
\hline RPY1248 & 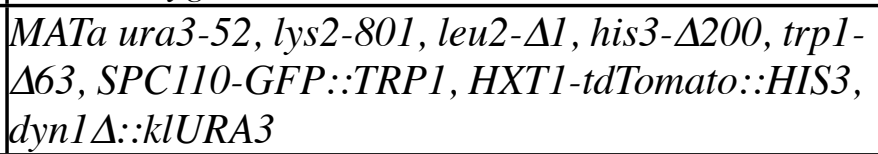 & this study & $\begin{array}{l}3.2,3.3 \\
3.5-7\end{array}$ \\
\hline RPY1251 & 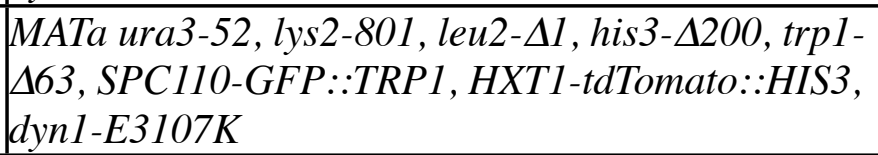 & this study & $\begin{array}{l}3.2,3.3 \\
3.5-7\end{array}$ \\
\hline RPY 1253 & 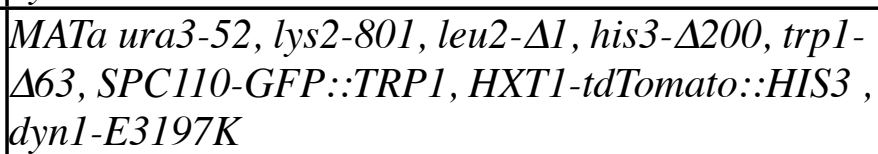 & this study & $\begin{array}{l}3.2,3.3 \\
3.5-7\end{array}$ \\
\hline RPY1254 & $\begin{array}{l}\text { MATa ura3-52, lys } 2-801, \text { leu2- } \Delta 1, \text { his3- } \triangle 200, \text { trpl- } \\
\Delta 63, \text { SPC110-GFP::TRP1, HXT1-tdTomato::HIS3, } \\
\text { dyn1-E3107K/E3197K }\end{array}$ & this study & $\begin{array}{l}3.2,3.3 \\
3.5-7\end{array}$ \\
\hline RPY 1268 & 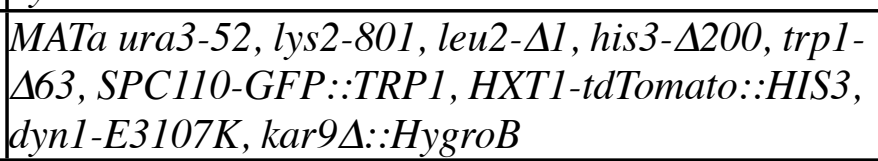 & this study & $\begin{array}{l}3.2,3.3 \\
3.5-7\end{array}$ \\
\hline RPY1270 & 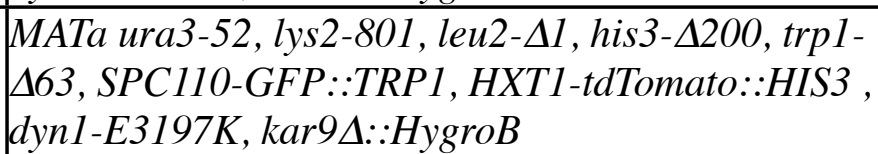 & this study & $\begin{array}{l}3.2,3.3 \\
3.5-7\end{array}$ \\
\hline RPY1271 & 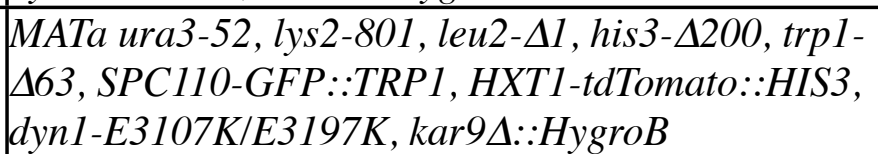 & this study & $\begin{array}{l}3.2,3.3 \\
3.5-7\end{array}$ \\
\hline RPY1331 & $\begin{array}{l}\text { Mata lys2-801 leu2-D1 his3-D200 trp1-D63 } \\
\text { DYN1-3GFP::TRP1, ura3-52::CFP-TUB1::URA3, } \\
\text { SPC110-tdTomato::SpHIS5 }\end{array}$ & this study & 3.4 \\
\hline
\end{tabular}




\section{Results}

In order to better understand why yeast dynein has been selected for sub-maximal motility, we introduced high processivity mutations into the MTBD (E3107K, E3197K, and the combination E3107K + E3197K, see Chapter 2 for details), and determined their effect on dynein-driven nuclear oscillations in yeast. The mutations were introduced into a parent strain expressing from their endogenous loci GFP-Spc110, a spindle pole body component (SPB) that lies at the base of the gamma-TURC microtubule nucleating complex $(10,25)$; and Hxt1tdTomato, a glucose transporter that serves as a marker for the plasma membrane (26). We arrested cells in pre-anaphase with hydroxyurea (HU) and imaged live cells in a microfluidics chamber designed to reduce cell drift during extended data collection. First, we overlaid consecutive images of SPBs (captured $20 \mathrm{sec}$ apart) on an image of the plasma membrane (Figure 3.2). The SPBs appeared as bright, distinct punctae with no apparent background fluorescence, which demonstrated that SPBs are suitable for automated particle tracking. We collected extended movies of immobilized HU-arrested cells, and used automated particle tracking algorithms to follow the movement of SPBs in vivo (Figure 3.3). We observed large displacements of the mitotic spindle, which constitutes the area between SPBs, across the bud neck. The SPB pairs moved in unison, with little or no spindle elongation occurring. The bidirectional movement of the spindle suggested that in HU-arrested cells dynein is localized to, and alternately pulls from, each cell cortex. This is supported by images of HU-arrested cells simultaneously expressing SPC110-tdTomato, CFP-tubulin and GFP-dynein. In these cells we observed numerous cortical dynein patches at the point of contact with astral microtubules in mother and daughter cells (Figure 3.4). This suggested that dynein was symmetrically localized 

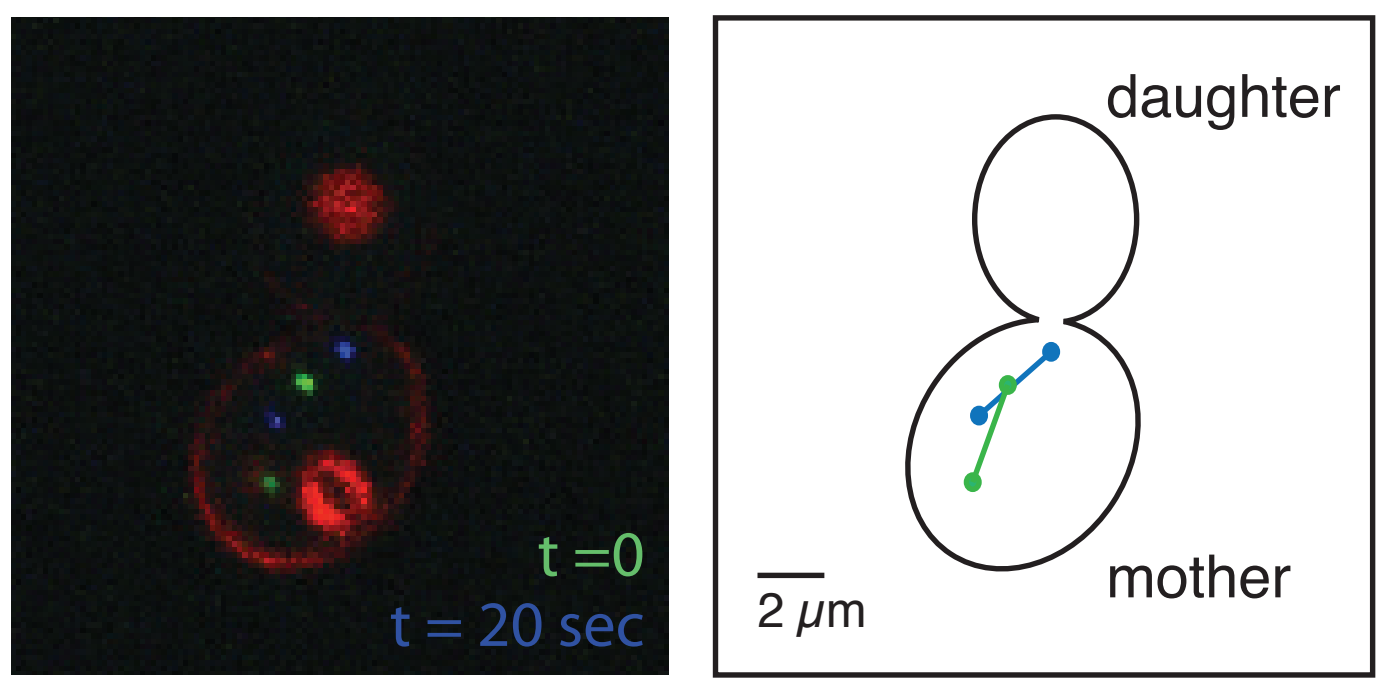

Figure 3.2 Imaging dynein-driven spindle movements in vivo. (Left) Overlay of two consecutive time points. The plasma membrane is labeled with Hxt1tdTomato (red). The spindle pole bodies (SPBs) are labelled with GFP-Spc110, and are colored according to the time point (green $=$ start of imaging, blue $=20$ seconds). (Right) Interpretation of the image at left. The mitotic spindle spans the region between SPBs, and according to its time point. The mother cell is identified by higher levels of Hxt1-tdTomato expression. Note the nearly $2 \mu \mathrm{m}$ movement of the spindle towards the daughter cell. The cell shown is expresses WT dynein. 

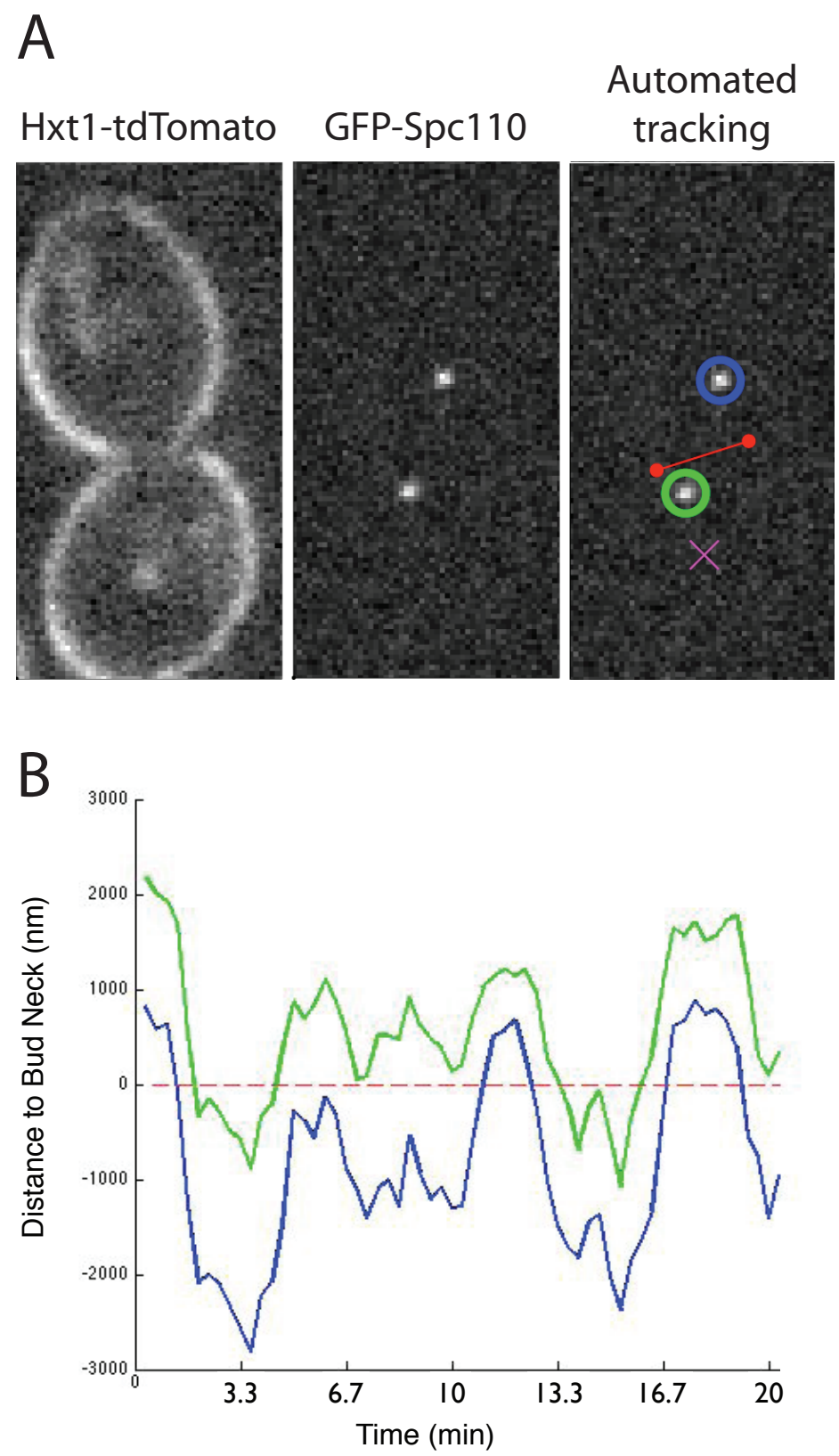

Figure 3.3. Automated tracking of spindle pole bodies in vivo. (A) Schematic of automated tracking routine. Hxt1-tdTomato images (left panel) are used to determine the position of the bud neck (red line, right panel). GFP-Spc110 punctae, visualized as maximum projections of an entire Z-stack at each time point, are tracked individually (green and blue circles, right panel). (B) Representative trace of the movement of individual spindle pole bodies (green and blue lines) relative to the bud neck (red dashed line). 

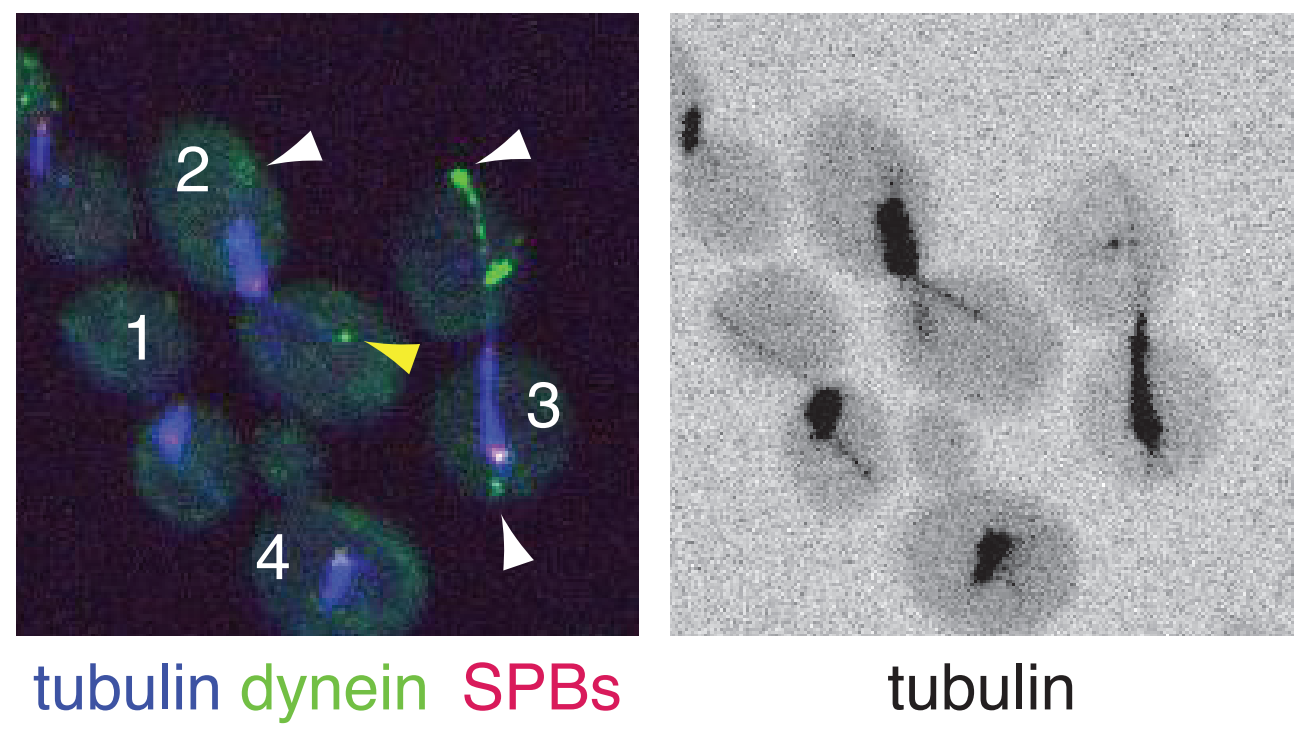

\section{tubulin}

Figure 3.4. Symmetrically localized dynein in HU-arrested cells. (left)

Maximum projection of a Z-stack of a strain expressing GFP-dynein (green), CFP-tubulin (blue), and Spc110-tdTomato (red) and arrested in pre-anaphase. White arrows in cell 2 and 3 point to cortical dynein patches. Yellow arrow points to a MT plus end with co-localized dynein. (right) Grayscale image of the CFP channel from the image at left. Contrast has been enhanced to make astral MTs more visible. Note the MTs associating with the dynein patches indicated at left. 
as reported for cells arrested in pre-anaphase (22). Additionally, to distinguish dynein-driven movements from the combination of dynein- and myosin-driven (via the Kar9 pathway, Figure 3.1) movements, we collected data for each MTBD mutant in a WT background, and in strains with the KAR9 locus deleted ( $\operatorname{kar} 9 \Delta)$. For each background we determined the effect of MTBD mutations on various indicators of SPB movement, including velocity, bud neck crossings, and time spent in each the mother cell, daughter cell, and at the bud neck.

We first determined the effect of MTBD mutations on the in vivo velocity of the mitotic spindle. In KAR9 cells we were able to include a negative control, which consisted of deletion of the entire DYN1 locus $(d y n 1 \Delta)$. We were not able to include this control in kar9 $9 \Delta$ cells, as the combined deletion of KAR9 and DYN1 is synthetically lethal (27). In KAR9 cells, each of the three MTBD high processivity mutants reduced the velocity of mitotic spindles to levels similar to that observed in $d y n 1 \Delta$ cells (Figure 3.5). This represented an approximately $30 \%$ reduction compared to WT cells. A similar reduction relative to WT cells was observed in strains lacking Kar9 (Figure 3.5 B).

Next we determined the number of times the mitotic spindle oscillated across the bud neck. Single mutations (E3107K, E3197K) within the MTBD caused a reduction in the number of times the spindle crossed the bud neck (Figure 3.6). In KAR9 cells, the reduction was modest for the $\mathrm{E} 3107 \mathrm{~K}$ mutation, while the E3197K mutation reduced the number of bud neck crossings nearly to the level observed in $d y n 1 \Delta$ cells (Figure 3.6 A). Interestingly, the double mutant E3107K/E3197K exhibited less severe bud neck crossing defects, most notably in kar9A cells where it exhibited a crossing frequency similar to WT (Figure 3.6 B). 

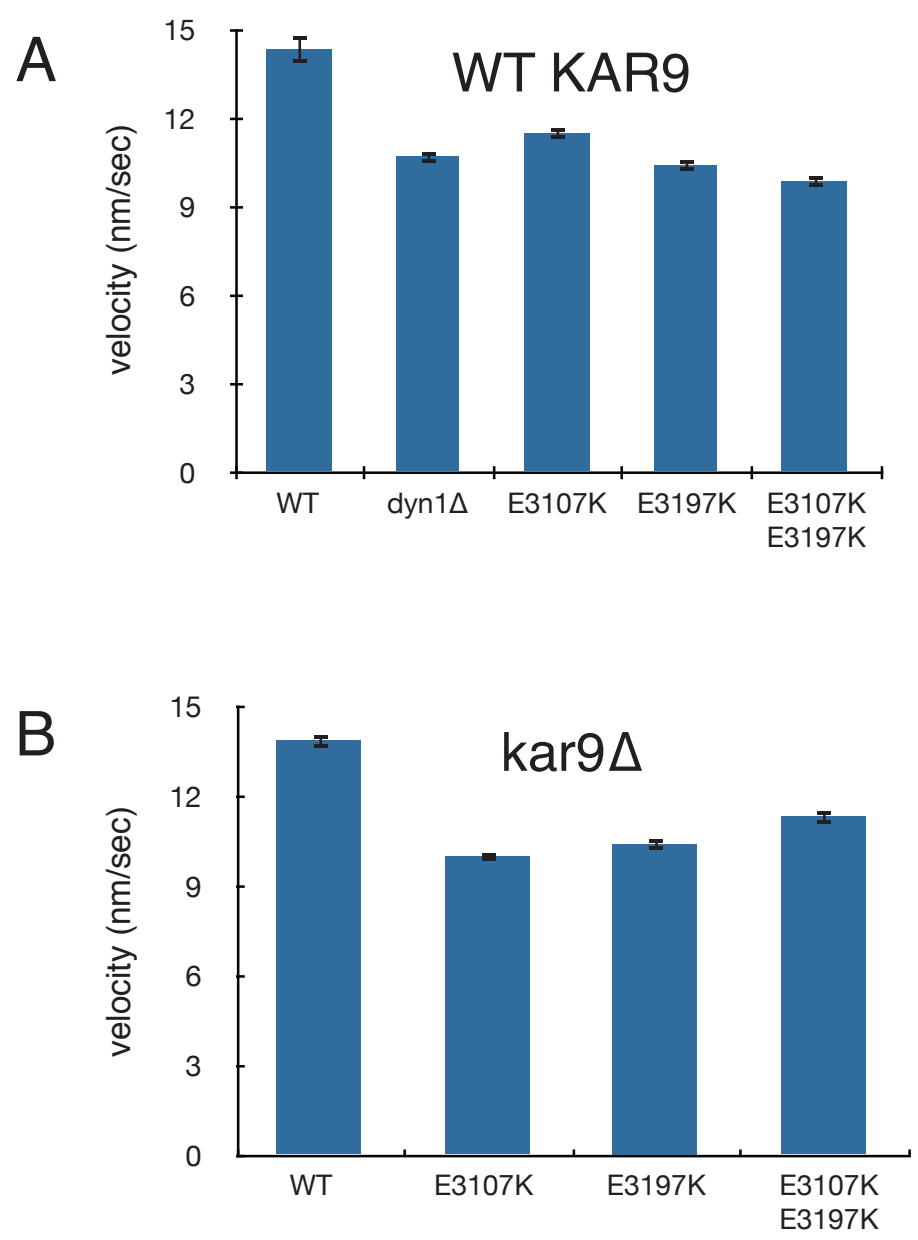

Figure 3.5. Highly processive dynein mutations reduce the velocity of mitotic spindle movement in vivo. (A) Velocity of Spc110-GFP tagged SPBs was determined for strains expressing WT and highly processive mutant dyneins (E3107K, E3197K, E3107K/E3197K). Strains lacking dynein (dyn1 $)$ were included as a negative control. (B) same as (A) with the addition of kar9A. Dynein deletion is not possible in this strain background, as the dyn $1 \Delta$ kar9 $9 \Delta$ combination is synthetically lethal. Error bars are standard error. 

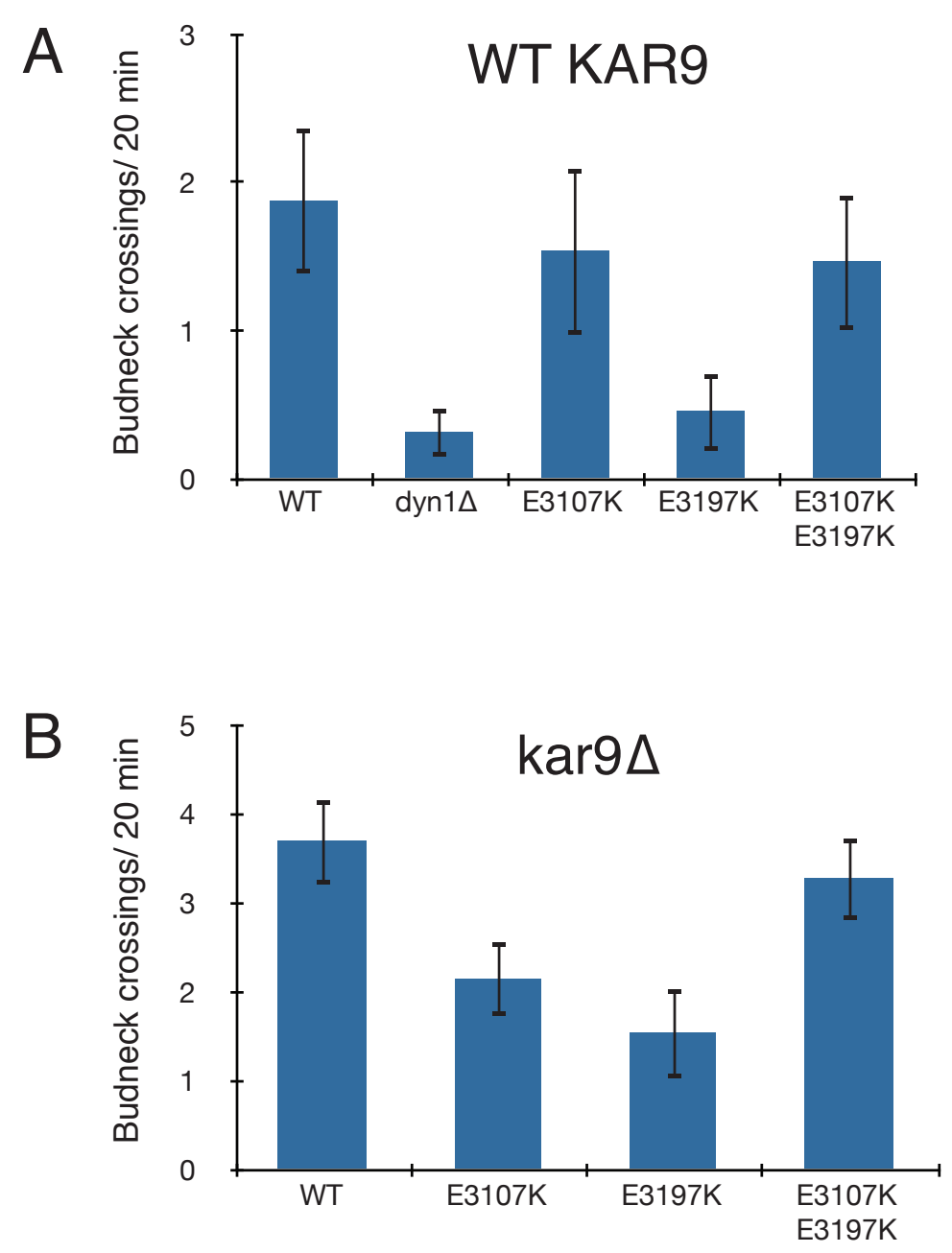

Figure 3.6. The effect of highly processive dynein mutations of mitotic spindle bud neck crossings in vivo. (A) The number of times Spc110-GFP tagged SPBs crossed the bud neck during 20 minute movies was determined for strains expressing WT and highly processive mutant dyneins (E3107K, E3197K, E3107K/E3197K). Strains lacking dynein (dyn1A) were included as a negative control. (B) same as (A) with the addition of kar9 $9 \Delta$. Dynein deletion is not possible in this strain background, as the dyn $1 \Delta$ kar $9 \Delta$ combination is synthetically lethal. Error bars are standard error. 
Lastly, we determined the proportion of time the mitotic spindle spent in each of three compartments: the mother cell, daughter cell, and the bud neck (Figure 3.7). In KAR9 cells, both the single mutations to the MTBD as well as their combination caused a decrease in the time the spindle spent spanning the bud neck. This occurred with a concomitant increase in the time spend in the mother cell. The same was largely true in kar9 $\Delta$ cells, again with the exception of the double mutant, which exhibit localization characteristics very similar to WT cells. 


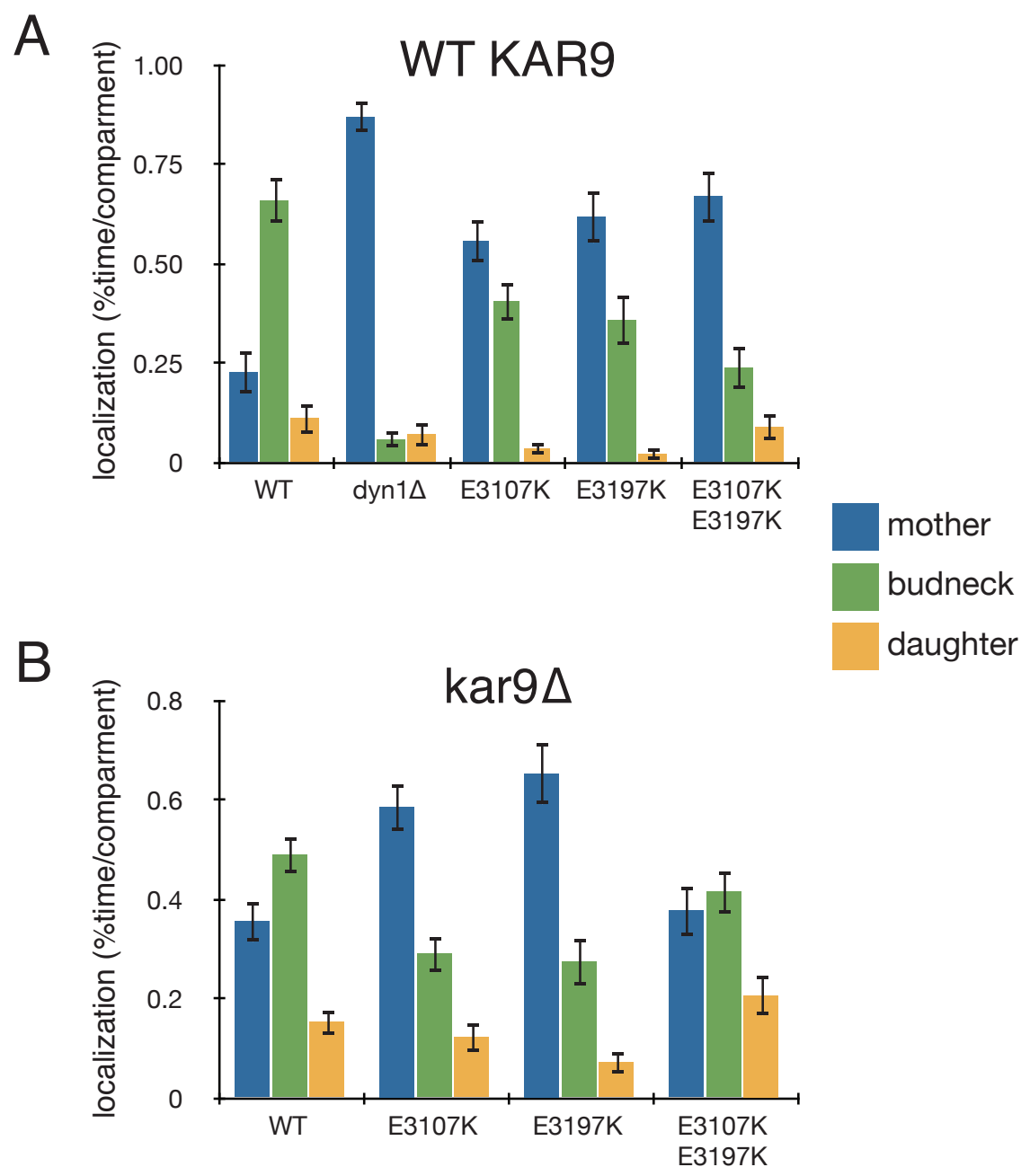

Figure 3.7. The effect of highly processive dynein mutations of mitotic spindle localization in vivo. (A) The percentage of time Spc110-GFP tagged SPBs spent in the mother (blue), daughter (yellow), and bud neck (green) was determined for strains expressing WT and highly processive mutant dyneins (E3107K, E3197K,

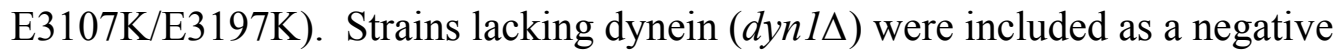
control. (B) same as (A) with the addition of kar $9 \Delta$. Dynein deletion is not possible in this strain background, as the dyn $1 \Delta$ kar $9 \Delta$ combination is synthetically lethal. Error bars are standard error. 


\section{Discussion and Future Directions}

Here we have described our efforts to determine what effects high processivity mutations within dynein's MTBD have on nuclear position in yeast. We found that the dynamics of spindle oscillations in HU-treated cells, which arrests cells in pre-anaphase, are perturbed in MTBD mutants. Mirroring our results with in vitro motility assays (Chapter 2), MTBD mutants exhibited lower velocities in vivo, which in turn likely resulted in the observed decrease in bud neck crossings. Overall, MTBD mutants also disrupted the localization of the spindle to the bud neck. A notable exception to the trends above was the double mutant, which combined mutations at H1 (E3107K) and H6 (E3197K). This mutant exhibited bud neck crossing similar to WT strains regardless of whether Kar9 was present. It also had a less severe spindle localization defect than strains harboring either single mutation; however, this defect dependent upon the absence of Kar9.

Budding yeast expend a lot of energy across at least three pathways (Bud6, Kar9, and dynein) to maintain the mitotic spindle at the cell division site, the bud neck. Why then would MTBD mutants spend less time with their spindles localized to the bud neck? One possibility is related to the nature of $\mathrm{HU}$ arrest. In HU-arrested cells spindle elongation is inhibited, but the dynein pathway has proceeded to a point that dynein is symmetrically localized to the mother and daughter cortices (Figure 3.4). There, via interactions with astral microtubules, it is fully competent to exert pulling forces in either direction. The large oscillations observed, most notably when the Kar9 pathway is absent, can likely be attributed to the loss of either a dyneincortex or dynein-microtubule interaction. If the loss of contact is due to a dissociation of dynein from the microtubule, we would predict that this would occur with less frequency in high 
processivity dynein mutants. Analysis of triple-tagged strains (CFP-tubulin, GFP-dynein, SPC110-tdTomato, Figure 3.4) will help correlate cortical dynein patches with individual nuclear oscillations, and understand why highly processive mutants cause a reduction in nuclear oscillations.

HU-arrested cells give a static representation of the dynein pathway. To better characterize how MTBD mutants effect dynein function, we have prepared strains that will allow us to follow the dynein pathway over an entire cell cycle. We have constructed versions of the strain shown in Figure 3.4 (fluorescently tagged dynein, SPB, and tubulin) with high processivity MTBD mutations introduced. In future experiments we will use alpha factor to synchronize cells at the G1/S transition, and follow cells across an entire cell cycle. This will allow us to look at all aspects of dynein function in yeast, from early Bud6-dependent spindle migration to the site of budding (21), its initial asymmetric cortical localization (spindle retention), and later symmetric cortical localization (spindle elongation) (22). We will be able to determine the onset, duration, and possible deviations from WT behavior for each event.

In vitro motility assays, in which dynein is applied to immobilized MTs (see Chapter 2), are useful to characterize dynein mutants; however, they are not completely reflective of dynein's employment in yeast, where it is offloaded from the plus ends of microtubules to the cell cortex. There, anchored to the cortical membrane protein Num1, it is thought to pull on astral microtubules as a ensemble of approximately 6 dynein molecules (28). It is possible that the collective behavior of dynein ensembles is perturbed in our reported MTBD mutants. In order to address this possibility, we will perform MT gliding assays with MTBD mutants. In these assays dynein is immobilized to a substrate at various concentrations and MTs are added. Akin to 
cortical dynein-MT interactions, dynein pulls on the MTs and causes them to glide over the dynein coated surface. This system will serve as an approximation of the dynein-cortex interaction, and will indicate whether MTBD mutants have altered ensemble properties. It might help explain why, for example, cells expressing the double mutant E3107K/E3197K have nuclear oscillation dynamics similar to WT cells. It is possible that extremely processive dyneins begin to interfere with each other when they are assembled in patches, and thus abrogate the enhanced processivity each individual, isolated dynein would display.

In order to connect MT gliding assays to extensive in vivo characterization of MTBD mutants, we would like to gain more insight into the structural characteristics of the MT-cortex interaction. In WT yeast cells, microtubules often interact end-on with the cell cortex, and appear to be coupled to depolymerization (14). In cells lacking dynein the rate of MT shrinking is diminished by half (14), a result that was recapitulated in a recent in vitro study (29). A new technique offers a possibility to observe these cortical attachments in situ. Cryogenic focused ion beam milling combined with cryo-electron tomography (cryo-ET), allows the 3D reconstruction of cellular compartments previously not accessible to cryo-ET (30). To this end, we have initiated a collaboration with Elizabeth Villa in the Baumeister lab (Max Planck Institute of Biochemistry). The technique has already been applied to yeast cells with success, allowing the visualization of individual ribosomes (30). If one considers only the dynein heavy chains and the dynein interacting complex, dynactin, the molecular weight of the complex is well in excess of $2 \mathrm{MDa}$. This represents about half the size of a eukaryotic ribosome, and offers the intriguing possibility that dynein-MT interactions will be detected by FIB/Cryo-ET. We hope to meld in 
vivo spindle dynamics, in vitro gliding assays, and 3D reconstructions into a model capable of shedding light on why yeast dynein has been selected for sub-maximal motility. 


\section{References}

1. J. K. Moore et al., Function of dynein in budding yeast: mitotic spindle positioning in a polarized cell, Cell Motil Cytoskeleton 66, 546-555 (2009).

2. D. Eshel et al., Cytoplasmic dynein is required for normal nuclear segregation in yeast, Proc Natl Acad Sci USA 90, 11172-11176 (1993).

3. Y. Li et al., Disruption of mitotic spindle orientation in a yeast dynein mutant, Proc Natl Acad Sci USA 90, 10096-10100 (1993).

4. M. Müller et al., Directional mRNA transport in eukaryotes: lessons from yeast, Cell Mol Life Sci 64, 171-180 (2007).

5. A. Fagarasanu, R. A. Rachubinski, Orchestrating organelle inheritance in Saccharomyces cerevisiae, Curr. Opin. Microbiol. 10, 528-538 (2007).

6. D. Eshel et al., Cytoplasmic dynein is required for normal nuclear segregation in yeast, Proc Natl Acad Sci USA 90, 11172-11176 (1993).

7. S. L. Reck-Peterson et al., Single-molecule analysis of dynein processivity and stepping behavior, Cell 126, 335-348 (2006).

8. W.-L. L. Steven M Markus, Microtubule-dependent path to the cell cortex for cytoplasmic dynein in mitotic spindle orientation, Bioarchitecture 1, 209 (2011). 
9. J.K. Moore et al., Coordinating mitosis with cell polarity: Molecular motors at the cell cortex, Cell Motil Cytoskeleton 66, 546-555 (2009)

10. S. L. Jaspersen, M. Winey, The budding yeast spindle pole body: structure, duplication, and function, Annu Rev Cell Dev Biol 20, 1-28 (2004).

11. L. G. B Byers, Behavior of spindles and spindle plaques in the cell cycle and conjugation of Saccharomyces cerevisiae, J Bacteriol 124, 511 (1975).

12. C. L. Asbury et al., The Dam1 kinetochore complex harnesses microtubule dynamics to produce force and movement, Proc Natl Acad Sci USA 103, 9873-9878 (2006).

13. T. Mitchison, M. Kirschner, Dynamic instability of microtubule growth, Nature 312, 237 242 (1984).

14. J. L. Carminati, Microtubules Orient the Mitotic Spindle in Yeast through Dynein-dependent Interactions with the Cell Cortex, J Cell Biol 138, 629-641 (1997).

15. R. A. Heil-Chapdelaine et al., The Cortical Protein Num1p Is Essential for DyneinDependent Interactions of Microtubules with the Cortex, J Cell Biol 151, 1337-1344 (2000).

16. X. Tang et al., A novel patch assembly domain in Num1 mediates dynein anchoring at the cortex during spindle positioning, J Cell Biol 196, 743-756 (2012).

17. B. Sheeman et al., Determinants of S. cerevisiae dynein localization and activation: implications for the mechanism of spindle positioning, Curr Biol 13, 364-372 (2003). 
18. J.K. Moore el al., Function of Dynein in Budding Yeast: Mitotic Spindle Positioning in a Polarized Cell, Cell Motil Cytoskeleton 66, 546 (2009).

19. A. Burakov et al., Centrosome positioning in interphase cells, J Cell Biol 162, 963-969 (2003).

20. D. L. Beach et al., The role of the proteins Kar9 and Myo2 in orienting the mitotic spindle of budding yeast, Curr Biol 10, 1497-1506 (2000).

21. R. Hoopen et al., Mechanism for Astral Microtubule Capture by Cortical Bud6p Priming Spindle Polarity in S. cerevisiae, Current Biology 22, 1075-1083 (2012).

22. S. Grava et al., Asymmetric recruitment of dynein to spindle poles and microtubules promotes proper spindle orientation in yeast, Developmental cell 10, 425-439 (2006).

23. J. K. Moore et al., Neurodegeneration mutations in dynactin impair dynein-dependent nuclear migration, Proc Natl Acad Sci USA 106, 5147-5152 (2009).

24. M. D. Stuchell-Brereton et al., Functional interaction between dynein light chain and intermediate chain is required for mitotic spindle positioning, Mol Biol Cell 22, 2690-2701 (2011).

25. J. M. Kollman et al., Microtubule nucleating $\gamma$-TuSC assembles structures with 13-fold microtubule-like symmetry, J Cell Biol 466, 879-882 (2010).

26. D. A. Lewis, L. F. Bisson, The HXT1 gene product of Saccharomyces cerevisiae is a new member of the family of hexose transporters, Mol Cell Biol 11, 3804-3813 (1991). 
27. R. K. Miller et al., The cortical localization of the microtubule orientation protein, Kar9p, is dependent upon actin and proteins required for polarization, J Cell Biol 144, 963-975 (1999).

28. S.M. Markus et al., Quantitative Analysis of Pac1/LIS1-mediated Dynein Targeting: Implications for Regulation of Dynein Activity in Budding Yeast, Cytoskeleton (Hoboken) 68, 157 (2011).

29. L. Laan et al., Cortical Dynein Controls Microtubule Dynamics to Generate Pulling Forces that Position Microtubule Asters, Cell 148, 502-514 (2012).

30. A. Rigort et al., Focused ion beam micromachining of eukaryotic cells for cryoelectron tomography, Proc Natl Acad Sci USA 109, 4449-4454 (2012). 
Chapter 4

Discussion and Future Directions

William Bret Redwine 


\section{Initiation of our SRS-MTBD reconstruction efforts}

Chronologically, our cryo-EM studies with dynein did not start with the MTBD, but rather with the one of the largest constructs, the entire dimeric motor domain (MW $663 \mathrm{kDa}$, See Appendix 2). We were encouraged to find we could both isolate the protein to high purity and yield, as well as obtain highly decorate microtubules under cryogenic conditions. However, a recent extension of earlier work on dynein motility $(1)$ demonstrated that dynein stepping is highly variable, suggesting that dimers might not bind with preferred orientations on the microtubule lattice (2). This was reflected in our attempts to obtain 2D averages of dimers bound to microtubules. Focusing on the sides of microtubules, where we reasoned that the heads of dynein would be visible, we were unable to obtain meaningful averages (data not shown).

We then considered whether a 2D approach might be more feasible. This $2 \mathrm{D}$ approach was to be centered around collecting images of microtubules sparsely decorated with dimeric dynein constructs. The individual dimers, many thousands of them, would then be subjected to 2D alignment and classification. We would then model different head-head orientations computationally and match their projections to the $2 \mathrm{D}$ class averages. This approach is still potentially informative, as we still lack structural information regarding the orientation of two heads within a dimer. However, a challenge we have observed towards obtaining sparsely decorated microtubules was actually how we began work with SRS-MTBD constructs. We have repeatedly observed that dynein tends to decorate microtubules in an "all-or-nothing" manner (see Figure A2.4); that is, we often observe within the same field of view, microtubules that highly decorated alongside microtubules that lack any visible decoration. During grid preparation, we first absorb microtubules before multiple additions of concentrated dynein 
sample (Appendix 2). This means that all microtubules "see" the dynein sample simultaneously, and suggests that the "all-or-nothing" phenomenon reflects cooperative microtubule binding by dynein. To circumvent this problem, we initially purified the high affinity SRS-MTBD as a means to compete with dimeric dynein for binding sites, and thus, with the correct ratio of dynein to SRS-MTBD, obtain sparse decoration. During this time (2008-2010) several cryo-EM reconstructions of microtubule-bound proteins began to reach resolutions under $10 \AA$, the point at which alpha helices become visible as distinct entities (3-7). At sub-nanometer resolutions, fitting of available crystal structures provides a means to extend the usefulness of cryo-EM reconstructions, and gain considerable mechanistic insight. Encouraged by this possibility, and the crystal structure of a low affinity SRS-MTBD (8), we decided to switch gears and focus our efforts on a high resolution reconstruction of the SRS-MTBD bound to microtubules.

$3 D$ reconstruction of the $M T B D-M T$ complex reveals the basis for bidirectional communication

Our work began with cryo-EM data collection, and a sub-nanometer resolution reconstruction of the a high affinity form of MTBD of dynein bound to its native track, the microtubule. The construct we used was based on a series of constructs designed to alter the registry of dynein's antiparallel coiled-coil stalk. Fixing the stalk in various registries can modulate the affinity of the globular MTBD for microtubules, which forms the basis of the helix sliding hypothesis (9). This hypothesis has seen additional support from cross-linking studies with full length monomeric dynein from $D$. discoideum, which demonstrated that several registries are explored by the motor in solution. Furthermore, fixing the full length motor in a particular registry essentially blocks communication between the MTBD and the ATPase 
subunits in the head (10). Within this framework, whereby the MTBD mediates bidirectional communication between the microtubule and the head, our work uncovered the rearrangement of the helices in the MTBD responsible for microtubule sensing. When we compared our pseudoatomic model of the high affinity MTBD to the low affinity crystal structure, we observed a large displacement of $\mathrm{H} 1$ to a new position stabilized by salt bridges with an acidic patch on $\beta$ tubulin. Due to H1's direct connection to $\mathrm{CC} 1$ in the stalk, we offer the explanation that changing the position of $\mathrm{H} 1$ alters the distribution of registries the stalk can explore. In the low affinity state, the $\alpha$-registry is unlikely to occur, simply because the compact MTBD prevents the upward (head-direction) movement of CC1 necessary to accommodate the $\alpha$-registry. In the high affinity state, the upward reposition of $\mathrm{H} 1$ would disfavor the low affinity $\beta$-registry.

\section{Uncoupling the head-MTBD bidirectional communication pathway}

One testable prediction of our model is that if we can engineer constructs to adopt a high affinity $\mathrm{H} 1$ conformation independent of stalk registry, we would be able to uncouple the MTBD from the head. In order to test this prediction we envision two approaches (Figure 4.1): 1) moving $\mathrm{H} 1$ along the path of its low to high affinity conformational change, and 2) introducing flexibility that would allow $\mathrm{H} 1$ to adopt favorable interactions with the microtubule. In the first approach, which we refer to as "treadmilling", we will remove residues from the loop preceding H1 (CC1-H1 loop) and place them immediately following H1 (H1-H2 loop). In this way, the position of $\mathrm{H} 1$ would move by small degrees towards the high affinity conformation. The RMSD between the low and high affinity $\mathrm{H} 1$ conformations is $10.1 \AA$. The loop between CC1 and $\mathrm{H} 1$ contains two residues that are suitable for translocation to the $\mathrm{H} 1-\mathrm{H} 2$ loop. Since the 


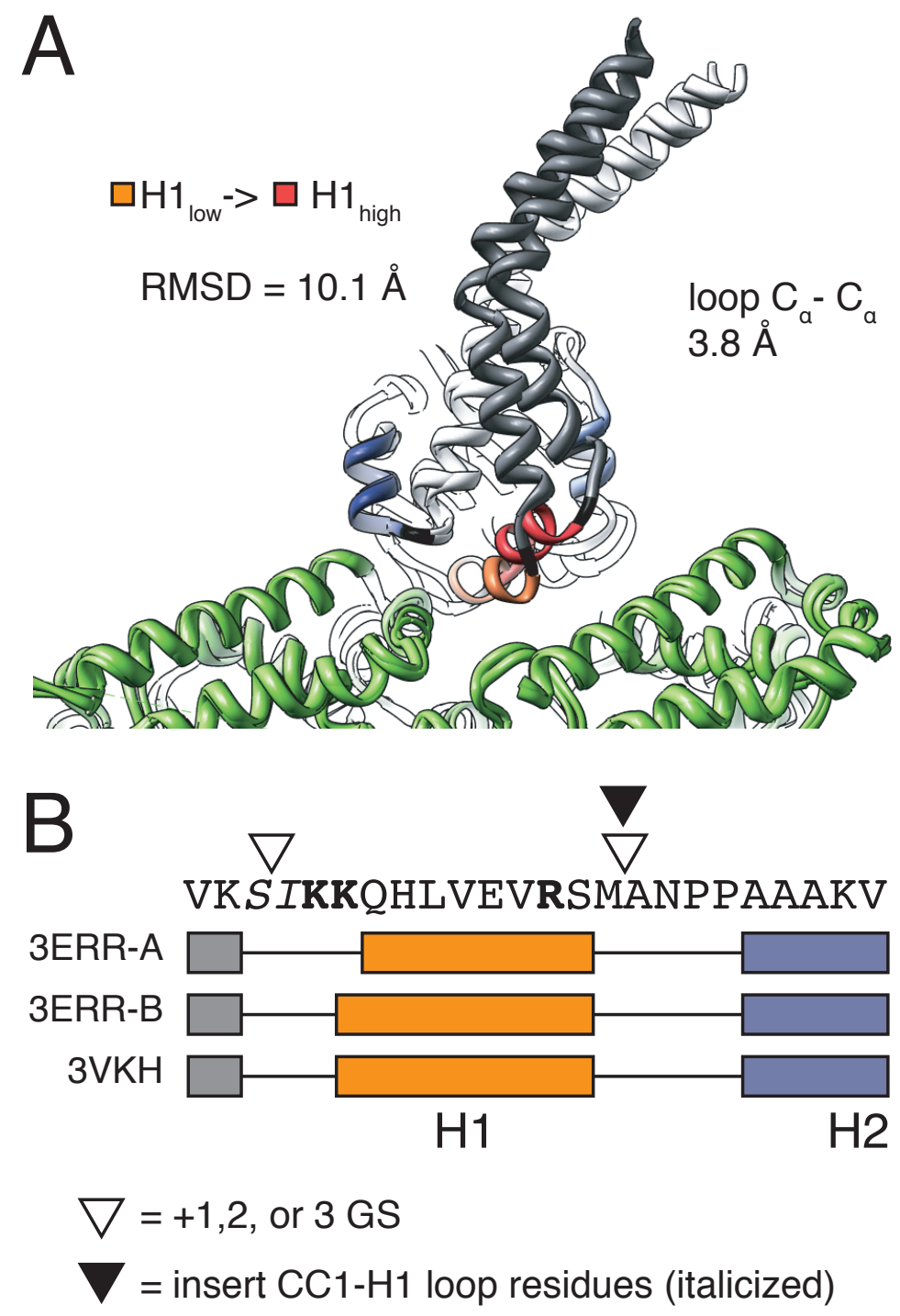

Figure 4.1. Uncoupling the head-MTBD bidirectional communication pathway. (A) Overlay of the low (orange) and high (red) affinity MTBD (see Chapter 2 for details). (B) Helix and loop assignments from available MTBD crystal structures: $\mathrm{CC} 1$ (grey), $\mathrm{H} 1$ (orange/red), $\mathrm{H} 2$ (light blue). The insertion point for glycineserine (GS) linkers (white triangle) is indicated. The point of insertion for CC1$\mathrm{H} 1$ residues (italics) is indicated (black triangle). Residues that form salt bridges with the microtubule are indicated (bold). Tubulin is colored green. 
distance between $\mathrm{C}_{\alpha}$ carbons is $3.8 \AA$, this represents a potential net movement of $7.6 \AA$ towards the high affinity binding site, plus added length to the H1-H2 loop. We will use a low affinity SRS-MTBD fusion (26:19, corresponding the reported crystal structure (8)), and determine if the affinity for microtubules is increased by "treadmilling". In the second approach we will introduce flexibility by inserting glycine-serine linkers (1X Gly-Ser - 3X Gly-Ser, singly and in all combinations) into the $\mathrm{CC} 1-\mathrm{H} 1$ and $\mathrm{H} 1-\mathrm{H} 2$ loops. Again we will address, with low affinity SRS-MTBD constructs, whether microtubule binding affinity increases. For both approaches, if a given construct binds to microtubules with increased affinity, we will make the equivalent alterations to full length monomeric dynein. Given that bidirectional communication might be impacted by changing how $\mathrm{H} 1$ responds to cues form the ring, we will test whether microtubule release is affected with a single molecule release assay (19). In this assay, dynein is bound to microtubules under no nucleotide conditions and imaged as single molecules by TIRF microscopy. Using a homemade flow chamber, the solution will be replaced by buffer containing ATP. Under these conditions WT monomeric dynein rapidly releases from microtubules, with subsequent rebinding events being brief and corresponding to a single ATP turnover event. If a construct is defective for bidirectional communication, we would expect it to persists for extended periods of time on the microtubule.

Is the MTBD a self-contained, reversible microtubule sensor?

The SRS-MTBD constructs have been extremely useful for understanding the molecular basis for microtubule binding by dynein $(8,9)$, but they are fixed constructs that force the MTBD to adopt different conformations. A minimal, unconstrained MTBD from S. cerevisiae (11) 
offers the unique opportunity to determine the conformation of an unconstrained construct in solution and bound to microtubules, and therefore study the dynamics of the MTBD. The appealing aspect of this construct is that while it contains no fusion protein sequence to stabilize a particular registry of the stalk, it exhibits high affinity for microtubules. We have designed a very similar version of this construct, which also exhibits high affinity for microtubules. In order to determine the conformation of this construct bound to microtubules, we have collected a cryoEM dataset (see Appendix 1 for details), and are currently pursuing a high resolution reconstruction of this construct. In order to determine the conformation of this construct in solution we will pursue two strategies: x-ray crystallography and determination of the registry by biochemical methods. X-ray crystallography has potential, given we can express and purify this construct to high purity and yield (Appendix 1), and structure determination will be aided greatly by the use of previous structures as search models for molecular replacement. In the biochemical approach (Figure 4.2), we will place cysteines within the stalk of the ScMTBD such that they form only within the context of specific registries ( $\alpha=$ high affinity, and $\beta+=$ low affinity), based upon the strategy reported for full length monomeric dynein (10). We will perform crosslinking experiments to test which registry is formed in solution and in the presence of microtubules. Since the dynein-microtubule interaction is extremely salt sensitive (Figure 2.10), we will reverse binding by increasing the ionic strength in samples of ScMTBD bound to microtubules and determine whether the registry remains in the microtubule-bound configuration, or if it collapses back to the low affinity ground state.

$3 D$ reconstruction of full length dynein via an incremental approach 

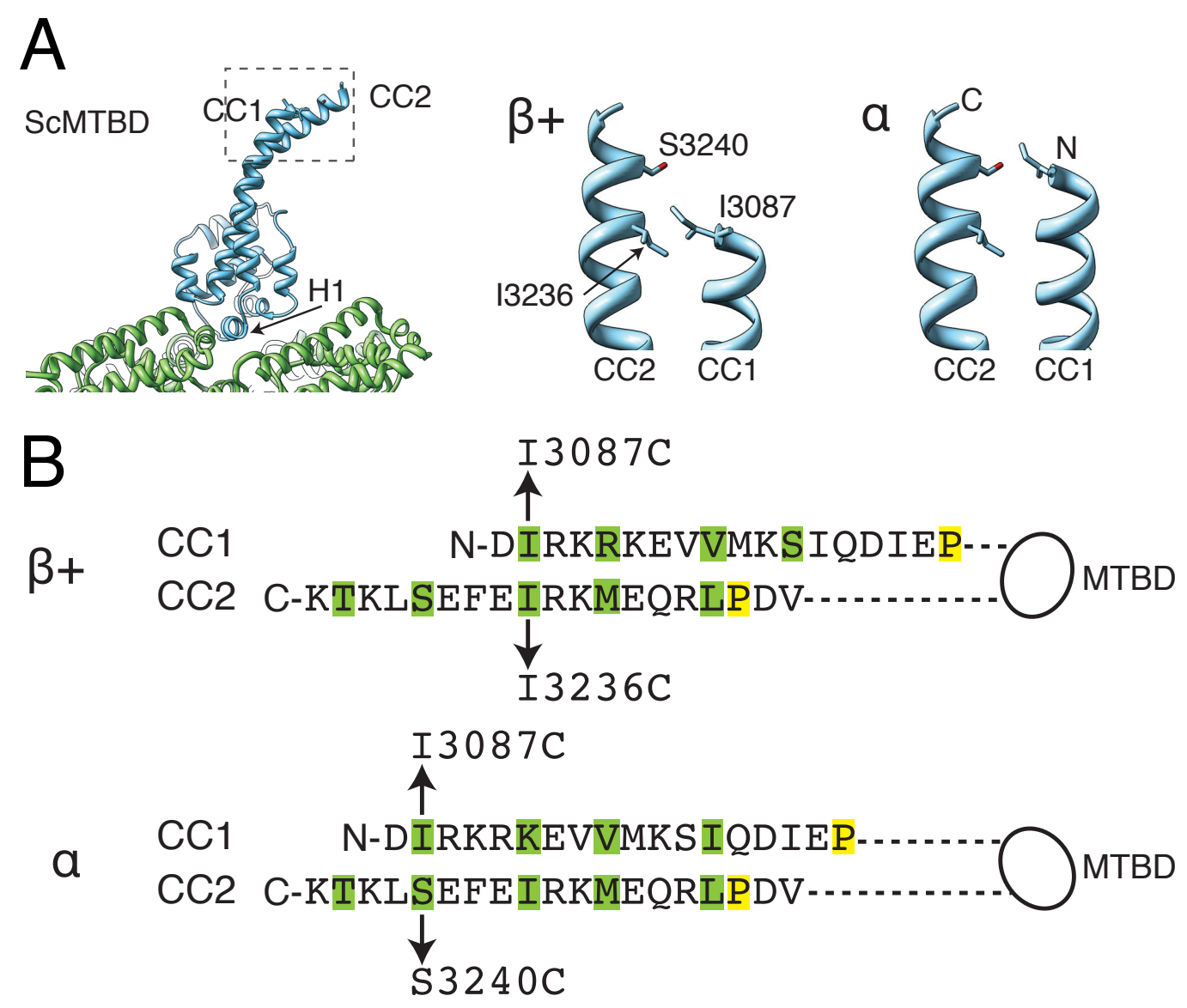

Figure 4.2. Determining if the MTBD is a self-contained, reversible microtubule sensor. (A) Left: homology model of the MTBD from S. cerevisiae (ScMTBD, blue, generated with SWISS-MODEL server) bound to tubulin (green). Area enlarged in middle and right panels is indicated with a dashed rectangle. Middle: $\beta+$ registry with residues of interest indicated. Right: predicted $\alpha$ registry with $\mathrm{N}$ and $\mathrm{C}$ termini labeled. Residues are labelled in middle panel, the $\mathrm{N}$ - and $\mathrm{C}$ termini are indicated in right panel. Note the 4 residue shift of I3087, which switches its hydrophobic interaction partner from I3236 to S3240 (B) Crosslinking strategy to fix the registry of the ScMTBD in the $\beta+$ (top) and $\alpha$ (bottom) configuration. $\mathrm{N}$ - and $\mathrm{C}$ - termini, and the location of cysteine mutants are indicated. Hydrophobic residues corresponding to "a" and " $d$ " locations of the heptad repeat are colored green. Absolutely conserved proline residues are colored yellow. 
Our high resolution MTBD reconstruction forms the base for future reconstructions of ever more complicated dynein constructs. The various dynein constructs we now have at our disposal, including monomeric and dimeric dynein, lend themselves to an incremental structural approach. Admittedly, we anticipate diminishing attainable resolutions as the size of the construct increases. Our work with the SRS-MTBD suggests that the further away from the microtubule surface, the less likely sufficient structural order will be present for sub-nanometer resolution reconstructions. This is supported by a low resolution reconstructions of a 12-heptad repeat SRS-MTBD reconstruction reported previously (8), monomeric dynein construct (12), and even high resolution reconstructions of modestly size structures (e.g. Ndc80) (6). While a subnanometer reconstruction of the MTBD proved necessary to gain new mechanistic insight, the threshold for what constitutes meaningful resolution is likely to decrease significantly as larger dynein constructs are analyzed. Crystal structures $(13,14)$ and cryo-EM reconstructions $(15)$ have revealed the conformation of the linker present in free dynein constructs, but the linker conformation of microtubule-bound dynein is not currently known. The linker can be resolved at resolutions near $20 \AA$, which is a reasonable goal for reconstructions of monomeric dynein bound to microtubules. With regards to dimeric dynein, an outstanding question regards the orientation of the two heads relative to each other when bound to microtubules. It is possible that this could be addressed by methods that do not rely on 3D reconstructions. One could collect images of microtubules sparsely decorated with dimeric dynein and perform 2D alignment and classification. These class averages could then be matched to simulated conformations of dynein dimers modeled with x-ray crystal structures and the relative orientation of the heads could be determined. 
Once the conformation of monomeric and dimeric dynein on microtubules is determined, it will be important to address how dynein's many co-factors affect its conformation (16). Two factors of immediate interest are Lis1 (Pac1 in yeast) and the mutisubunit complex dynactin, both of which have been shown to alter dynein's motility properties $(17,18)$ (J. Huang and A. Roberts, et al. in press). Lis1 appears to be necessary for dynein function under high loads (17), and recently has been shown to constitute a molecular "clutch" that uncouples dynein's ATPase and microtubule binding activities (19). Dynactin is a ubiquitous dynein co-factor in vivo (20), and has been shown to increase dynein processivity approximately 2-fold (18). Do co-factors change overall orientation of monomers within the dimer? What happens to dynein's various elements (linker, buttress, stalk, MTBD) while in complex with these co-factors? Is the linker conformation altered? Does the stalk angle, and therefore dynein's force vector, change? Provided we can obtain sufficient resolution, do these factors alter the conformation of the MTBD through allosteric effects? While Lis1 exerts its effects on the core motor domain, and is therefore immediately addressable by cryo-EM with "mini-dimers" (Appendix 2), dynactin binds to the tail of full length dynein. Based on the experience of the Reck-Peterson lab, dynactin and full length dynein are both difficult to purify from yeast to the yield and purity necessary for cryo-EM. Therefore, some investment in alternative expression and purification systems will be necessary.

\section{Processivity in the context of multiple motors}

Perhaps the most surprising result of our work is that dynein is an inherently restrained motor. Under what we consider standard motility conditions ( $\sim 80 \mathrm{mM}$ total ionic strength), 
mutations that disrupt dynamic salt bridges at $\mathrm{CC} 1-\mathrm{H} 1$ and $\mathrm{H} 6$ resulted in run length increases of 5 and 6 fold respectively. More dramatically, the combination of these mutations cannot be analyzed under standard conditions, as the beginnings and ends of runs are often missing from data. Under higher ionic strength conditions (130 $\mathrm{mM}$ total ionic strength) that effectively abolish WT dynein motility, the double mutant has a run length that is 3.5-fold greater than the highest single mutation. Extrapolated to standard conditions, this combination of mutations increases dynein's run length 21 fold over wild type, to approximately $42 \mu \mathrm{m}$. To gain these mutations S. cerevisiae would require only single nucleotide changes at each codon (GAG -> AAG), indicating a strong selective pressure not to accumulate them.

Why then is dynein intrinsically an under-performer? In Chapter 3 we present preliminary evidence that under specific conditions, dynein-driven cellular processes are impaired when dynein is highly processive. An important function of many eukaryotic dyneins that is not observable in S. cerevisiae is intracellular transport. In S. cerevisiae dynein has no known cargo-bearing responsibilities, and is solely utilized for mitotic spindle movements. In filamentous fungi, such as $A$. nidulans, dynein has diverse cargo-bearing roles, including the movement of endosomes and peroxisomes (21). It will be interesting to use this model organism to determine if fine-tuned processivity is needed for efficient cargo transport. For example, do cargo that are connected to dynein and kinesin exhibit a skewed distribution towards the minus end of microtubules when dynein is overly processive? Do cells attempt to balance the scales by recruiting more kinesin to these cargo, or do they recruit fewer dynein molecules?

One possible explanation why dynein has been selected for sub-maximal processivity involves the synchronization of multiple motors. Many cargo have multiple motors attached to 
them, including ensembles of dynein and kinesin. Recent unpublished work in our lab has led to the development of synthetic cargo that can be affixed with specific numbers of dynein in defined geometries (N. Derr and B. Goodman, submitted). These synthetic cargo could be used to address how multiple highly processive work together to move along microtubules. When compared to WT assemblies, do they interfere with each other as more motors are added to the cargo? Can we model to behavior of cortical patches of dynein in yeast, and thereby recapitulate defects in dynein-driven movements in vitro?

\section{Concluding remarks}

The work presented here leads many possible avenues for future work. Because this project relied heavily on cryo-EM, the tools applied and developed for this work will be applicable to more biochemically complicated dynein constructs. The biological implications, which we are actively investigating, should provide additional insight into dynein function in cells. Lastly, the highly processive dynein constructs described should prove be valuable tools for future experiments. An immediate application will be towards the reconstruction of monomeric dynein bound to microtubules, where high decoration of the microtubule lattice will be very important. The enhanced affinity of the MTBD mutants should prove useful in obtaining highly decorated microtubules. 


\section{References}

1. S. L. Reck-Peterson et al., Single-molecule analysis of dynein processivity and stepping behavior, Cell 126, 335-348 (2006).

2. W. Qiu et al., Dynein achieves processive motion using both stochastic and coordinated stepping, Nat Struct Mol Biol 19, 193-200 (2012).

3. C. V. Sindelar, K. H. Downing, The beginning of kinesin's force-generating cycle visualized at 9-A resolution, J Cell Biol 177, 377-385 (2007).

4. C. V. Sindelar, K. H. Downing, An atomic-level mechanism for activation of the kinesin molecular motors, Proc Natl Acad Sci USA 107, 4111 (2010).

5. F. J. Fourniol et al., Template-free 13-protofilament microtubule-MAP assembly visualized at 8 A resolution, $J$ Cell Biol 191, 463-470 (2010).

6. G. M. Alushin et al., The Ndc80 kinetochore complex forms oligomeric arrays along microtubules, Nature 467, 805-810 (2010).

7. A. J. Bodey, M. Kikkawa, C. A. Moores, 9-Angström structure of a microtubule-bound mitotic motor, J Mol Biol 388, 218-224 (2009).

8. A. P. Carter et al., Structure and functional role of dynein's microtubule-binding domain, Science 322, 1691-1695 (2008).

9. I. R. Gibbons et al., The affinity of the dynein microtubule-binding domain is modulated by the conformation of its coiled-coil stalk, J Biol Chem 280, 23960-23965 (2005). 
10. T. Kon et al., Helix sliding in the stalk coiled coil of dynein couples ATPase and microtubule binding, Nat Struct Mol Biol 16, 325-333 (2009).

11. N. Mizuno et al., Dynein and kinesin share an overlapping microtubule-binding site, EMBO J 23, 2459-2467 (2004).

12. N. Mizuno et al., Three-dimensional structure of cytoplasmic dynein bound to microtubules, Proc Natl Acad Sci USA 104, 20832-20837 (2007).

13. T. Kon et al., The $2.8 \AA$ crystal structure of the dynein motor domain, Nature 484, 345-350 (2012).

14. H. Schmidt, E. S. Gleave, A. P. Carter, Insights into dynein motor domain function from a 3.3-Å crystal structure, Nat Struct Mol Biol 19, 492-497 (2012).

15. A. J. Roberts et al., ATP-Driven Remodeling of the Linker Domain in the Dynein Motor, Structure 20, 1670-1680 (2012).

16. J. R. Kardon, R. D. Vale, Regulators of the cytoplasmic dynein motor, Nat Rev Mol Cell Biol 10, 854-865 (2009).

17. R. J. Mckenney, M. Vershinin, A. Kunwar, R. B. Vallee, S. P. Gross, LIS1 and NudE induce a persistent dynein force-producing state, Cell 141, 304-314 (2010).

18. J. R. Kardon et al., Regulation of the processivity and intracellular localization of Saccharomyces cerevisiae dynein by dynactin, Proc Natl Acad Sci USA 106, 5669-5674 (2009). 
19. J. Huang and A. Roberts et al., Lis1 acts as a "clutch" between the ATPase and microtubulebinding domains of the dynein motor, Cell 150, 975-986 (2012).

20. T. A. Schroer, Dynactin, Annu Rev Cell Dev Biol 20, 759-779 (2004).

21. M. J. Egan, K. Tan, S. L. Reck-Peterson, Lis1 is an initiation factor for dynein-driven organelle transport, J Cell Biol 197, 971-982 (2012). 


\section{Appendix 1}

Preparation and data collection of a minimal MTBD from Sacharomyces cerevisiae

William Redwine, Rogelio Hernandez-Lopez, and Andres Leschziner 


\section{Contributions}

William Redwine performed biochemistry and electron microscopy data collection.

Rogelio Hernandez-Lopez and William Redwine performed image processing. Andres Leschziner provided mentorship. 


\section{Introduction}

Structure determination of proteins bound to microtubules presents special challenges. Primarily this stems from the fact that microtubules are tubular 2D crystals that extend to great lengths, and cannot form the contacts necessary for 3D crystals. Ideally, high resolution structures of MT interacting proteins in complex with microtubules can be approached from a hybrid method that incorporates X-ray crystallography of the free MT-binding protein of interest, and cryo-EM reconstructions of the protein bound to MTs. The strength of this approach, when feasible, can be found in recent single-particle reconstruction approaches of MT bound proteins have pushed the reconstructions to sub-nanometer resolutions $(<10 \AA)$. At or below this resolution it is possible to directly visualize alpha helices and beta sheets, allowing the accurate fitting of crystal structures.

In Chapter 2, we presented a sub-nanometer resolution reconstruction of the high affinity SRS-MTBD construct bound to MTs. This reconstruction, and its subsequent pseudoatomic model provided insights into the mechanism by which dynein binds and releases MTs. While the SRS-MTBD constructs have proven to be valuable resources towards understanding the mechanism dynein uses to bind and release MTs, admittedly they are engineered constructs that force dynein into a particular registry. It is clear that in the context of a full length motor the stalk explores multiple registries in solution, and that the registries dictated by the SRS constructs, though visited, are not fixed (1). An important unresolved question, which is suggested by our work (Chapter 2), is the degree to which the MT directly causes changes in the conformation of the MTBD. 
Ideally, we would determine the free and MT-bound structures of a construct that responds conformationally to the presence of MTs. One reported construct has this potential. It consists of the MTBD and distal stalk from S. cerevisiae (3). Although this construct (referred to here as ScMTBD) is of unconstrained registry, it exhibits high affinity for MTs (3). Other unconstrained MTBD constructs reported thus far exhibit a low microtubule affinity conformation in solution (4) (PDB 2RR7, unpublished). Thus, it is possible that the ScMTBD adopts a low affinity conformation in solution, and undergoes conformational changes upon binding to microtubules. As we know that the SRS portion of our SRS-MTBD cryo-EM reconstruction is of poorer resolution than the portion of the map encompassing the MT and MTBD (see Figure 2.3), it is reasonable to assume that a smaller construct like the ScMTBD (19 $\mathrm{kDa}$ ), with fewer flexible parts, would yield a higher resolution structure. Given its small size, it is also an attractive candidate for $\mathrm{x}$-ray crystallography. Together, higher high resolution structures of the free and MT-bound forms would represent the first such pair.

We had the unique opportunity to send samples of the minimal ScMTBD bound to MTs directly to the manufacturer of our microscopes, FEI, to acquire data on their newest microscope, the Titan Krios. Here, we report out efforts to optimize the purification of ScMTBD and collect high quality cryo-EM data. 


\section{Materials and methods}

The DNA sequence encoding a minimal MTBD (amino acids 3086-3244, referred to hereafter as ScMTBD) from S. cerevisiae DYN1 was cloned into pMAL-C2 by the CPEC cloning method (4). The forward primer used to amplify the MTBD was designed to include sequence encoding an N-terminal PreScission Protease cleavage placed between MBP and the MTBD. BL21 DE3 (pLysS) cells transformed with the plasmid were grown in 8 L of LB broth at $37 \mathrm{C}$ to an optical density at $600 \mathrm{~nm}$ of 0.5 . The cells were chilled on ice with periodic mixing until the temperature was $16^{\circ} \mathrm{C}$. Protein expression was induced with $0.5 \mathrm{mM} \beta$-Dthiogalactopyranoside and the cells were further incubated at $16{ }^{\circ} \mathrm{C}$ for $6 \mathrm{~h}$. All subsequent steps were performed at $4{ }^{\circ} \mathrm{C}$ or on ice. Cells were harvested by centrifugation and were resuspended in $70 \mathrm{~mL}$ lysis buffer $(20 \mathrm{mM}$ Tris- $\mathrm{HCl}, \mathrm{pH} 8.0 ; 150 \mathrm{mM}$ KOAc, $1 \mathrm{mM}$ EDTA, $2 \mathrm{mM} \mathrm{MgOAc}$, 10\% glycerol, $20 \mathrm{mM}$ imidazole, $5 \mathrm{mM} \beta$-mercaptoethanol, $1 \mathrm{mM} \mathrm{PMSF}$, and $1 \mu \mathrm{g} / \mathrm{mL}$ each pepstatin A, aprotinin, benzamidine, and leupeptin). Lysozyme was added to a final concentration of $0.1 \mathrm{mg} / \mathrm{mL}$, and the cells were placed on ice for 15 minutes. The cells were lysed by sonication ( 8 continuous pulses at $80 \%$ power, with 2 minutes between pulses) and the resulting lysate was centrifuged at $120,000 \mathrm{x}$ g in a Ti45 rotor (Beckman) for $30 \mathrm{~min}$ at $4^{\circ} \mathrm{C}$. The cleared lysate was applied to a $30 \mathrm{~mL}$ amylose agarose (New England Biolabs) column by gravity at a flow rate of $\sim 1 \mathrm{~mL} / \mathrm{min}$. The column was then washed with $400 \mathrm{~mL}$ lysis buffer by gravity. Bound protein was eluted with lysis buffer containing $20 \mathrm{mM}$ maltose. Peak fractions containing the ScMTBD fusion, as determined by SDS-PAGE analysis, were pooled, dialyzed for 4 hours against lysis buffer, and concentrated $\sim 18 \mathrm{mg} / \mathrm{mL}$ with Ultracell concentration columns with a molecular weight cut-off of $30 \mathrm{kDa}$ (Millipore). Next, PreScission Protease was 
added at a wt:wt ratio of 1:500, and the reaction was incubated overnight on ice. ScMTBD was partially separated from MBP and MBP-ScMTBD on an S75 16/60 gel filtration column. To further purify the ScMTBD, S75 fractions $(9 \mathrm{~mL})$ enriched for the ScMTBD were diluted in lysis buffer without added salt (low salt buffer $=$ lysis buffer lacking KOAc) to reduce the salt concentration to $50 \mathrm{mM}$. This sample was applied to a Resource $\mathrm{S}$ ion exchange column, washed in low salt buffer, and eluted over a a linear $\mathrm{NaCl}$ gradient from 0 to $1 \mathrm{M} \mathrm{NaCl}$. ScMTBD eluted at approximately $350 \mathrm{mM} \mathrm{NaCl}$. The peak fractions were pooled, diluted to reduce the salt concentration to $\sim 150 \mathrm{mM}$, and concentrated to $1.4 \mathrm{mg} / \mathrm{mL}$ with an Ultracell concentrating column with a $10 \mathrm{kDa}$ MWCO filter.

Cryo-EM grids were prepared as described in Appendix 2 with Protochips C-flat 2-2C grids. Data was collected on a Titan Krios electron microscope (FEI) operating at $200 \mathrm{keV}$ and equipped with a 4k x 4k Falcon direct electron detector (FEI). 


\section{Results}

We expressed and purified a minimal MTBD to high purity and yield (Figure A1.1). We used a sequence of steps that included, in order: affinity purification, gel filtration, and ion exchange. As the ion exchange step efficiently separates MBP and the ScMTBD, in the future we would reverse the order of the gel filtration and ion exchange steps. Including gel filtration as the final step ensure that the construct is monodisperse and free from aggregates (although subsequent concentration could create aggregates). At a concentration of 50-60 $\mu \mathrm{M}$ the construct decorates MTs to a high degree, which is evident in the saw-like pattern along the edges of MTs, with the "teeth" constituting individual MTBDs binding at the $8 \mathrm{~nm}$ repeat of the intradimer interface (Figure A1.2).

We screened grids for a batch suitable for shipment to FEI, for the collection of a large dataset on direct detector in a Titan Krios microscope (FEI) (Figure A1.3 B). We processed this dataset using a previously reported alignment and averaging approach (6). Class averages and their power spectra revealed that this new dataset is of excellent quality. The class averages revealed that the ScMTBD in this preparation decorated the MT to a high degree (Figure A1.3 C). Analysis of the power spectra revealed not only the expected $40 \AA$ (tubulin monomer repeats) and $80 \AA$ (ScMTBD repeats) layer lines, but also many higher resolution layer lines (Figure A1.3 D). A faint layer line is visible near $\sim 8.7 \AA$, which is very close to our calculated value of the helical rise of 14 protofilament MTs ( 9.2 A, see Chapter 2, Materials and Methods). 


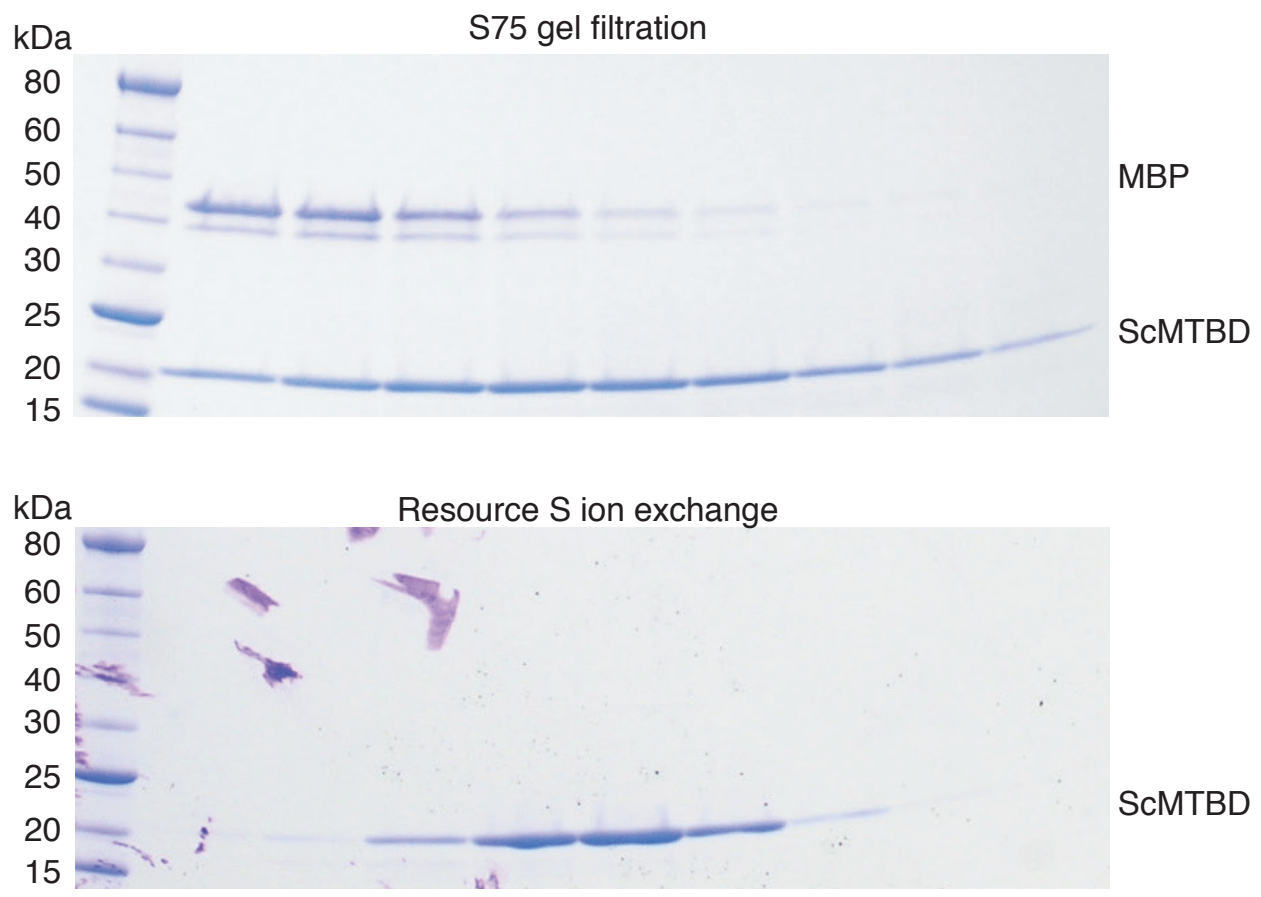

Figure A1.1. Purification of a minimal $S$. cerevisiae MTBD. (top) The peak fractions containing the ScMTBD from S75 gel filtration chromatography. The ScMTBD overlaps with its cleaved fusion partner, MBP. (bottom) Peak fractions containing the ScMTBD from Resource S ion exchange chromatography. MBP, which appears in the flow through fraction, is effectively separated from the ScMTBD. 

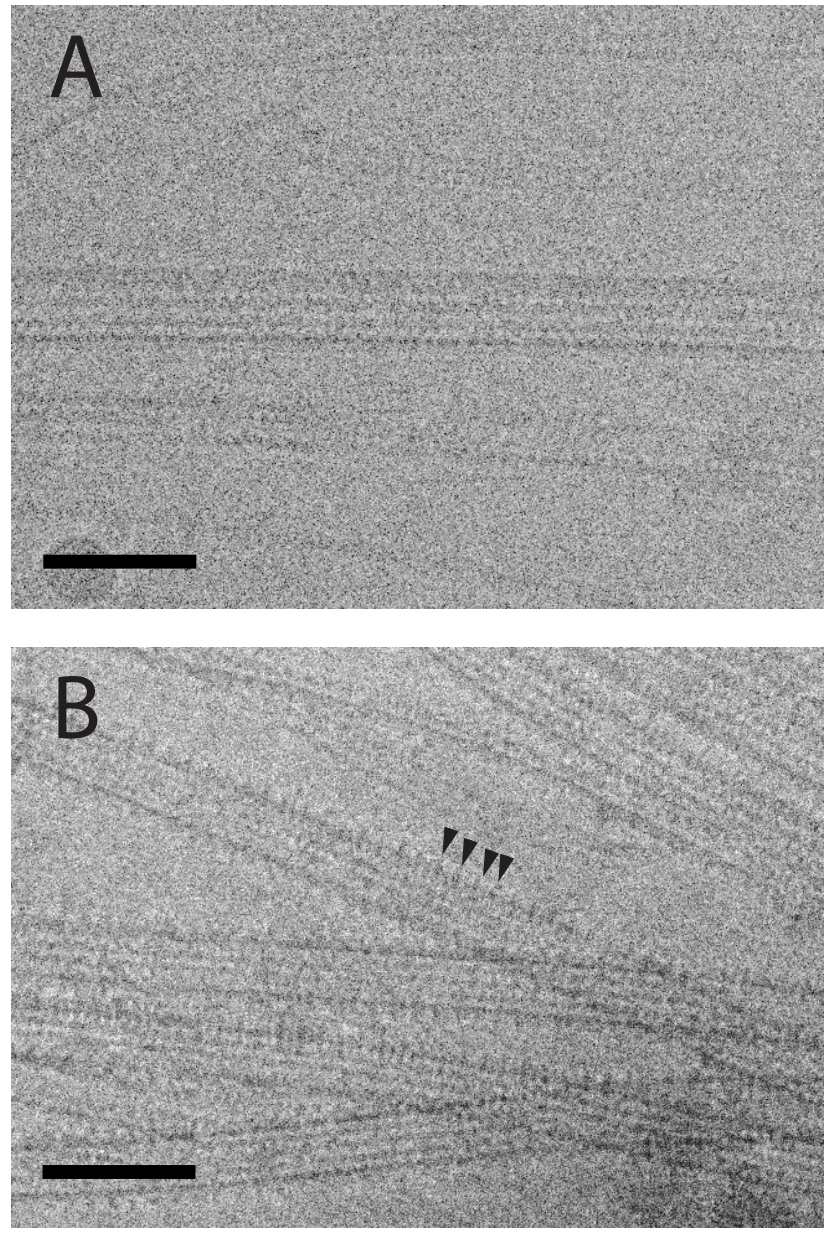

Figure A1.2. Microtubules highly decorated with a minimal MTBD. (A) Section of a micrograph with MTs not incubated with ScMTBD. (B) Section of a micrograph containing MTs incubated with $50 \mu \mathrm{M}$ ScMTBD. Repeated structures are clearly visible on the edges of MTs (arrows). Scale bar is 50 nm. 

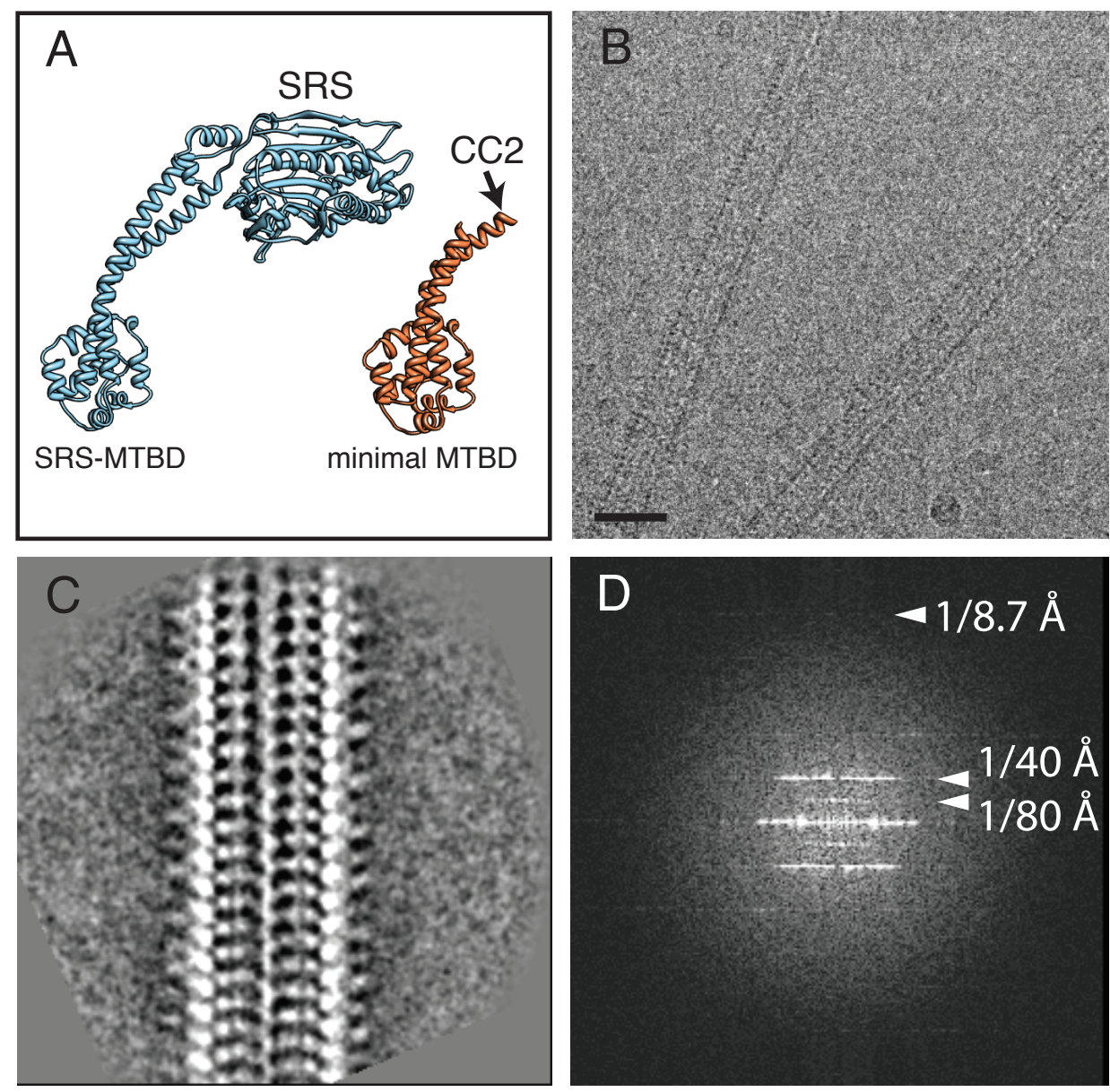

Figure A1.3. High quality ScMTBD data collection on a Titan Krios. (A) Structure of the ScMTBD (orange) modeled from the low affinity SRS-MTBD crystal structure (blue) \{Carter:2008bg\}. Note that CC2 is longer than $\mathrm{CC} 1$, potentially allowing multiple stalk conformations. (B) Segment of a micrograph collected on a direct detector. Scale bar represents $50 \mathrm{~nm}$. Note jagged edge of the MTs, formed by the ScMTBD binding at $8 \mathrm{~nm}$ intervals. (C) Class average of $750 \AA$ segments from 14 protofilament MTs decorated with ScMTBD. (D) Power spectrum of the class average shown in (C). Strong layer lines are present at $40 \AA$ (tubulin monomers) and $80 \AA$ (ScMTBD), as well as higher resolution layer lines. 


\section{Future Directions}

We are currently processing our dataset of the ScMTBD as described in Chapter 2. We anticipate that the resulting reconstructions could surpass the resolution we obtained for the high affinity SRS-MTBD construct. An independent reconstruction will allow us to determine whether the high affinity state adopted by the SRD-MTBD represents a general high affinity conformation.

It is possible that the MT is, in part, responsible for stabilizing specific conformations of the MTBD. In order to fully understand how the MT influences the conformation of the MTBD, it will be important to solve the structure of the ScMTBD in the absence of MTs. In the future we will use a X-ray crystallography to determine the structure of the free ScMTBD. Will will also pursue crosslinking studies to determine whether the ScMTBD is a reversible MT sensor (see Chapter 4 for details). 


\section{References}

1. T. Kon et al., Helix sliding in the stalk coiled coil of dynein couples ATPase and microtubule binding, Nat Struct Mol Biol 16, 325-333 (2009).

2. T. Kon et al., he $2.8 \AA$ crystal structure of the dynein motor domain, Nature $484,345-350$ (2012).

3. N. Mizuno et al., Dynein and kinesin share an overlapping microtubule-binding site, $E M B O J$ 23, 2459-2467 (2004).

4. L. Mcnaughton et al., A Low Affinity Ground State Conformation for the Dynein Microtubule Binding Domain, Journal of Biological Chemistry 285, 15994-16002 (2010).

5. J. Quan et al., Circular Polymerase Extension Cloning of Complex Gene Libraries and Pathways, PLoS ONE 4, 6441-e6441 (2009).

6. V. H. Ramey et al., Ab initio reconstruction of helical samples with heterogeneity, disorder and coexisting symmetries, $J$ Struct Biol 167, 97-105 (2009). 
Appendix 2

Preparation of cytoplasmic dynein for cryo-electron microscopy

William Bret Redwine, Samara-Reck Peterson and Andres Leschziner 


\section{Contributions}

William Redwine performed biochemistry and cyro-electron microscopy. Samara Reck-

Peterson and Andres Leschziner provided mentorship. 


\section{Introduction}

The protein samples used for cryo-EM sample preparation and eventual structure determination have fairly stringent purity and concentration requirements. As the proteins are directly imaged in a vitrified buffer, contaminating proteins, and even soluble aggregates of the target protein, will be imaged as well. Either case can complicate processing and structure determination. As such, one must ensure the protein or complex of interest is of high purity. Cryo-EM sample preparation also requires significantly more sample, both in concentration $(\sim$ 5-10 time more than samples used for negative stain) and total amount. The need for higher total amounts of sample stems from the nature of cryo-EM itself; many grids must often be screened. Ice conditions are often not entirely reproducible, and once a sample is transferred and imaged in the microscope, it is very difficult to retrieve it in a usable form. These combined attributes present unique challenges when one works with samples not previously purified to such high concentration, purity, and yield.

In this appendix we describe a protocol for the purification of milligram amounts of highly pure monomeric and dimeric cytoplasmic dynein from Saccharomyces cerevisiae, for use in the preparation of cryo-EM samples. This protocol was adapted from a previously reported protocol for the preparation of dynein for single molecule motility experiments ( 1 ). In addition to a scale increase at all steps, this protocol includes a modified version of galactose induction of Gall promoter-driven expression tailored reduce the cost. The amounts purified are amenable to additional purification steps, such as ionic exchange or gel filtration chromatography. Following 
the protocol for yeast growth, induction, and protein purification, is a detailed description of the grid making process for microtubules decorated with dynein.

Yeast strains expressing monomeric or the truncated, GST-based dimeric ("minidimer") dynein have been described previously (1). These strains contain a modified genomic locus with the Gal1 promoter in place of the native DYN1 promoter, which confers galactose inducible protein expression. The Reck-Peterson lab has extensively modified dynein with different purification and labeling modules (here we describe the purification via a $\mathrm{ZZ}$ tag, which binds with high affinity to IgG), epitope tags, and fluorescent proteins. The combined experience of the lab suggests that expression of minidimer constructs is similar across different tag configurations, and that the protocol described here is widely applicable.

The Gall promoter is repressed by the presence of glucose in the media. Therefore, after growth of starter cultures in glucose, yeast are typically cultured in raffinose containing media, which derepresses the Gall promoter, before the induction of protein expression with galactose. Raffinose, while an efficient derepressor, has as a major drawback its high cost (9/2012 from Sigma-Aldrich: raffinose $=\sim \$ 1.29 / \mathrm{g}$, glucose $=\sim \$ 0.10$ ). To circumvent this problem, dynein expression has been achieved by the growth of small starter cultures in media with raffinose as the sole carbon source, followed by the inoculation of large cultures containing galactose as the sole carbon source. While this decreases the cost over the extensive use of raffinose, galactose significantly increases the doubling time of yeast relative to growth in media containing an equivalent amount of glucose. To combine the best attributes of glucose (low cost, high growth rates) and galactose (essential for expression) we developed a method that does not require the use of raffinose. In this approach, yeast are continuously cultured in media containing glucose as 
the sole carbon source to the point that the cell density plateaus. While overnight growth of yeast is commonly referred to as stationary phase, it actually represents a phase of growth known as post diauxic shift, at which point the available glucose has been depleted. True stationary phase does not commence form many days after inoculation ( $>5$ days) (2). As such, after approximately 16 hour so of continuous growth, yeast will still rapidly respond to the addition of different carbon sources. In our protocol, after depletion of glucose, galactose is added directly to the saturated culture to simultaneously induce both an increase in cell density, as well as the expression of Gall-driven dynein constructs. Below we describe the utilization of this method for yeast grown in Erlenmeyer flasks. The method is also applicable to yeast growth in fermenters (Figure A2.1).

The following description of growth and purification conditions is presented as a protocol to ease direct copying and use in the laboratory. After the protocol an example of the large scale purified product is presented (Figure A2.2), as well as a sample trace tracking the growth of yeast in a fermenter (Figure A2.1). 


\section{Protocol}

\section{Yeast growth conditions}

1. From freshly grown single colonies start $6 \times 10 \mathrm{~mL}$ cultures of YPD. Incubate overnight with shaking at $30^{\circ} \mathrm{C}$.

2. In the morning transfer each culture to $100 \mathrm{~mL}$ of YPD in a $250 \mathrm{~mL}$ flask. Incubate overnight with shaking at $30{ }^{\circ} \mathrm{C}$.

3. In the morning transfer each $100 \mathrm{~mL}$ culture to $2 \mathrm{~L}$ of $2 \mathrm{xYP}$ with $1 \%$ glucose. Incubate overnight with shaking $(225-250 \mathrm{rpm})$ at $30^{\circ} \mathrm{C}$.

4. In the morning check the $\mathrm{OD}_{600}$ of a couple of flasks. Continue incubating for at least another doubling time ( $\sim 90-120$ minutes). Check the $\mathrm{OD}_{600}$ again. If the yeast have stopped doubling, they can be induced.

5. Induction can be done for any length of time between 6 hours and overnight. To induce add $100 \mathrm{~mL}$ of $40 \%$ galactose per $2 \mathrm{~L}$ media, for a final concentration of $2 \%$.

6. Collect cells by centrifugation in $1 \mathrm{~L}$ bottles at $6000 \mathrm{rpm}$ for 6 minutes. Spin the first $6 \mathrm{~L}$, decant the media, and spin remaining $6 \mathrm{~L}$ on top of the first pellet. 
7. Decant the media, and resuspend the pellets up to $1 \mathrm{~L}$ per bottle in deionized water to wash (vortexing the pellets first in a small amount of water helps). Centrifuge again as above and decant the water.

8a. To freeze the cells scoop up the pellet with a spatula, fill a $60 \mathrm{~mL}$ syringe (without needle attached) and squeeze the cells into liquid nitrogen in a large plastic beaker (weigh this beaker first as a reference). This will form noodles of yeast that should then be broken into smaller pieces. Repeat this step several times to freeze the entire pellet, and transfer to a plastic bottle that has been pre-chilled with liquid nitrogen.

8b. Alternatively use a large spatula to scoop up the pellet in large chunks and freeze them directly in liquid nitrogen contained in a plastic beaker. Use two plastic beakers, one inside the other, in case one breaks. With the spatula or in a large, chilled mortar and pestle, break the pellet into small pieces.

9. Weigh the frozen cell pellet and subtract the bottle reference weight. Store at $-80{ }^{\circ} \mathrm{C}$.

\section{Lysate preparation and affinity purification}

1. Prepare 4X Dynein Lysis Buffer with additives and leave on ice.

2. Grind the cells in batches in a coffee grinder pre-chilled with liquid nitrogen. A typical coffee grinders efficiently grinds about $50 \mathrm{~g}$ of cells. Therefore, grind in batches and pool the powder in 
a plastic beaker that is surrounded by dry ice or liquid nitrogen. When all grinding is finished, quickly transfer the powder to a glass beaker held at room temperature.

3. Add the appropriate amount of prepared $4 \mathrm{X}$ lysis buffer $(0.33 \mathrm{~mL}$ per $1 \mathrm{~g}$ cell weight) to the powder and quickly thaw in a $37^{\circ} \mathrm{C}$ water bath while stirring with a spatula. When almost thawed, transfer the beaker containing the lysate to bucket with ice, add a bit of PMSF to get rid of bubbles and continue stirring until you get a homogeneous suspension.

4. Transfer the lysate to Ti 45 centrifugation tubes, balance, and centrifuge for 2 hours at 40,000 $\mathrm{rpm}$. Note: 2 hours is a unusually long centrifugation time for lysate preparation. The reason for its inclusion is that ribosomes, a common dynein contaminant, can be efficiently removed from the lysate by extended ultracentrifugation (Andrew Carter, personal communication).

5. Carefully pool the supernatant in a chilled $250 \mathrm{~mL}$ glass bottle on ice. Take care to avoid the loose pellet at the bottom of the tube, and any lipids at the air-water interface,

Note: The lysate preparation procedure described above is applicable to many constructs. This protocol continues to describe the affinity purification of ZZ-tagged dynein. The ZZ tag binds with high affinity to $\operatorname{IgG}(3)$. When a TEV protease cleavage site is placed C-terminal of the ZZ tag, the protein can be efficiently cleaved directly off the resin by the addition of TEV protease. 
6. Estimate the volume of lysate recovered and prepare a small column packed with $\operatorname{IgG}$ Sepharose Fast Flow (GE Healthcare) at a ratio of $1 \mathrm{~mL}$ per $100 \mathrm{~g}$ of starting material.

7. Set up a peristaltic pump (all lines pre-washed with lysis buffer) to repeatedly pass (loop) the lysate over the column at $1-1.5 \mathrm{~mL} / \mathrm{min}$. Let the pump run for a total volume twice the volume of lysate. Check the column occasionally to be sure the level of liquid in the column is constant.

8. Set up the peristaltic pump to wash the column with Wash Buffer at $1-1.5 \mathrm{~mL} / \mathrm{min}$ for approximately 100 column volumes. Monitor the absorbance at $280 \mathrm{~nm}$ of the flow through to establish the endpoint of washing (i.e. the $\mathrm{A}_{280}$ has flatlined).

9. Wash the column at $1-1.5 \mathrm{~mL} / \mathrm{min}$ with $\sim 10$ column volumes TEV Buffer. When the buffer meniscus just touches the top of the resin, stop the flow and use a pipette and $10 \mathrm{~mL}$ TEV buffer to transfer the resin to a chilled $15 \mathrm{~mL}$ falcon tube.

10. Add TEV protease at this point at a ratio of $\sim 1 \mu \mathrm{L}$ per $200-400 \mu \mathrm{L}$ resin. Mix by capping and gently inverting. Note: TEV can be purchased from a number of vendors, or easily purified in lab. Test the activity of lab purified protein to determine the optimum amount to use, as the amount indicated above refers to a specific prep (SRP lab JH prep '09).

11. Use additional rinses with $1 \mathrm{~mL}$ TEV buffer to transfer the remaining resin to the Falcon tube. Cap and incubate overnight at $4^{\circ} \mathrm{C}$. It is extremely important that the falcon tube is 
completely full before capping. VERY IMPORTANT: continue adding TEV buffer until the buffer meniscus exceeds the top of the tube. Gently add the cap (some buffer should overflow) and tighten. Wrap the top with parafilm and rotate about the long axis of the tube overnight at $4^{\circ} \mathrm{C}$. Be sure to look for large air bubbles in the tube. If any are present, remove them and refill the tube. The presence of large air bubbles will cause dynein to precipitate during the TEV cleavage reaction.

12. Centrifuge the tube at $5000 \mathrm{rpm}$ for 10 minutes at $4^{\circ} \mathrm{C}$.

13. Transfer the supernatant to a fresh, chilled Falcon tube by passing it through a small, chilled chromatography or filter column. This is intended to remove any residual resin before concentrating the sample.

14. Prepare an Ultracell 100K MWCO concentrating column by centrifuging at $4000 \mathrm{rpm}$ for $\sim 5$ minutes at $4^{\circ} \mathrm{C}$ with $4 \mathrm{~mL}$ of TEV buffer. This step washes the membrane to remove any preservatives.

15. Remove all TEV buffer and add $4 \mathrm{~mL}$ of the TEV cleavage reaction to each concentrating column. Alternatively, a larger $25 \mathrm{~mL}$ concentrating column can be used. Centrifuge at $4000 \mathrm{rpm}$ in a swinging bucket rotor at $4{ }^{\circ} \mathrm{C}$. Note: Start with an initial 5 minute spin and judge how fast the solution is passing over the filter. Gently mix the solution after each spin, being sure to rinse the membranes while avoiding the formation of bubbles. Decrease the centrifugation times as the 
volume approaches the desired value. For GFP tagged proteins a convenient way to judge the concentration is to look at the color of the solution. At the point that the solution is visibly green, it has approached $\sim 1 \mathrm{mg} / \mathrm{mL}$.

16. Pool the concentrated protein and spin through a chilled filter column to remove any remaining resin.

17. The protein can be flash frozen at this point or applied directly to an equilibrated gel filtration column to remove TEV and contaminating proteins. Concentrated dynein solutions can tolerated a single freeze-thaw cycle.

\section{Gel filtration}

Dynein (monomer or dimer) can be efficiently separated from contaminants, including rTEV, on a Superdex 200 or Superose 6 columns. For large preps, then entire concentrated IgG eluate can be loaded on $120 \mathrm{~mL}$ columns. In Gel Filtration Buffer (see recipe), monomeric dynein elutes at $\sim 55 \mathrm{~mL}$, and dimeric dynein elutes a $49 \mathrm{~mL}$ on a $120 \mathrm{~mL}$ Superdex 200 columns (void $=40 \mathrm{~mL} ; \mathrm{V}_{\mathrm{e}} / \mathrm{V}_{\mathrm{o}}$ of 1.375 for monomer and 1.225 for dimer). Initially it is worthwhile to analyze fractions by SDS-PAGE + Coomassie Blue staining. Peak fractions can be pooled and concentrated as described above.

As an alternative to running the entire IgG eluate on a large gel filtration column, one can run aliquots (50-100 $\mu \mathrm{L}$ at $1-2 \mathrm{mg} / \mathrm{mL})$ on small columns connected to micro-scale FPLC machines An example is the Akta Micro (GE Healthcare) which utilizes $2.4 \mathrm{~mL}$ columns (Figure 
A2.3). The peak fractions, which have undergone buffer exchange on the column into a suitable cryo-EM buffer, are typically of high enough concentration to use directly in EM grid preparation.

\section{Microtubule polymerization}

This is a basic protocol for polymerizing MTs in in vitro. The MTs can be used for a variety of assays, including binding assays, single molecule studies, and EM grid preparation. Tubulin, in the form of alpha-beta heterodimers, can be isolated from calf brain, or purchased from Cytoskeleton, Inc. Purchased tubulin is ultra-pure and is convenient for approaches that do not require large amounts, such as EM.

1. Turn on a heat block and be sure it is stable at $37^{\circ} \mathrm{C}$. MT polymerization is temperature dependent, so be sure to trust a thermometer, not the reading on the machine.

2. Prepare $1 \mathrm{X}$ BRB $80+1 \mathrm{mM}$ DTT and $3 \mathrm{mM}$ GTP. Prepare from $5 \mathrm{X}$ BRB80.

3. Prepare $2 \mathrm{mM}$ taxol (Sigma) from a $10 \mathrm{mM}$ stock in DMSO, using DMSO to dilute.

4. Prepare the polymerization mixture. Note: if using Cytoskeleton, Inc. tubulin, resuspend the lyophilized protein powder in 1X BRB80 + $1 \mathrm{mM}$ DTT (omit GTP). Spin the resuspended protein at $50 \mathrm{~K}$ in a TLA100 rotor at $4 \mathrm{C}$ for 30 minutes to remove and aggregates. Make $\sim 22 \mu \mathrm{L}$ aliquots and snap freeze in LN2 and store @ -80 C. Remove an aliquot for polymerization and thaw quickly with your hands - transfer to ice just as the last bit of ice is thawing. Mix together $20 \mu \mathrm{L}$ thawed tubulin and $10 \mu \mathrm{L}$ of $1 \mathrm{xBRB} 80$ containing $1 \mathrm{mM}$ DTT and $3 \mathrm{mM}$ GTP. 
5. Gently mix the two solutions and transfer immediately to $37^{\circ} \mathrm{C}$ heat block filled with water. This will give a tubulin concentration of approximately $6.67 \mathrm{mg} / \mathrm{mL}$ or $\sim 67 \mu \mathrm{M}$.

6. Incubate at $37^{\circ} \mathrm{C}$ for 45 minutes with additions of $2 \mathrm{mM}$ taxol (aka paclitaxel, SigmaAldrich). At 15 minutes add $0.5 \mu \mathrm{L}$. At 30 minutes add $0.5 \mu \mathrm{L}$. At 45 minutes add $1.0 \mu \mathrm{L}$.

7. Incubate for another hour at $37 \mathrm{C}$. At this point the MTs are stable and can be stored at RT for extended periods of time (up to several days, though for grid preparation it is best to use immediately).

\section{Dynein-MT cryo-EM grid preparation}

Preparing cryo-EM grids with MTs + bound motor proteins is not overly difficult, but it does require some planning and the appropriate timing of steps. When you start plunging grids you will have successfully dialyzed your protein, polymerized MTs, charged your grids, and prepared the Vitrobot (FEI) such that they are all ready simultaneously. Plan accordingly!

Motor proteins bound to MTs can be prepared in several ways. The MTs and motor proteins can be incubated and then centrifuged through a sucrose gradient to isolate on the complex. Alternatively, the complex can be formed directly on the grid by sequentially applying MTs and motor protein. This protocol describes how to form the complex directly on an EM grid (Figure A2.3). 
1. The previous day be sure that one LN2 dewar is empty and placed upside down to completely dry out

2. Prepare microtubules as described in Microtubule preparation (above)

3. Dialyze your motor protein into a suitable buffer. This is protein dependent, but for dynein work it is advisable to use low salt (salt decreases binding, which is governed primarily by electrostatic interactions), and low amounts of glyerol (high levels decrease contrast in cryoEM). For example, dynein constructs (monomer, dimer, SRS-MTBD) are dialyzed into Gel Filtration Buffer (recipe below) for at least 4 hours at $4^{\circ} \mathrm{C}$.

4. Centrifuge the proteins for 30 minutes at max speed in a microcentrifuge @ $4 \mathrm{C}$.

5. Dilute MTs appropriately. For cryo-EM, where the goal is to have several MTs per hole, a dilution to $5 \mu \mathrm{M}$ (1:12 from standard polymerization protocol) works well. Dilute into dialysis buffer $+100 \mathrm{uM}$ taxol that has been filtered through a $0.2 \mathrm{uM}$ syringe filter. Use tips that have had the ends cut off with a razor blade and pipette and swirl gently to resuspend. Here it is a balance between just enough resuspension, which reduces large clumps of MTs that make data collection impossible, and too much, which causes the MTs to shear and fragment.

6. Glow discharge the grids to encourage even spreading of the solution. It is advisable to use manufactured grids for their superior performance. C-flat from Protochips works well for grids containing MTs. Be sure to chose a hole size and spacing that works for the magnification you 
intend to work at. Briefly glow discharge (15-20 seconds) @ $30 \mathrm{~mA}$. The time and power used for glow discharge is machine and season dependent. It is advisable to screen conditions with MTs before potentially wasting valuable motor protein.

7. Prepare the ethane pot and Vitrobot (FEI) for plunging. Settings will vary for each protein, buffer, grid, etc., so anticipate a lot of screening to find the right conditions. It is advisable to talk to current lab member to get a sense of what constitutes current favorable settings. Constant settings are $22{ }^{\circ} \mathrm{C}, 100 \%$ humidity.

8. Allow the dialyzed motor protein solution to warm to RT.

9. Apply $4 \mu \mathrm{L}$ of diluted MTs (with a P20 tip that has had the end cut off) to a grid held in Vitrobot tweezers which has been positioned outside of the Vitrobot. Allow the MTs to absorb for 30 seconds. Note: if the solution passes through to the other side of the grid it has been glow discharged too harshly and will not give reproducibly good ice.

10. Blot gently from the side with a the edge of a torn piece Whatman \#1 filter paper

11. Apply $4 \mu \mathrm{L}$ of motor protein solution. Depending on the construct this can be done once for up to one minute. Again, if the solution passes through to the other side, the ice is unlikely to be of good quality. For maximal decoration, blot the protein as above (step 11), and repeat the application/blotting or protein solution a total of 3-4 times. After the final addition proceed to step 12. 
12. Mount the tweezers in the Vitrobot and proceed to blotting and plunging. Store grids in liquid nitrogen for at least one day to remove contaminating ethane.

\section{Buffers}

$\underline{2 \mathrm{xYP}+1 \% \text { glucose }}$

$20 \mathrm{~g} / \mathrm{L}$ Yeast extract

$40 \mathrm{~g} / \mathrm{L}$ Peptone

$10 \mathrm{~g} / \mathrm{L}$ glucose

$\underline{40 \% \text { galactose }}$

$400 \mathrm{~g} / \mathrm{L}$ galactose (weigh the powder and transfer to large beaker, warm the solution up to $\sim 60^{\circ} \mathrm{C}$ or until everything is dissolved, aliquot and filter sterilize).

5x Lysis Buffer

150 mM HEPES, $\mathrm{pH} 7.4$

$250 \mathrm{mM}$ KOAc

$10 \mathrm{mM} \mathrm{MgOAc}$

5 mM EGTA

$50 \%$ glycerol

4x Lysis Buffer (use 5x Lysis Buffer to make)

120 mM HEPES, $\mathrm{pH} 7.4$

$200 \mathrm{mM}$ KOAc

$8 \mathrm{mM} \mathrm{MgOAc}$

4 mM EGTA

$40 \%$ glycerol

$4 \mathrm{mM}$ DTT, freshly added

$0.4 \mathrm{mM}$ Mg-ATP, freshly added

$0.2 \%$ Triton X-100

$4 \mathrm{mM}$ PMSF, freshly added

$4 \mu \mathrm{g} / \mathrm{mL}$ each of aprotinin, leupeptin, antipain, pepstatin

Wash Buffer (equivalent to $1 \mathrm{X}$ Lysis Buffer $+250 \mathrm{mM} \mathrm{KCl}$, use $4 \mathrm{X}$ Lysis Buffer with additives to make)

40 mM HEPES, $\mathrm{pH} 7.4$

$50 \mathrm{mM} \mathrm{KOAc}$

$2 \mathrm{mM} \mathrm{MgOAc}$ 
$1 \mathrm{mM}$ EGTA

$10 \%$ Glycerol

$1 \mathrm{mM}$ DTT, freshly added

$0.1 \mathrm{mM}$ Mg-ATP, freshly added

$0.05 \%$ Triton X-100

$1 \mathrm{mM}$ PMSF, freshly added

$1 \mu \mathrm{g} / \mathrm{mL}$ each of aprotinin, leupeptin, antipain, pepstatin

$250 \mathrm{mM} \mathrm{KCl}$

TEV Buffer

$50 \mathrm{mM}$ Tris- $\mathrm{HCl}, \mathrm{pH} 8.0$

$150 \mathrm{mM} \mathrm{KOAc}$

$2 \mathrm{mM} \mathrm{MgOAc}$

$1 \mathrm{mM}$ EGTA

$10 \%$ glycerol

$1 \mathrm{mM}$ DTT

$1 \mathrm{mM}$ PMSF

$1 \mu \mathrm{g} / \mathrm{mL}$ each of aprotinin, leupeptin, antipain, pepstatin

Gel Filtration Buffer (also referred to as Cryo Buffer)

$50 \mathrm{mM}$ Tris- $\mathrm{HCl}, \mathrm{pH} 8.0$

$150 \mathrm{mM} \mathrm{KOAc}$

$2 \mathrm{mM} \mathrm{MgOAc}$

$1 \mathrm{mM}$ EGTA

$5 \%$ glycerol

$1 \mathrm{mM}$ DTT

$\underline{\text { 5X BRB80 }}$

400 mM K-PIPES, pH 6.8

$5 \mathrm{mM} \mathrm{MgCl}_{2}$

5 mM EGTA. 


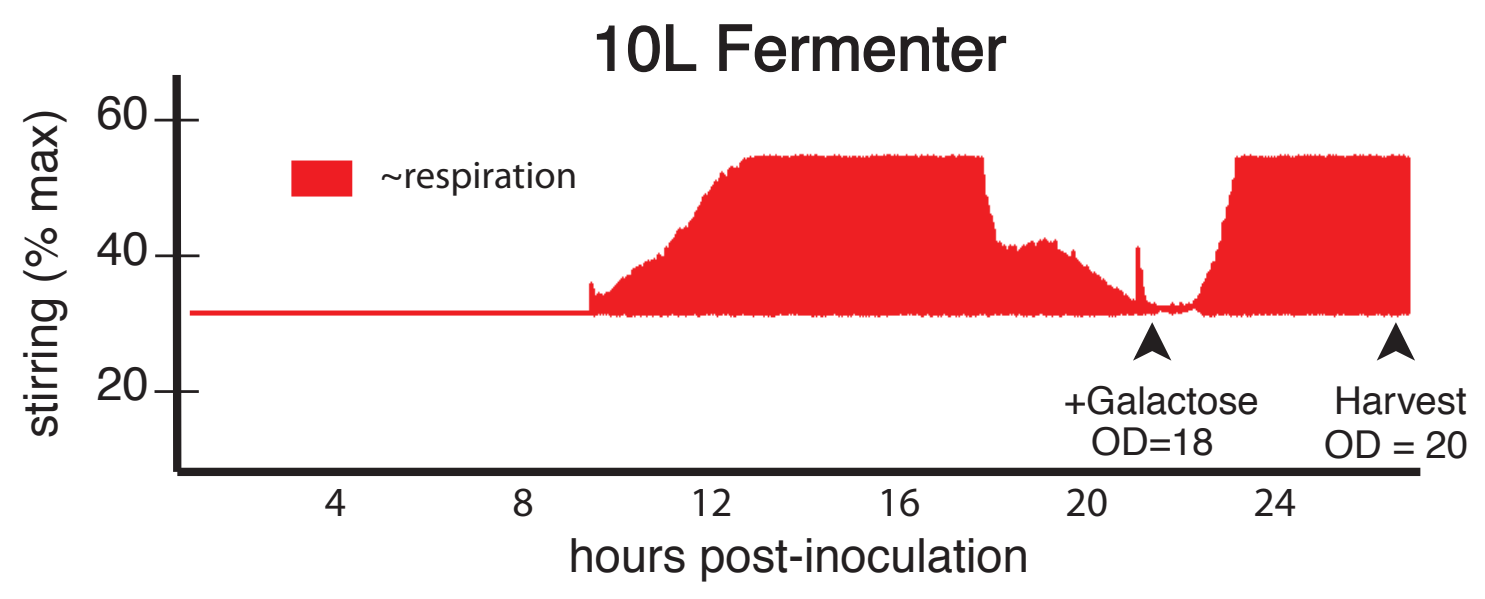

Figure A2.1. Response of yeast harboring Gal-inducible dynein minidimer to galactose following glucose depletion. In this graph the respiration of a yeast culture is monitored by the percent maximal stirring of the machine needed to maintain a constant dissolved oxygen level. Nearing 20 hours post-inoculation, the culture approaches a stagnation in growth, at which point galactose was added. Following a brief lag, the culture begins a phase of increased oxygen demand. Total yield from this batch of cells (475 g) was approximately $4 \mathrm{mg}$. 


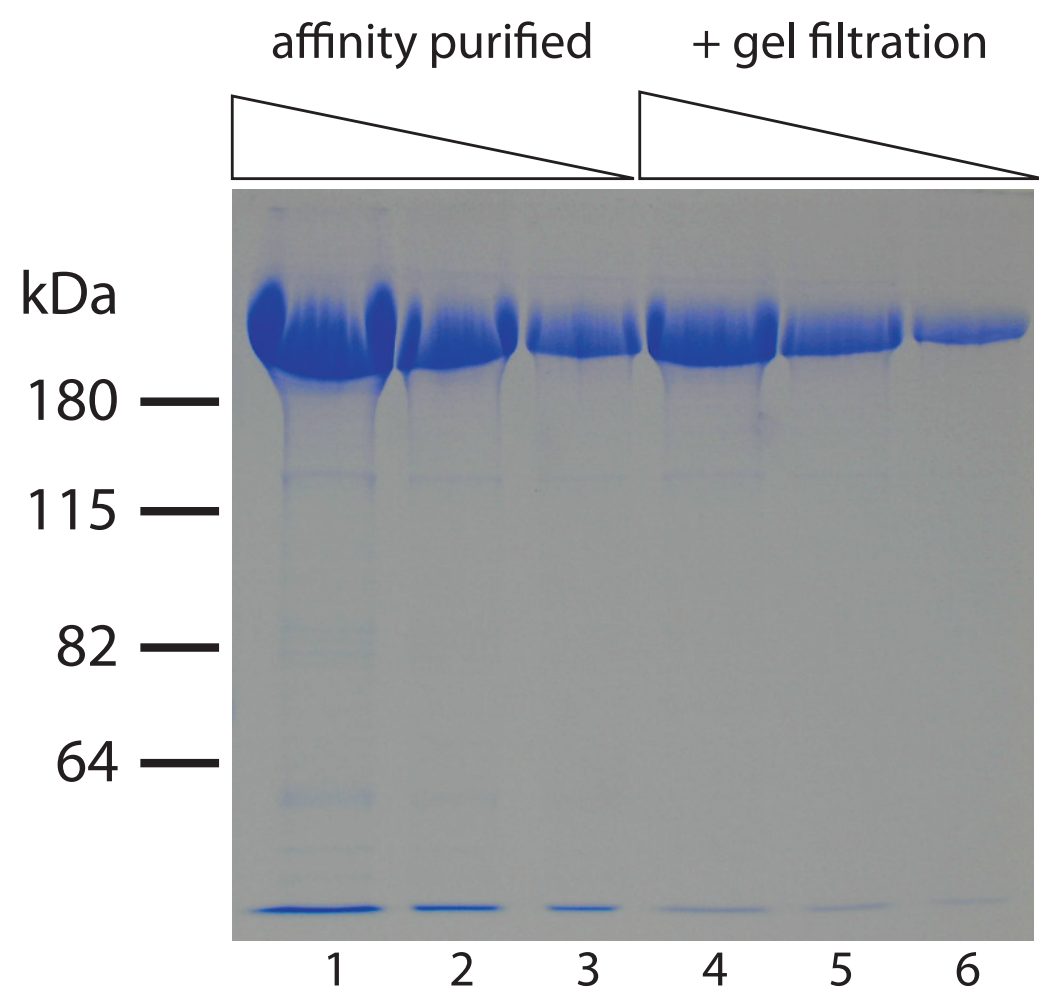

Figure A2.2. Large scale purification of dynein minidimer. $20 \mathrm{~L}$ of yeast culture were grown and induced as described in this appendix. The total cell wet weight was 500 g. Serial dilutions of the concentrated IgG-agarose eluate (lanes 1-3), and concentration peak fractions from gel filtration chromatography (S200 16/60, lanes 4-6) were separated on a 4-12\% SDS-PAGE gel and visualized by Coomassie Brilliant Blue (Sigma) staining. The concentration of the sample post-gel filtration is $\sim 2 \mathrm{mg} / \mathrm{mL}$ (as compared to a BSA standard, not shown). The final total yield is $\sim 2$ mg. 


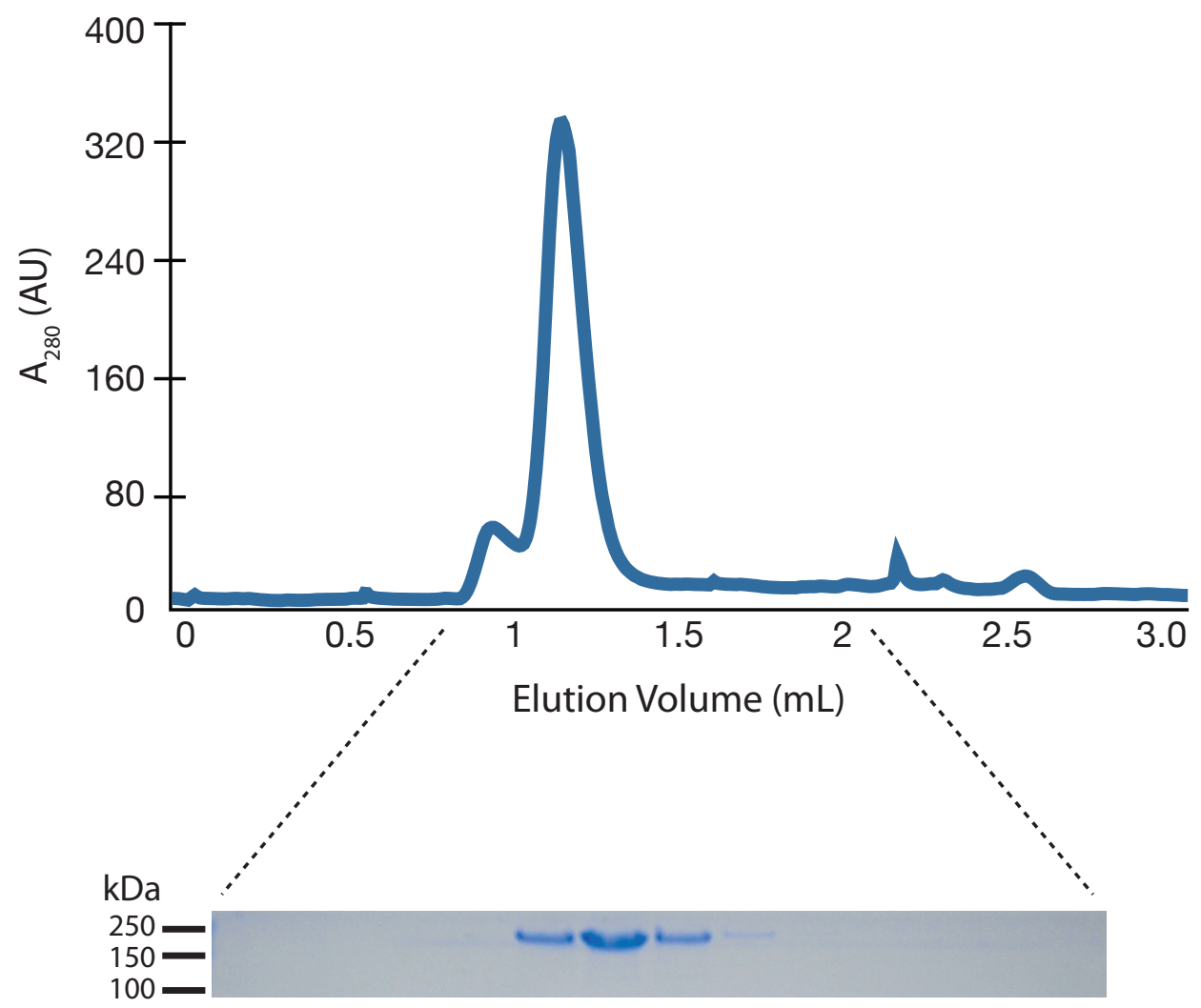

Figure A2.3. Gel filtration chromatography of IgG-agarose affinity purified ZZtagged dynein minidimer. $100 \mu \mathrm{L}$ of $\sim 2 \mathrm{mg} / \mathrm{mL}$ minidimer was run on a $2.4 \mathrm{~mL}$ Superose 6 connected an Akta Micro FPLC (GE Healthcare). The three peak fractions were pooled an applied directly to EM grids pre-absorbed with MTs. 

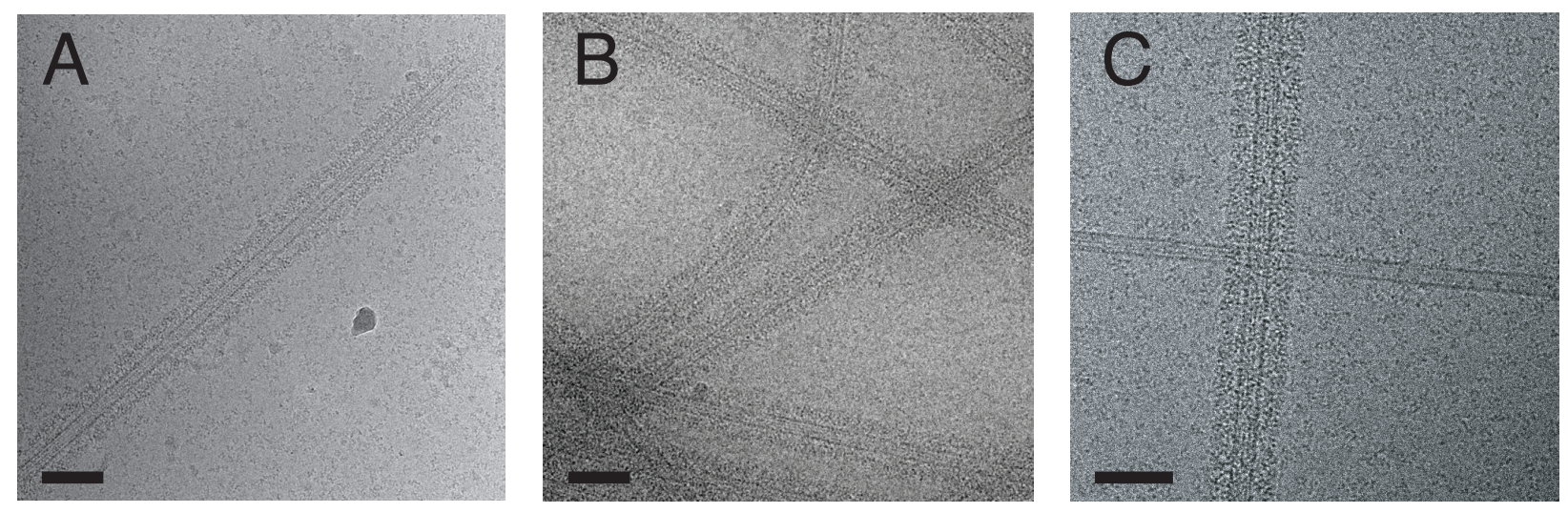

Figure A2.4. Highly decorated microtubules imaged under cryogenic conditions. (A) A microtubule highly decorated with monomeric dynein. (B) A field of microtubules uniformly decorated with dynein minidimer. (B) An occasionally observed phenomenon, whereby highly decorated and undecorated MTs are present in the same micrograph. We have also observed this in negatively stained samples. Scale bars represent $100 \mathrm{~nm}$. Note the free dynein in MT devoid regions due to the omission of buffer washes. 


\section{References}

1. S. L. Reck-Peterson et al., Single-molecule analysis of dynein processivity and stepping behavior, Cell 126, 335-348 (2006).

2. M. Werner-Washburne et al., Stationary phase in the yeast Saccharomyces cerevisiae, Microbiol. Rev. 57, 383-401 (1993).

3. C. Chen et al., Immobilized protein ZZ, an affinity tool for immunoglobulin isolation and immunological experimentation, Biotechnol Appl Biochem 45, 87-92 (2006). 